\title{
The role of microRNAs in the p53 tumor suppressor pathway
}

\section{Dissertation}

\author{
for the award of the degree \\ “Doctor of Philosophy” (Ph.D.) \\ Division of Mathematics and Natural Sciences \\ of the Georg-August-Universität Göttingen
}

\author{
submitted by \\ Xin Zhang \\ Born in Wuhan, China
}

Göttingen, May 2010 


\section{Thesis supervisor:}

Prof. Dr. Matthias Dobbelstein

Department of Molecular Oncology, Georg-August-Universität Göttingen

\section{Members of my thesis committee:}

Prof. Dr. Matthias Dobbelstein $\left(1^{\text {st }}\right.$ Referee $)$

Department of Molecular Oncology, Georg-August-Universität Göttingen

Prof. Dr. Thomas Pieler

Department of Developmental Biochemistry, Georg-August-Universität Göttingen

Prof. Dr. Jürgen Wienands

Department of Cellular and Molecular Immunology, Georg-August-Universität Göttingen

\section{Additional Reviewers:}

Prof. Dr. Andreas Wodarz

Department of Stem Cell Biology, Georg-August-Universität Göttingen

Prof. Dr. Heidi Hahn

Department of Molecular Developmental Genetics, Georg-August-Universität Göttingen

Prof. Dr. Felix H. Brembeck

Department of Hematology and Oncology, Georg-August-Universität Göttingen

Date of the oral examination: June $22^{\text {nd }}, 2010$ 


\section{Affidavit}

I hereby declare that my doctoral thesis entitled "The role of microRNAs in the p53 tumor suppressor pathway" has been written independently and with no other sources and aids than quoted.

Xin Zhang

Göttingen, May 2010 


\section{Table of Contents}

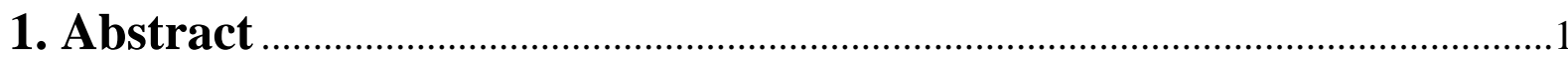

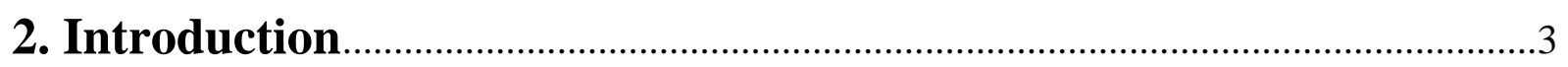

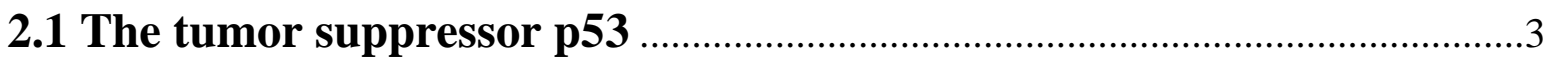

2.1.1 p53 structure .............................................................................................

2.1.2 Cellular functions of $\mathrm{p} 53$.................................................................................

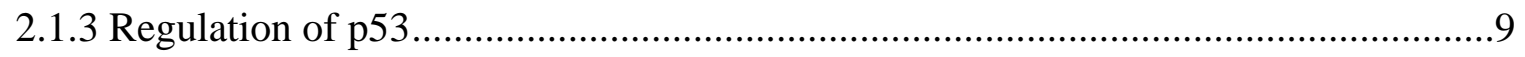

2.2 microRNAs, novel gene regulatory molecules...........................................14

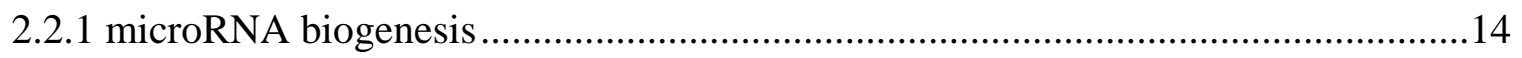

2.2.2 Mechanisms of miRNA-directed gene regulation ...................................................19

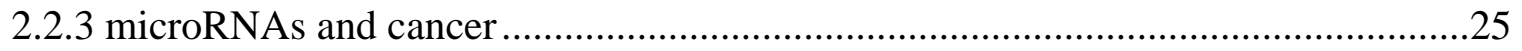

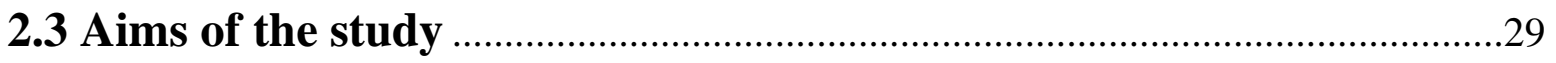

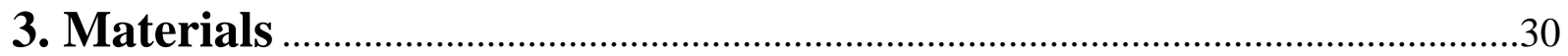

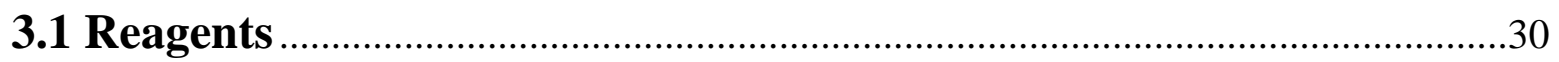

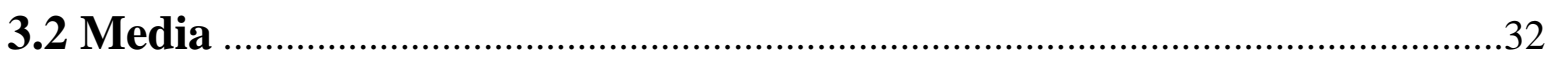

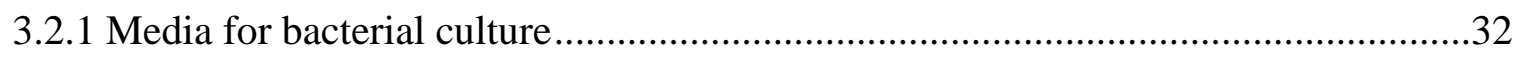

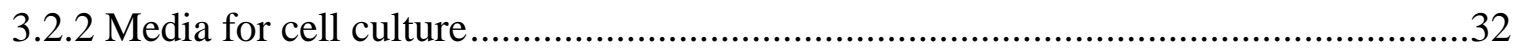

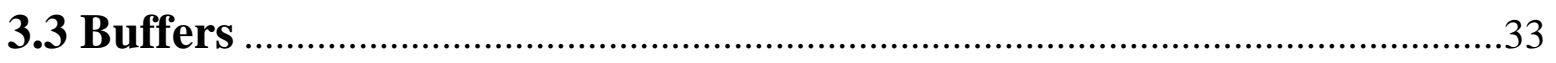

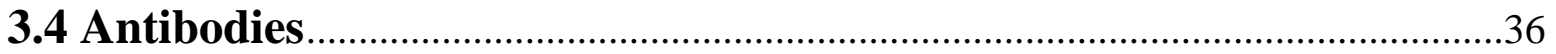

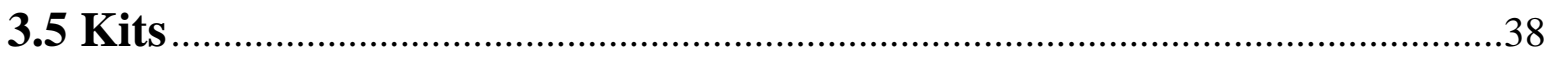

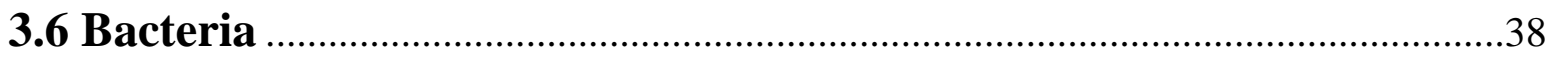

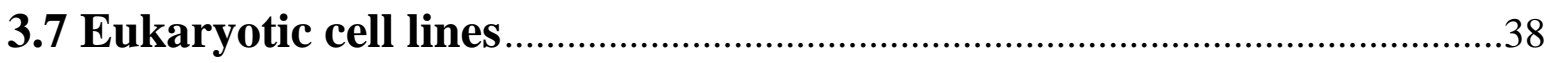

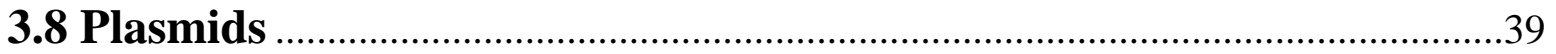




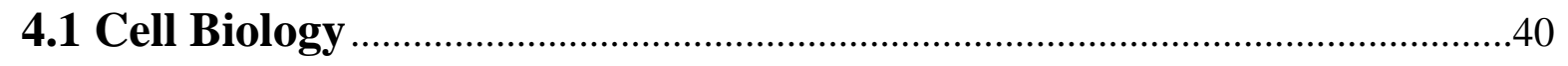

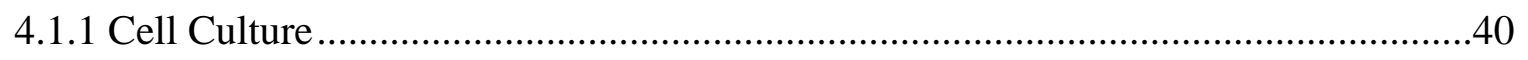

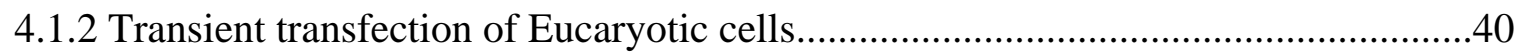

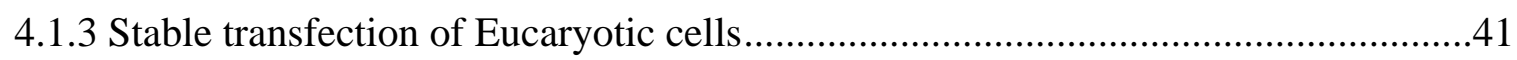

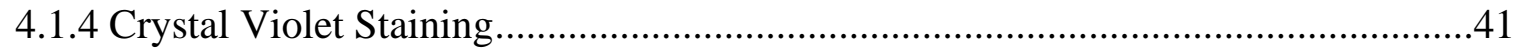

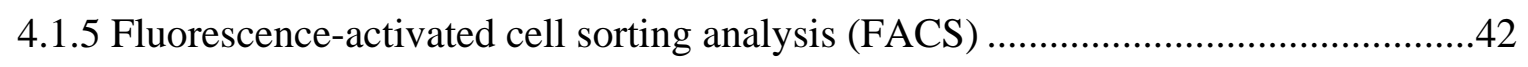

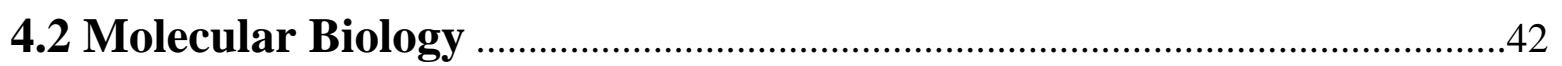

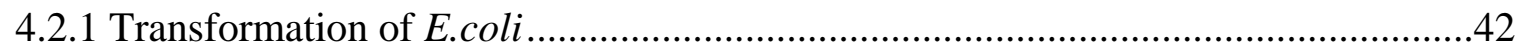

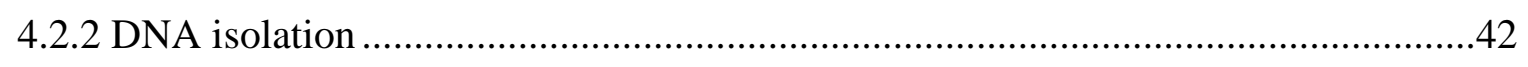

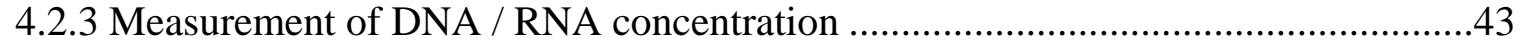

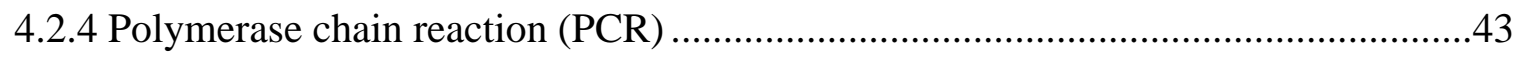

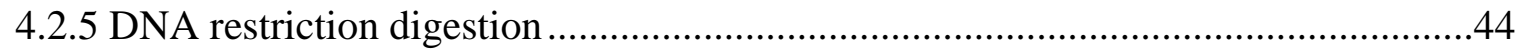

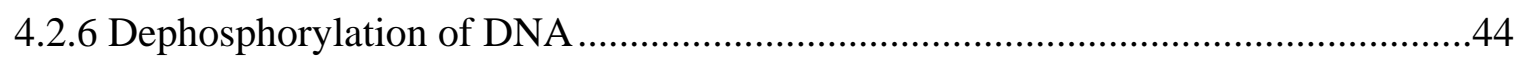

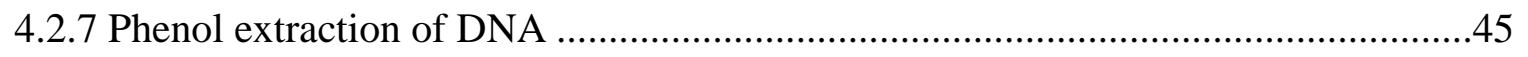

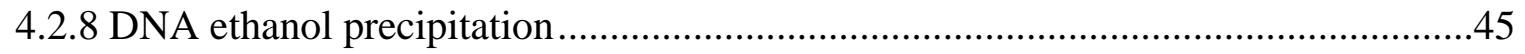

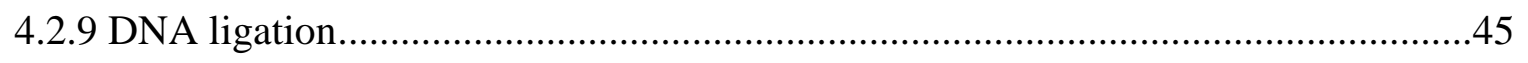

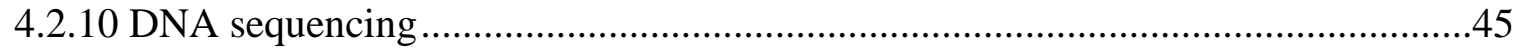

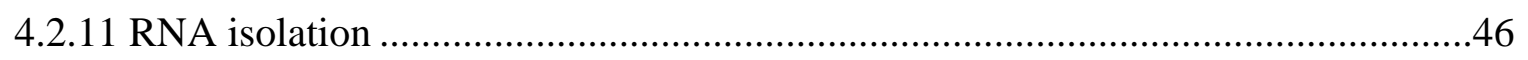

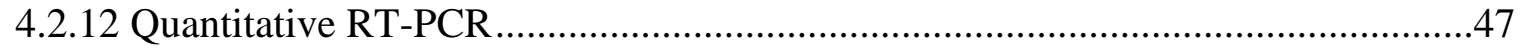

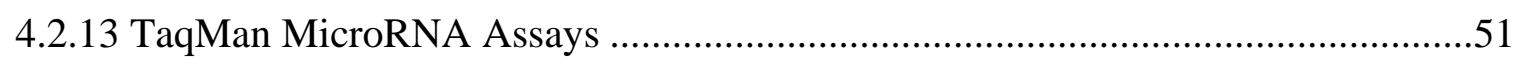

4.3 Biochemistry and Immunological methods …………………………….....53

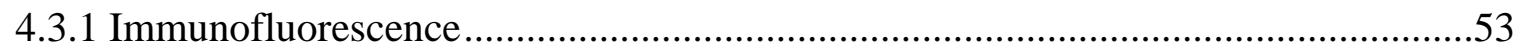

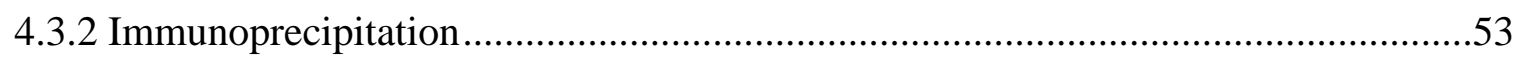

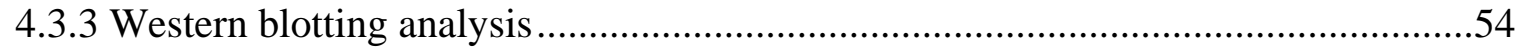

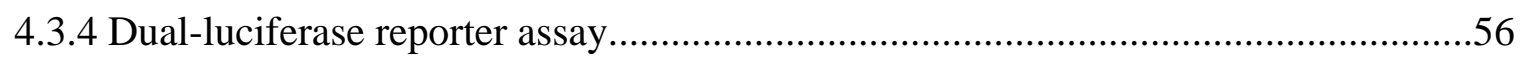

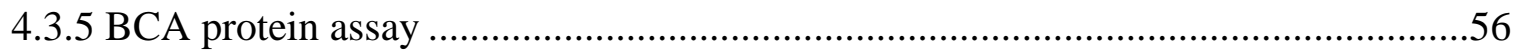

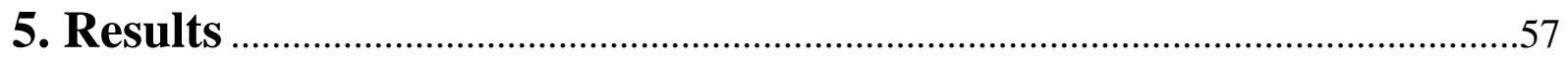




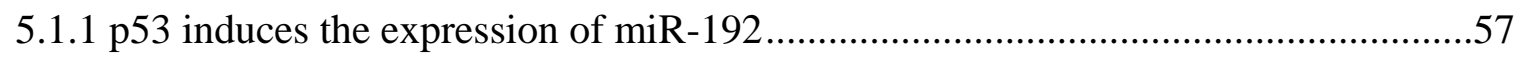

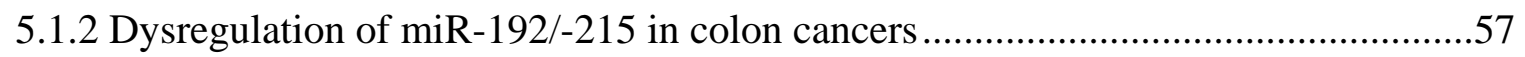

5.1.3 miR-192 suppresses cell clone formation in a partially p53-independent manner...59

5.1.4 microRNA-192/-215 provides a positive feedback to the p53 pathway...................61

5.1.5 microRNA-192/-215 is capable of inducing cell cycle arrest..................................63

5.1.6 Knockdown of miR-192 sensitizes A549 cells to 5-fluorouracil.............................63

\subsection{Identifying p53 regulatory microRNA-22 ……………………………......65}

5.2.1 Immunofluorescence screening for p53-regulatory microRNAs............................65

5.2.2 miR-22 down-regulates p53 levels and activities ................................................67

5.2.3 miR-22 does not directly target $p 53$ and $p 21$ mRNAs..........................................68

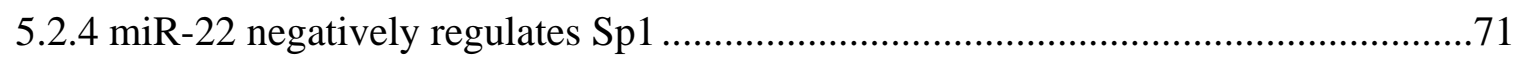

5.2.5 miR-22 suppresses p53 activation by down-regulating Chk2 …...........................72

5.2.6 miR-22 induces DNA damage and is capable of inducing G2/M arrest...................73

5.2.7 miR-22 suppresses cell clone formation .......................................................... 73

5.3 Characterization of p53 modifications induced by miR-101 ……...........76

5.3.1 miR-101 increases p53 levels and accumulates modified p53 …...........................76

5.3.2 p53-modification induced by miR-101 is dependent on Mdm2 …........................76

5.3.3 miR-101 suppresses the histone methyltransferase EZH2 _................................... 78

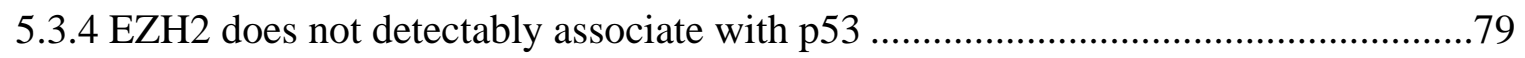

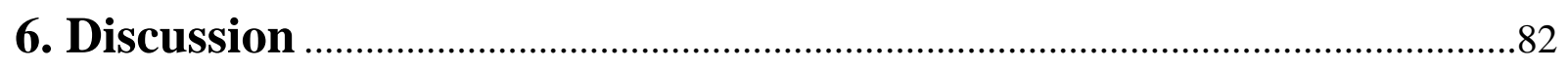

6.1 miR-192/215 act as p53 effectors and regulators ………………………...82

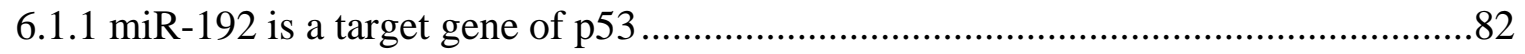

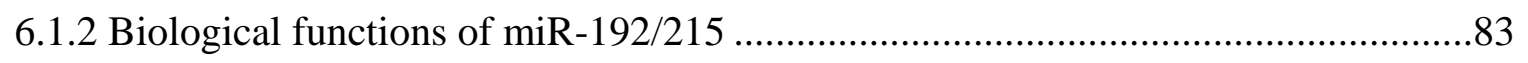

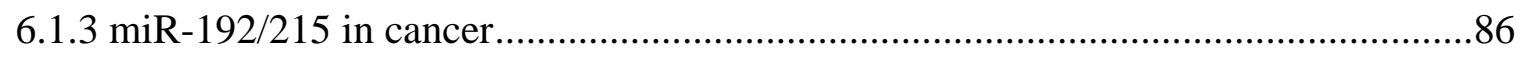

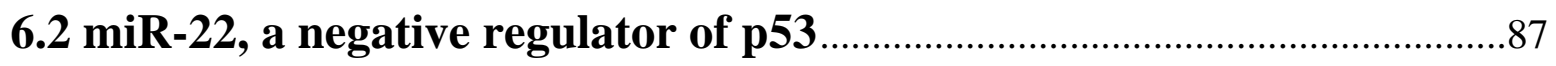

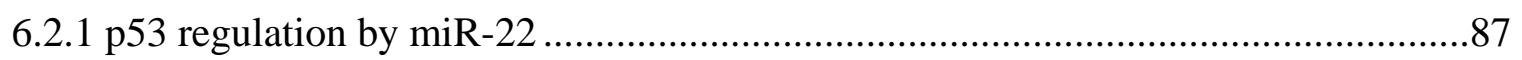

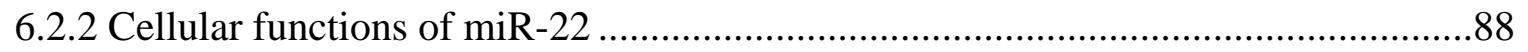




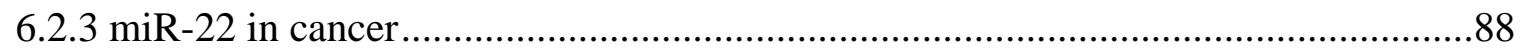

6.2.3 Does c-Myc increases p53 levels by repressing miR-22 …..................................89

6.3 Post-translational modification of p53 by miR-101 and its biological

functions

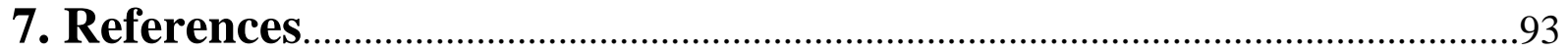

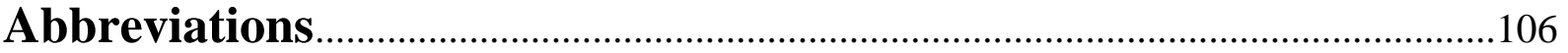

Curriculum Vitae

Acknowledgements .........................................................................................111 


\section{Abstract}

MicroRNAs (miRNAs) are a class of small, non-coding, double-stranded RNA molecules. In metazoan cells, miRNAs recognize their target mRNAs by imperfectly complementary binding, leading to translational repression or mRNA decay. Because of the importance of miRNAs in regulating cell differentiation, proliferation and apoptosis, miRNAs are implicated to be involved in human tumorigenesis. In line with this, decreased levels of global miRNA expression were observed in cancer, and increased number of specific miRNAs has been identified to function as oncogenes or tumor suppressors.

As a transcription factor, p53 regulates many downstream target genes, through which it controls cell fates to maintain genomic integrity. Here, we reported that several miRNA genes (miR-192/194/215) were also p53-responsive. In cancer cells with wild type p53, they were up-regulated in response to the treatment of Nutlin-3 (a small molecule which inhibits Mdm2 and p53 interaction, resulting in p53 stabilization) or DNA damaging agents. All three miRNAs were found to be down-regulated in colon cancers relative to normal colon tissue, suggesting that they might have tumor suppressive activities. To support this, miR-192 suppressed clonogenic survival. Furthermore, miR-192/215 induced cell cycle arrest, which was at least partially dependent on p53 and p21. Overexpression of miR-191/215 in several cancer cell lines induced p53 and its target gene product p21, providing a positive feedback to the p53 pathway. Knockdown of endogenous miR-192 in A549 cells attenuated p53 and p21 activation in response to 5-fluorouracil treatment. Hence, miR-192 and miR-215 act as effectors as well as regulators of p53.

Using highly parallel transfection of microRNA expression clones and subsequent high-content immunofluorescence microscopy, we further identified two miRNAs (miR-22 and miR-101) that can regulate $\mathrm{p} 53$.

Overexpression of ectopic miR-22 suppressed p53 and p21 levels. p53 phosphorylation (Ser15 and 20) and acetylation (Lys382), in response to DNA damaging agent, were profoundly impaired by miR-22. Both Chk2 (one protein kinase which can target p53) and Sp1 (one p53 cofactor) were found to be down-regulated by miR-22. Rather than an oncogenic miRNA, miR-22 suppressed clonogenic survival and triggered G2/M arrest. 
In contrast to miR-22 as a negative regulator of p53, miR-101 induced p53 and its target gene products p21 and Mdm2. More strikingly, miR-101 also accumulated modified p53, which was reminiscent of mono-ubiquitinated p53. Antagonizing Mdm2-p53 interaction by Nutlin-3 or knockdown of Mdm2 abolished miR-101-induced p53 modification. Knockdown of other p53 modifiers (such as p300, CBP, HAUSP and its homologue USP47) respectively, did not phenocopy miR-101-induced p53 modification. Interestingly, knockdown of EZH2 (one target of miR-101) by one siRNA, also induced the same pattern of modified p53, although it needs to be further confirmed.

Taken together, our results indicated that miRNAs constitute part of the p53 tumor suppressor network and contribute to its biological activities. These miRNAs might be novel therapeutic targets for diagnosis and treatment of human cancers. 


\section{Introduction}

\subsection{The tumor suppressor p53}

In the year 2009 , the $30^{\text {th }}$ anniversary of the discovery of p53 was celebrated. p53 has been called "the guardian of the genome" because of its essential role in maintaining genomic integrity (Lane 1992). In response to a variety of stresses including DNA damage, p53 is stabilized and activated. Once activated, p53 can trigger cell cycle arrest, senescence or apoptosis, therefore eliminating cell-transformation potential. Thus, loss of p53 function might be one mechanism employed by transforming cells. Indeed, p53 mutations have been observed in approximately $50 \%$ of malignant tumors. More direct evidences came from a knockout mice model, where p53 null mice is developmentally normal but more susceptible to spontaneous tumors (Donehower, Harvey et al. 1992). Currently, the functions of p53 and its regulation are still under intensive investigation, which may contribute to cancer therapy in the future.

\subsection{1 p53 structure}

The human p53 protein consists of 393 amino acids. According to its structure and function, p53 protein can be divided into five domains.

Two transactivation domains (TAD 1, residues 20-40; TAD 2, residues 40-60) are located in the N-terminus. Both interact with the basal transcriptional machinery and are required for full transactivation ability.

The proline-rich domain (residues 60-90) contains five repeats of the motif "PXXP". This region has been reported to be involved in the regulation of p53-mediated apoptosis (Venot, Maratrat et al. 1998).

The sequence-specific DNA-binding domain (DBD) is frequently referred to as "the core" because of its protease resistance (Bargonetti, Manfredi et al. 1993; Pavletich, Chambers et al. 1993). It exhibits the highest homology both between p53 proteins from different species and between the different p53 family members, p63 and p73. This domain binds to a specific sequence within the target gene promoters, called p53 response elements (REs). p53 targets contain at least two spaced REs. The defined consensus sequence of RE is RRRCA/TT/AGYYY (R, purine; Y, pyrimidine). Interestingly, 97\% of the tumor-associated 
p53 mutations are mapped in the DNA-binding domain, resulting in its sequence binding activity. It suggests a vital role of the DNA-binding domain in the tumor suppressor function of p53. These mutations can be classified into two groups (Levine 1997). Mutations in amino acid residues such as R248 and R273, the two most frequently observed mutations in human cancers, directly cause DNA binding defects, resulting in the loss of p53 transactivation ability. The second group of mutations, for example R175, G245, R249 and R282, alter the conformation of $\mathrm{p} 53$.

The tetramerization domain (TET, residues 325-356) is required for the tetramer formation (as dimmer of dimers) of the p53 proteins.

The lysine-rich basic C-terminal domain (residues 363-393) has non-specific DNA binding ability and is implicated as a negative regulator of p53 sequence-specific DNA-binding. The C-terminal domain is highly post-translationally modified (such as phosphorylation, acetylation, ubiquitination, methylation, neddylation and sumoylation). Many of such modifications (such as phosphorylation and acetylation) are stress-inducible and contribute to p53 sequence-specific DNA-binding.

\subsubsection{Cellular functions of p53}

Under normal circumstances, p53 is kept at low levels due to its rapid turnover. In response to a broad range of stresses, p53 is stabilized mainly by inhibiting Mdm2, a negative regulator of and ubiquitin ligase for p53. Upon p53 activation, it regulates diverse cellular processes including cell cycle arrest, senescence, apoptosis, DNA repair, metabolism, development and aging, far more than our previous expectations. It can be anticipated that more novel functions of p53 will emerge. Although p53 can function in a transcription-independent manner, the main functions of p53 are attributed to its transcriptional activity. As a transcription factor, p53 regulates its target genes through transcriptional activation or repression. Gene expression microarrays have revealed that p53 regulates a large repertoire of genes, not limited to those involved in cell cycle arrest and apoptosis (Vousden and Prives 2009). Many other genes associated with cellular processes such as DNA repair, cell adhesion, cell mobility, angiogenesis, metabolism, and autophagy are also affected by p53 activity. 


\section{Cell cycle arrest}

Although diverse functions of p53 have been discovered, intensive attentions focus on p53 function in tumor suppression by both blocking cell cycle progression and inducing apoptosis. Although reversible cell cycle arrest may be not the safest option for p53 to prevent cancer development, it may be the most economic. Under low and transient stress, p53 arrests cells before they initiate replicative DNA synthesis (G1 arrest) and/or begin mitosis (G2 arrest), allowing optimal damage repair. In this case, p53 acts as a cell survivor. Several p53 target genes, $p 21, G A D D 45$ and 14-3-3 $\sigma$, are involved in p53-dependent cell cycle arrest (el-Deiry 1998).

p21, a cyclin-dependent kinase inhibitor, functions as a regulator for both $\mathrm{G} 1 / \mathrm{S}$ and $\mathrm{G} 2 / \mathrm{M}$ transition. Embryonic fibroblasts derived from p21 knockout mice have defects in their ability to arrest in G1 in response to DNA damage (Brugarolas, Chandrasekaran et al. 1995; Deng, Zhang et al. 1995). Moreover, deletion of p21 accelerates tumor onset in mice expressing a mutant form of $\mathrm{p} 53$ (R172P) that is defective in promoting apoptosis but retains partial growth arrest activity (Barboza, Liu et al. 2006). Microarray studies suggest that inhibition of cell-cycle progression genes is a mechanism of p21-induced cell cycle arrest (Chang, Watanabe et al. 2000). p21 inhibits cell cycle progression at G1 primarily through the inhibition of CDK2 activity, which is required not only for the phosphorylation of RB with the consequent release and activation of E2F-dependent gene expression, but also for the firing of replication origins and for the activity of proteins directly involved in DNA synthesis (Abbas and Dutta 2009). In addition, CDK2 can associate with E2F1 and directly repress its transactivation ability (Delavaine and La Thangue 1999). p21 can also induce G2/M arrest, probably through inhibiting the CDK1-cyclin B1 complex.

By inhibiting cell cycle progression, p21 allows cells to undergo DNA repair. Evidences show that p21 might be involved in DNA repair by interacting with proliferating cell nuclear antigen (PCNA), a DNA polymerase accessory factor, which is important for both DNA replication and DNA repair (Prives and Gottifredi 2008).

GADD45 and 14-3-3 $\sigma$ have also been shown to induce G2/M arrest. GADD45 destabilizes the CDK1-cyclin B1 complex (Zhan, Antinore et al. 1999; Jin, Tong et al. 2002), whereas 
14-3-3 $\sigma$ prevent nuclear import of cyclin B1 and CDK1 through sequestration in the cytoplasm (Chan, Hermeking et al. 1999).

\section{Senescence}

Transient cell cycle arrest might be risky if DNA damage cannot be repaired. Thus, p53 triggers such cells with oncogenic potential to undergo irreversible cell cycle arrest (called senescence) or apoptosis. The phenomenon of senescence was first observed in cell culture, where isolated human diploid fibroblasts could not grow indefinitely (Hayflick and Moorhead 1961). Later, it was shown that telomere shortening mainly contributes to this phenomenon (Harley, Futcher et al. 1990). Telomere dysfunction is sensed as DNA damage signals that activated p53, leading to cellular senescence (Deng, Chan et al. 2008). Oncogene activation was also shown to be capable to induce p53-dependent senescence (Serrano, Lin et al. 1997). Two key mediators of p53-induced senescence are p21 and PAI-1 (Brown, Wei et al. 1997; Kortlever, Higgins et al. 2006; Leal, Fominaya et al. 2008). Interestingly, the senescence program can act together with the innate immune system to limit tumor growth (Xue, Zender et al. 2007).

\section{Apoptosis}

One of the most dramatic functions of p53 is to induce apoptosis. p53 has been implicated to be involved in both extrinsic and intrinsic apoptotic pathways. The extrinsic pathway involves engagement of death receptors, such as Fas, DR5 and PERP, which have been shown to be p53 targets. The intrinsic pathway is in response to stress signals, leading to permeabilization of the outer mitochondrial membrane (OMM), and so the release of cytochrome $c$ and subsequent caspase activation. p53 exerts its apoptotic activity in the intrinsic pathway through both transcription-dependent and -independent manners.

p53 is capable of inducing many pro-apoptotic genes, including those encoding Bcl2 family member Bax, BH3 only proteins Puma and Noxa. Further, p53 can repress the expression of anti-apoptotic genes, for example the one encoding the Bcl2 family member Bcl-2. Puma is an essential mediator of p53-induced apoptosis through the mitochondrial pathway ( $\mathrm{Yu}$ and Zhang 2003). Puma can promote translocation and oligomerization of Bax, leading to 
permeabilization of OMM (Yu, Wang et al. 2003).

p53-induced apoptosis, through the mitochondrial pathway, can also be independent of transactivation. In response to DNA damage, not all p53 molecules are translocated into the nucleus. In contrast, a portion of p53 proteins are translocated into mitochondria. There, p53 interacts with the anti-apoptotic proteins $\mathrm{Bcl}-2$ and $\mathrm{Bcl}-\mathrm{XL}$, leading to the release of the pro-apoptotic proteins Bid and Bax. In addition, it can trigger Bak homo-oligomerization through releasing it from the Bcl-2 family member Mcl-1. Therefore, mitochondrial p53 has dual roles of neutralizing anti-apoptotic proteins as well as activating pro-apoptotic proteins (Moll, Wolff et al. 2005).

One study interconnected the transcriptionally dependent and independent apoptotic function of p53 (Chipuk, Bouchier-Hayes et al. 2005). Under genotoxic stress, cytoplasmic p53 is sequestered by Bcl-XL. Nuclear p53 induces Puma, which then dislodges cytoplasmic p53 from Bcl-XL and activates the transcriptionally independent apoptotic function of cytoplasmic p53.

\section{Cell-fate decisions}

p53 target genes are not universally induced upon the activation of p53. The discriminatory regulation of p53 target genes is needed to achieve the desirable outcome. Although the mechanisms are not well understood, it is clear that p53 levels, its modifications, p53 binding proteins and DNA topology of p53 response elements (REs) can all affect target selection (Vousden and Prives 2009).

As mentioned above, many target genes of p53 contain REs, but not all of them strictly follow the consensus sequence of p53 REs. For example, the binding site of p53 to the PIG3 promoter is a pentanucleotide microsatellite sequence $(\mathrm{TGYCC})_{\mathrm{n}}$ where $\mathrm{Y}=\mathrm{C}$ or $\mathrm{T}$ (Contente, Dittmer et al. 2002). This microsatellite was found to be polymorphic with 10-17 tandem repeats. The DNA topology and sequence of p53 REs may affect p53 binding affinities. In addition to p53 REs, regions proximal to p53 REs might also contribute to differential activation of p53 target genes. For example, the regions proximal to p53 REs of the GADD45 and $M d m 2$ genes exhibit constitutively open, non-nucleosome occupied states (Braastad, Han et al. 2003). It was found that high affinity sites tend to associate with cell cycle arrest-related 
genes, whereas low affinity sites are more frequent in pro-apoptotic genes (Inga, Storici et al. 2002). It suggests that the levels of p53 may have profound effects on target selection and cell fate: low levels of p53 protein tend to induce cell cycle arrest, whereas high levels trigger apoptosis (Laptenko and Prives 2006).

There are a number of proteins that affect p53 activity through modulating the selection of p53 targets. They can be either p53-binding proteins or independent transcription factors in cooperation with $\mathrm{p} 53$. The apoptosis stimulating proteins of p53 (ASSPs) contains three members: ASPP1, ASPP2 and inhibitory ASSP (iASPP). ASPP1 and 2 interact with the DNA-binding domain of $\mathrm{p} 53$, facilitating the binding of p53 to the promoters of pro-apoptotic genes, such as Bax, PUMA and PIG3, but not the $p 21$ promoter (Samuels-Lev, O'Connor et al. 2001). In contrast, iASPP interferes with activation of pro-apoptotic genes by countering the effects of ASSP1 and 2 (Bergamaschi, Samuels et al. 2003).

The Sp1 transcription factor contains a zinc finger-type DNA-binding domain that binds GC-rich motifs with high affinity (Briggs, Kadonaga et al. 1986; Kadonaga, Carner et al. 1987). It functions as a dual activator and repressor of transcription via protein-protein interactions or interplay with other transcription factors (Naar, Ryu et al. 1998). It has been shown that $\mathrm{Sp} 1$, bound to $p 21$ promoter together with $\mathrm{p} 53$, leads to activation of $p 21$ gene transcription (Koutsodontis, Tentes et al. 2001).

The zinc-finger protein Hzf is a p53 target (Sugimoto, Gromley et al. 2006), which can in turn modulate p53 transactivation functions (Das, Raj et al. 2007). Hzf binds to p53 DNA-binding domain, inducing preferential expression of cell cycle arresting p53 target genes, such as $p 21$ and 14-3-3 $\sigma$ genes, over its pro-apoptotic genes. Moreover, Hzf can sense the extent of DNA damage and instruct p53 to switch between apoptosis and growth inhibition.

In addition, post-translational modifications of p53 may also affect target gene selection. p53 conformation is flexible, allowing diverse promoter recognition (Kim and Deppert 2003). Some post-translational modifications, for example phosphorylation and acetylation, might stabilize p53 in a particular conformation that is able to recognize particular types of promoters.

Phosphorylation on Ser46 has a clear role in transactivation of pro-apoptotic genes, such as p53-regulated apoptosis-inducing protein 1 (p53AIP1) (Oda, Arakawa et al. 2000), a 
pro-apoptotic factor that promotes the release of mitochondrial cytochrome $\mathrm{c}$ during apoptosis. Several kinases, HIPK2, DYRK2, protein kinase C delta and p38, have been implicated to phosphorylate this site (Shmueli and Oren 2007). HIPK2 is strictly regulated by Mdm2 (Rinaldo, Prodosmo et al. 2007). Only under high DNA damage stress, HIPK2 is released from the regulation of Mdm2 and is able to phosphorylate p53 at Ser46 to induce apoptosis.

In the core DNA-binding domain of p53, lysine 120 (K120) is acetylated by two MYST family histone acetyltransferases, Tip60 and MOF, in response to DNA damage, resulting in induced PUMA expression (Sykes, Mellert et al. 2006; Tang, Luo et al. 2006). In contrast, when p53 is acetylated at lysine $320(\mathrm{~K} 320)$ in the linker region by the transcriptional coactivators p300 and CREB-binding protein (CBP), it tends to activate p21 rather than pro-apoptotic genes (Knights, Catania et al. 2006).

In addition to the role of p53 target gene selection, the post-translational modifications of p53 have more profound effects on p53 stability and activation, which will be discussed in the next chapter.

\subsubsection{Regulation of p53}

p53 is one protein that has diverse functions. There is no surprise that multiple mechanisms of p53 regulation exist to ensure that p53 can sense different stresses and trigger corresponding outcomes. Although several mechanisms have been proposed to be involved in p53 stabilization and activation, such as N-terminal phosphorylation and C-terminal acetylation of p53, the results from both in vitro biochemical and tissue culture studies and in vivo genetic studies are often inconsistent. But all point out that a negative regulator (Mdm2) of p53 plays a central role in $\mathrm{p} 53$ regulation.

\section{Mdm2, a negative regulator of p53}

The murine double minute $(m d m 2)$ gene, which codes for the Mdm2 protein, is a RING finger protein. It consists of an N-terminal p53 interaction domain, a central acidic domain, and a C-terminal RING domain conferring E3-ubiquitin ligase activity. It is implied as an oncogene, and it has been observed to be amplified in a subset of human cancers expressing wild type 
p53 (Oliner, Kinzler et al. 1992). Genetic studies show that Mdm2 knockout mice die early during the embryonic development but lethality can be rescued by deleting p53 (Jones, Roe et al. 1995; Montes de Oca Luna, Wagner et al. 1995). These data imply that Mdm2 is an essential negative regulator of $\mathrm{p} 53$.

Mdm2 regulates p53 stabilization mainly through its E3-ubiquitin ligase activity. Proteins that are targeted for degradation first have to be ubiquitinated at lysine residues. Mdm2 can specifically ubiquitinate p53 at its C-terminal lysines. Whether Mdm2 induces p53 mono-ubiquitination or polyubiquitination is still in debated. A report shows that low levels of Mdm2 mediate p53 mono-ubiquitination, while higher levels induce poly-ubiquitination (Li, Brooks et al. 2003). Mono- and poly-ubiquitinated p53 have different fates. Only poly-ubiquitinated p53 is subject to subsequent degradation by the proteasome, whereas mono-ubiquitination leads to nuclear export of p53 (Li, Brooks et al. 2003). Thus, in unstressed cells with low levels of Mdm2, another ubiquitin ligase is needed to add poly-ubiquitin chains to the lysines that are previously mono-ubiquitinated by Mdm2. p300, a histone acetyl transferase might be the potential candidate (Grossman, Deato et al. 2003). Furthermore, mono-ubiquitination might promote mitochondrial p53 translocation (Marchenko, Wolff et al. 2007), where it induces apoptosis independent of p53 transactivation ability. p53 in both nuclear and cytoplasmic compartments can be poly-ubiquitinated and degraded, regardless of nuclear export of p53 (Stommel and Wahl 2004).

In addition to its E3-ubiquitin ligase activity, Mdm2 can function as inhibitor of p53 transactivation. It binds to the p53 transactivation domain, thereby preventing the basal transcription macherinary from binding (Momand, Zambetti et al. 1992; Oliner, Pietenpol et al. 1993). Mdm2 can also be recruited to the promoters of p53 target genes and represses their transcription (Minsky and Oren 2004).

As a critical regulator of $\mathrm{p} 53, \mathrm{Mdm} 2$ is tightly controlled. The RING finger protein MdmX, a homologue of Mdm2, lacks E3-ubiquitin ligase activity. MdmX is as critical as Mdm2 in regulating p53 in a non-redundant way since deletion of MdmX in mice also leads to early embryonic lethality, which can be rescued by inactivating p53 (Parant, Chavez-Reyes et al. 2001; Migliorini, Lazzerini Denchi et al. 2002). Through their RING domains, Mdm2 and MdmX can form homo- or hetero-dimers. Mdm2 and MdmX collaborate to regulate p53 
stability. As an E3-ubiquitin ligase, Mdm2 can ubiquitinate and degrade MdmX (Kawai, Wiederschain et al. 2003; Pan and Chen 2003). In turn, MdmX can stabilize both Mdm2 and p53 and promote Mdm2 E3-ubiquitin ligase activity (Linares, Hengstermann et al. 2003; Uldrijan, Pannekoek et al. 2007). MdmX is also capable of repressing p53's transactivation function (Marine and Jochemsen 2005).

p14ARF is an alternate reading frame (ARF) product of the CDKN2A locus. p14ARF is induced by oncogenic activation, modulates DNA damage response, and is frequently mutated in cancer cell lines expressing wild type p53. Several mechanisms have been implied for ARF-mediated regulation of p53. p14ARF is a nucleolar protein and can sequester Mdm2 in the nucleolus, leading to the release of nucleoplasmic p53 (Weber, Taylor et al. 1999). p14ARF can also inhibit Mdm2 E3-ubiquitin ligase activity, and interfere with Mdm2 and p53 interaction (Honda and Yasuda 1999). All contributes to p53 activation.

HAUSP, also known as USP7, is a deubiquitinating enzyme that cleaves ubiquitin from its substrates. It was previously shown that HAUSP can deubiquitinate p53, therefore protecting p53 from Mdm2-mediated degradation (Li, Chen et al. 2002). Likewise, HAUSP can also reverse Mdm2 auto-ubiquitination (Cummins, Rago et al. 2004; Li, Brooks et al. 2004). Thus, Mdm2 and HAUSP form a complicated circuit to fine-tune the stabilization and activity of p53.

The activity and stability of Mdm2 can be also affected by its phosphorylation. p53 can be activated by DNA damage such as double-strand breaks (DSBs). The primary activator of such response is the ATM protein kinase. ATM can phosphorylate a range of players in the DSB response, including p53 and its two main inhibitors Mdm2 and MdmX. One mechanism of p53 activation by phosphorylation of $\mathrm{Mdm} 2$ and $\mathrm{MdmX}$ is that it inhibits $\mathrm{Mdm} 2 / \mathrm{MdmX}$ and $\mathrm{p} 53$ interaction. Another possibility is that ATM-mediated phosphoylation of $\mathrm{Mdm} 2 / \mathrm{MdmX}$ accelerate their degradation, which is dependent on the functional Mdm2 RING domain (Stommel and Wahl 2004). As mentioned above, HAUSP as deubiquitinase can stabilize Mdm2/MdmX. DNA damage-induced phosphorylation of Mdm2/MdmX leads to dissociation of Mdm2/MdmX from HAUSP, resulting in their destabilization (Meulmeester, Maurice et al. 2005). In addition, Mdm2 could be unstable due to enhanced ubiquitination and degradation of MdmX following ATM-mediated phosphorylation of MdmX (Pereg, Shkedy et al. 2005). 


\section{p53 activation by post-translational modifications}

The classic model for stress-induced p53 activation consists of a p53 stabilization step followed by DNA binding and transactivation of p53 target genes. Considering the essential role of Mdm2 in p53 regulation as mentioned above, the stress induces p53 stabilization primarily through interfering with the interaction between p53 and Mdm2. Phosphorylation of p53 is one of the major post-translational modifications, which is considered to inhibit p53 interaction with $\mathrm{Mdm} 2$, thereby preventing Mdm2-mediated ubiquitination and subsequent degradation. The phosphorylation sites of p53 are mainly the serine residues within the N-terminal transactivation domain (Figure 1). These phosphorylations are brought by a number of protein kinases that respond to DNA damage and other stresses including ATM, ATR, the checkpoint kinases Chk1 and Chk2, Jun NH2-terminal kinase (JNK), p38 and others (Lavin and Gueven 2006).

Ser15 of p53 can be directly phosphorylated by ATM and ATR in response to DNA damage (Banin, Moyal et al. 1998; Canman, Lim et al. 1998; Khanna, Keating et al. 1998; Lakin, Hann et al. 1999). Ser15 phosphorylation appears to be a priming event for the subsequent series of modifications (Appella and Anderson 2001). Thr18 and Ser20 are phosphorylated by Chk2, which is activated by ATM (Craig and Hupp 2004). Phosphorylation of Thr18 and Ser20 is proposed to inhibit the association of p53 with Mdm2 (Craig, Burch et al. 1999). Together with Ser15, phosphorylation of these sites increase p53 binding with the histone acetylase family members p300/CBP, leading to C-terminal acetylation of several key lysine residues, which are normally targets for ubiquitination (Lavin and Gueven 2006). Acetylation and ubiquitination are mutually exclusive modifications because it is observed that acetylated p53 cannot be ubiquitinated by Mdm2 in vitro (Ito, Lai et al. 2001; Li, Luo et al. 2002). Thus, stress-induced p53 acetylation promotes p53 stabilization and activation. Another mechanism of acetylation-induced p53 stabilization is that acetylation of p53 can inhibit Mdm2-p53 interaction (Tang, Zhao et al. 2008).

As shown above, both phosphorylation and acetylation stabilize p53 mainly via inhibiting Mdm2-p53 interaction. It is possible to achieve p53 stabilization by merely disrupting Mdm2-p53 interaction regardless of post-translational modifications. Indeed, a small molecule 
called Nutlin-3 was identified as Mdm2 antagonist (Vassilev, Vu et al. 2004). This compound is a cis-imidazoline analog, which binds $\mathrm{Mdm} 2$ in the p53-binding pocket, thus preventing Mdm2-p53 interaction.

Although in vitro assays clearly demonstrate the important roles of phosphorylation and acetylation in p53 activation, knockin mice with mutations in p53 N-terminal phosphorylation sites and $\mathrm{C}$-terminal lysine residues are unable to recapitulate these effects. It suggests that there is regulatory redundancy among post-translational modifications and there might be other unidentified residues that can be post-translationally modified.

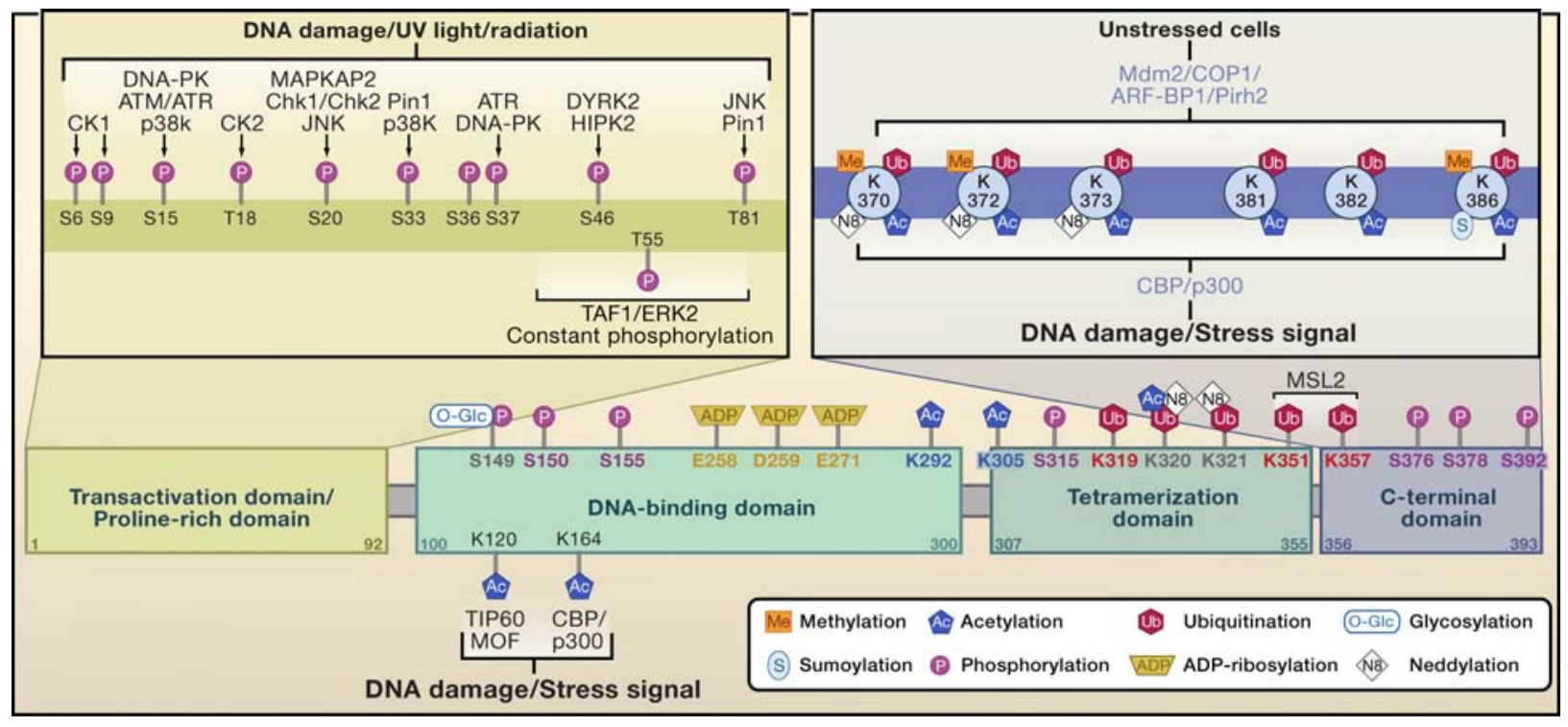

Figure 1. Overview of p53 Posttranslational Modifications

More than 36 amino acids of p53 are reported to be modified. The major sites of p53 phosphorylation (P), ubiquitination ( $\mathrm{Ub})$, and acetylation (Ac) are shown with the corresponding major modifying enzymes and signals. Furthermore, additional phosphorylation and acetylation sites, as well as major sites of methylation (Me), sumoylation (S), neddylation (N8), glycosylation (O-Glc), and ribosylation (ADP), are indicated. Figure and legend from Kruse \& Gu, 2009. 


\section{2 microRNAs, novel gene regulatory molecules}

MicroRNAs (miRNAs) are a class of small ( $\sim 22 \mathrm{nt})$, endogenous non-coding RNAs. They are generated from hairpin-shaped transcripts. The mature microRNAs function as mediators in gene regulation at the post-transcriptional level by base pairing with the target mRNAs, usually in the 3' untranslated region (UTR). More than 30\% protein-coding genes are predicted to be targeted by miRNAs (Lewis, Burge et al. 2005). Binding of a miRNA to the target mRNA usually leads to translational repression and/or mRNA degradation. Other types of regulation by miRNAs, such as gene expression induction (Place, Li et al. 2008) and heterochromatin formation (Kim, Saetrom et al. 2008), have also been reported.

Through interfering with target gene expressions, miRNAs play important roles in almost every important cellular process and aberrant expression of miRNAs is observed in human pathologies, including cancer (Esquela-Kerscher and Slack 2006; Kloosterman and Plasterk 2006).

In this chapter, I will introduce recent findings on miRNA biogenesis and mechanisms of gene regulation by miRNAs. Furthermore, miRNA functions in tumorigenesis, especially in the context of tumor suppressor p53, will be discussed.

\subsection{1 microRNA biogenesis}

The canonical miRNA biogenesis pathway is shown in Figure 2 and will be reviewed in details in the following sections.

\section{miRNA genes and their transcription}

Early annotation of the genomic position of miRNAs indicated that most miRNA genes are located in the intergenic regions ( $>1 \mathrm{~kb}$ away from annotated/predicted genes) (Kim 2005). It implies that most miRNAs are transcribed from their own transcription units. It was also found that approximately $50 \%$ of mammalian miRNA loci are in close proximity to other miRNAs (Kim 2005), and these clustered miRNAs might be transcribed from a single polycistronic transcription unit. However, there are also some exceptional cases. For example, miR-433 and miR-127 are in the miR-433-127 locus, but they arise from independent overlapping primary transcripts (Song and Wang 2008). 


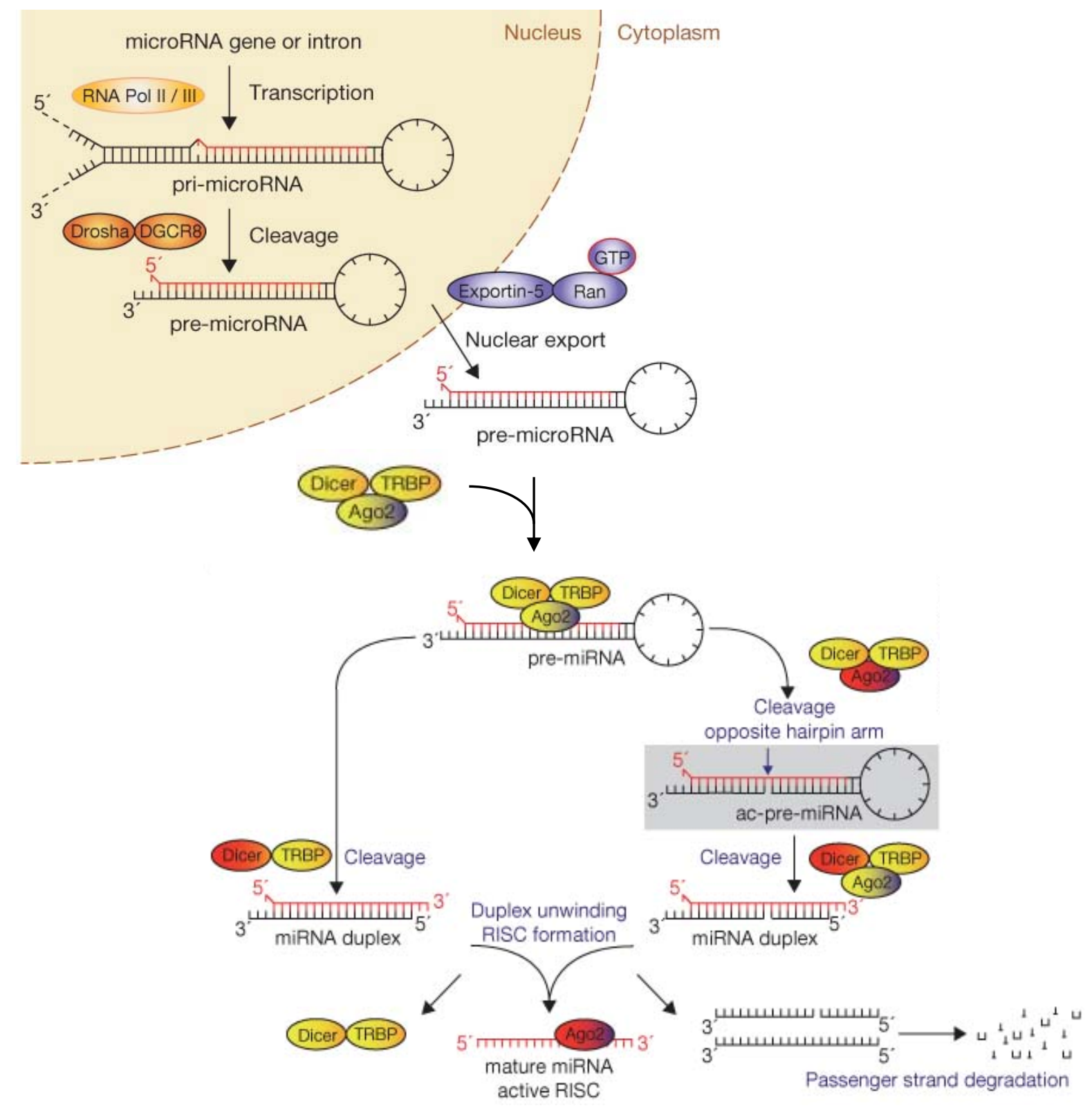

Figure 2. The canonical miRNA biogenesis pathway. The primary miRNA transcript (pri-miRNA) is transcribed by RNA polymerase II or III. It is first cleaved by the microprocessor complex Drosha-DGCR8 in the nucleus to release the hairpin intermediate (pre-miRNA). The resulting pre-miRNA is then exported into the cytoplasm with the aid of Exportin-5. In the cytoplasm, Dicer associates with dsRNA-binding protein TRBP and Ago2 to cleave the pre-miRNA to its mature length. For some miRNAs, Ago2 cleaves the prospective passenger strand preceding Dicer cleavage to generate a nicked hairpin called ac-pre-miRNA. The guide strand of mature miRNA in complex with Agonaute (Ago2) proteins is loaded into RNA-induced silencing complex (RISC), where it guides RISC to target mRNA. Adapted from Winter et al., 2009.

Another miRNA gene location analysis revealed that $\sim 70 \%$ of mammalian miRNA genes are located in defined transcription units (Rodriguez, Griffiths-Jones et al. 2004). These miRNAs can be either in the intronic or exonic region of non-coding transcription units, or 
intronic region of protein-coding transcription units.

Several evidences pointed out that miRNA are transcribed by RNA polymerase II (pol II) (Cai, Hagedorn et al. 2004; Lee, Kim et al. 2004). First, many primary miRNA transcripts (pri-miRNA) are capped and polyadenylated. Second, their transcription is sensitive to the treatment of RNA pol II inhibitor $\alpha$-amanitin. Last, chromatin immunoprecipitation analysis showed direct binding of pol II to the promoter of miR-23a/miR-27a/miR-24-2 cluster. In contrast, miRNAs in the human chromosome 19 miRNA cluster (C19MC) are transcribed by polymerase III(Borchert, Lanier et al. 2006). Pol II-dependent transcription often associates with some transcription factors, for example c-Myc or p53, which allows a subset of miRNA genes to be regulated in a specific way (O'Donnell, Wentzel et al. 2005; He, He et al. 2007).

\section{pri-miRNA cleavage by the Drosha-DGCR8 microprocessor complex}

The pri-miRNA processing in the canonical model is a post-transcriptional event. But recent studies show that pri-miRNA processing is a co-transcriptional process and precedes the splicing of a host intron (Kim and Kim 2007). pri-miRNA cleavage requires nuclear RNase III Drosha and its co-factor DiGeorge syndrome critical region gene 8 (DGCR8) in humans (Pasha in D.melanogaster and C. elegans) (Lee, Ahn et al. 2003; Denli, Tops et al. 2004; Gregory, Yan et al. 2004; Han, Lee et al. 2004). DGCR8 contains two double-stranded RNA binding domains. Together with DGCR8, Drosha forms a large complex known as microprocessor complex, which is $\sim 500 \mathrm{kDa}$ in D.melanogaster (Denli, Tops et al. 2004) and $\sim 650 \mathrm{kDa}$ in humans (Gregory, Yan et al. 2004; Han, Lee et al. 2004). Drosha and DGCR8 have distinct roles in pri-miRNA processing. A typical pri-miRNA consists of a stem of $\sim 33$ base pairs, a terminal loop and flanking single-stranded regions. The flanking regions and the stem can be recognized by DGCR8, which directs Drosha to cleave the substrate $\sim 11$ bp away from the ssRNA-dsRNA junction (Zeng, Cai et al. 2005; Han, Lee et al. 2006). A small short hairpin termed pre-miRNA is then released for further processing.

Recently, it was also shown that Drosha can directly cleave hairpin structures in miRNA (Han, Pedersen et al. 2009). For example, Drosha can negatively regulate its co-factor DGCR8 by cleaving two hairpin structures in the 5'untranslated region and the coding sequence of the Dgcr8 mRNA (Han, Pedersen et al. 2009). 
Drosha-mediated cleavage can be regulated in many ways to facilitate miRNA-specific regulation. In addition to the core proteins of microprocessor complex Drosha and DGCR8, several other proteins can be part of the complex. For example, the RNA helicases p72 and p68 are part of the complex and might act as specificity factors for the processing of a subset of pri-miRNAs (Gregory, Yan et al. 2004). miR-21 is induced in response to bone morphogenetic protein (BMP)/transforming growth factor- $\beta$ (TGF $\beta$ ) signaling (Davis, Hilyard et al. 2008). It was proposed that SMAD proteins activated by BMP/TGF $\beta$ bind to the pri-miR-21 in complex with p68 and stimulate pri-miR-21 processing. The heterogeneous nuclear ribonucleoprotein A1 (hnRNP A1) binds specifically to pri-miRNA-18a and facilitates its processing, without affecting other miRNAs that are located in the same miR-17 genomic cluster (Guil and Caceres 2007). The primary transcript of let-7 is expressed in both undifferented and differentiated ES cells, whereas mature let-7 is detectable only in differentiated cells (Thomson, Newman et al. 2006; Wulczyn, Smirnova et al. 2007). One mechanism for let-7 post-translational regulation is that an RNA-binding protein, Lin-28, can block Drosha processing (Newman, Thomson et al. 2008).

There are some exceptional cases where Drosha is not required for pri-miRNA processing (Okamura, Hagen et al. 2007; Ruby, Jan et al. 2007). Such miRNAs (called mirtrons) are derived from small introns. After splicing, the branched mirtrons are resolved and form hairpin structures that resemble premiRNAs.

\section{Nuclear export by Exportin 5}

After nuclear processing by Drosha, pre-miRNAs are exported to the cytoplasm by the aid of Exportin 5 in complex with GTP-bound form of the co-factor Ran (Yi, Qin et al. 2003). Exportin 5 is a member of the nuclear transport receptor family, and it was originally known to be a minor export factor for tRNAs (Bohnsack, Regener et al. 2002; Calado, Treichel et al. 2002). Considering much higher affinity of Exportin 5 to pre-miRNAs, the main cargo of Exportin 5 might be pre-miRNAs. The length of the double-stranded stem and 3'overhangs can be recognized by Exportin 5 for successful binding (Lund, Guttinger et al. 2004; Zeng and Cullen 2004). Knockdown of Exportin 5 decreases the abundance of mature miRNAs, but does not accumulate pre-miRNAs, which implies that association of the pre-miRNA to Exportin 5 
can stabilize itself (Yi, Qin et al. 2003; Lund, Guttinger et al. 2004).

\section{Cytoplasmic processing by Dicer}

After exporting to the cytoplasm, the pre-miRNA is further processed into $22 \mathrm{nt}$ miRNA duplex by another RNase III enzyme, called Dicer (Bernstein, Caudy et al. 2001; Grishok, Pasquinelli et al. 2001; Hutvagner, McLachlan et al. 2001; Ketting, Fischer et al. 2001). Dicer was originally known to initiate the RNA interference (RNAi) pathway (Bernstein, Caudy et al. 2001). Dicer is a highly conserved protein found in almost all eukaryotic organisms (Bernstein, Caudy et al. 2001). The human Dicer interacts with two closely related proteins, TRBP (TAR RNA-binding protein) (Chendrimada, Gregory et al. 2005) and PACT, and the core component Argonaute-2 (Ago2) (Lee, Hur et al. 2006). This complex is called RISC (RNA-induced silencing complex) loading complex (RLC), which mediates pre-miRNA processing and RISC assembly. The main effector in RLC is Dicer, and TRBP and PACT only facilitate it (Chendrimada, Gregory et al. 2005; Lee, Hur et al. 2006). For some miRNAs, another endonucleolytic cleavage step precedes Dicer-mediated cleavage, where Ago2 cleaves the prospective passenger strand to generate a nicked hairpin called ac-pre-miRNA (Diederichs and Haber 2007). The Ago2-mediated cleavage might facilitate the subsequent strand dissociation and RISC activation.

Considering the important role of Dicer in miRNA biogenesis, it could be a critical step for the regulation of miRNA expression. For example, Dicer mRNA can be targeted be its product let-7, providing a feedback loop (Forman, Legesse-Miller et al. 2008). In addition, beyond its effect on nuclear microprocessor activity, Lin-28 was shown to inhibit Dicer cleavage in vitro to prevent pre-let-7 maturation (Rybak, Fuchs et al. 2008). Furthermore, the pre-let-7 is polyuridylated by a terminal uridylyl transferase in the presence of Lin-28 (Heo, Joo et al. 2008). The uridylated pre-let-7 bypasses Dicer processing and is degraded by nucleases.

\section{Guide strand selection and Argonaute loading}

The resulting miRNA duplex after Dicer cleavage needs to be separated into the functional guide strand, which is loaded into RISC and guides the complex to target mRNAs, and the passenger strand, which is degraded (Schwarz, Hutvagner et al. 2003). A universal helicase 
responsible for miRNA duplex unwinding has not been identified yet, and it may require specific helicase to regulate miRNAs specifically (Winter, Jung et al. 2009). As discussed above, Ago2-mediated cleavage of pre-miRNAs facilitates strand dissociation, which implies that helicases might not be indispensable (Diederichs and Haber 2007). The relative thermodynamic stability of the base pairs at the two ends of the duplex determines which strand is to be selected (Khvorova, Reynolds et al. 2003; Schwarz, Hutvagner et al. 2003). The strand with relative less stable base pair at its 5'end is loaded into RISC (Khvorova, Reynolds et al. 2003; Schwarz, Hutvagner et al. 2003).

In D. melanogaster, the structure of miRNA duplex determines small RNA sorting into RISC with different Ago proteins (Forstemann, Horwich et al. 2007; Tomari, Du et al. 2007). miRNA duplexes with central mismatches are preferentially sorted into Ago1, whereas fully complementary siRNA duplexes are sorted into Ago2 (Forstemann, Horwich et al. 2007; Tomari, Du et al. 2007). In human, Agol to 4 are biologically and biochemically distinct, but the patterns of associated miRNAs with different Ago proteins are almost identical (Liu, Carmell et al. 2004; Meister, Landthaler et al. 2004; Azuma-Mukai, Oguri et al. 2008).

\subsubsection{Mechanisms of miRNA-directed gene regulation}

As novel regulators of gene expression, miRNAs mainly mediate post-transcriptional gene silencing in the cytoplasm. Together with Argonaute family proteins, miRNAs assemble into RISC and guide Argonaute proteins to target mRNAs, which are either translationally repressed or degraded. Exceptional cases were also observed that miRNAs can switch from repression to activation of target mRNA translation under certain conditions. Furthermore, accumulating evidences imply that miRNAs can be imported into the nucleus and regulate gene expression at the transcriptional level.

\section{Post-transcriptional regulation by miRNAs}

The mechanisms of post-transcriptional gene silencing by miRNAs are still under debate. Until now, no unique and common silencing mechanism has been proposed. In contrast, multiple regulation modes (Figure 3), some of which are contradictory, are raised from different 


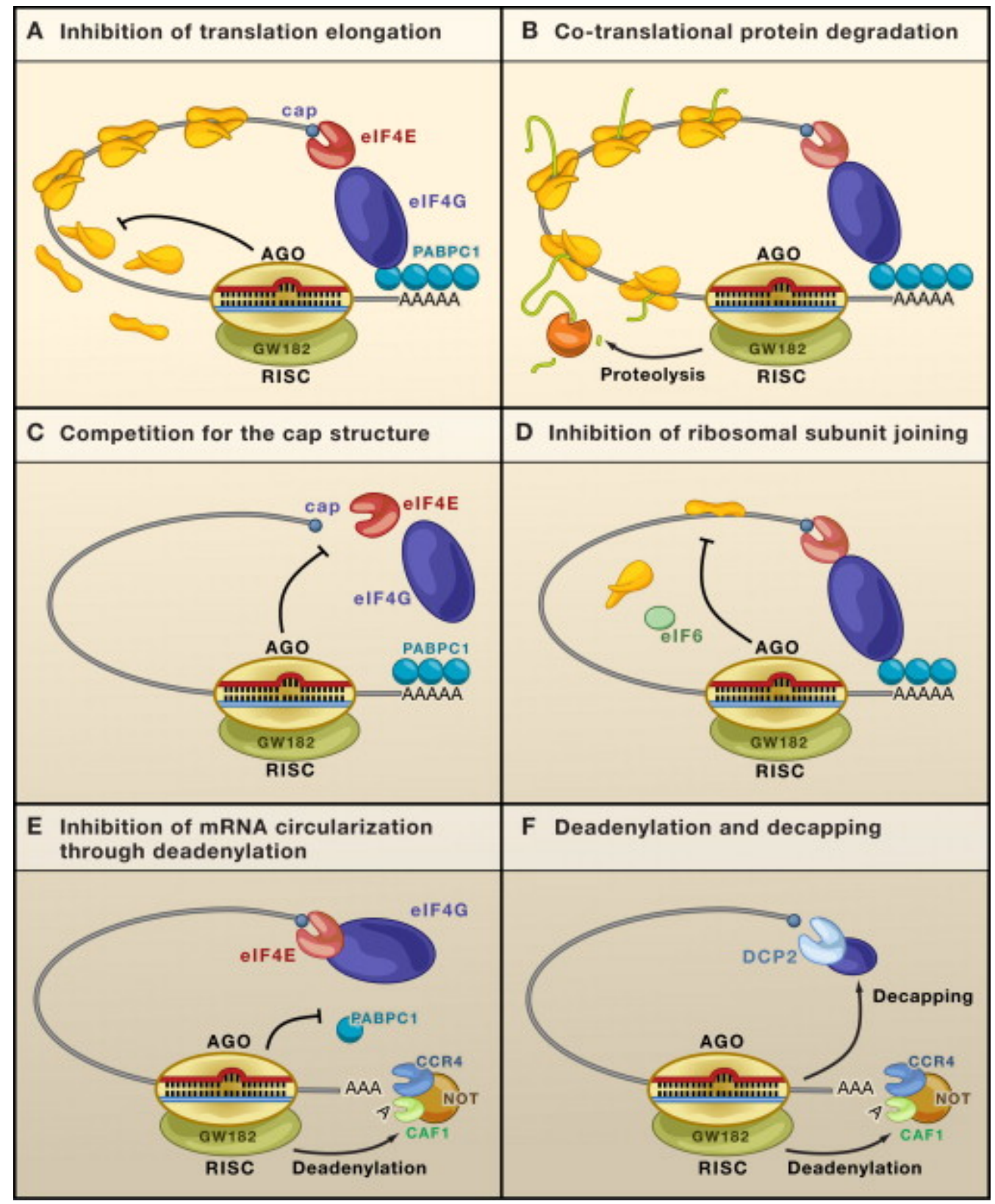

Figure 3. Mechanisms of post-translational silencing by miRNAs (Eulalio, Huntzinger et al. 2008).

(A) Post-initiation repression. miRNAs silence target mRNAs by blocking translation elongation or promoting premature ribosome dissociation.

(B) Co-translational protein degradation. Targets mRNAs are actively transcribed, but the nascent polypeptides are degraded co-translationally.

(C-E) Initiation repression. (C) Ago proteins compete with eIF4G for cap binding. (D) Ago-associated protein GW182 recruit eIF6, preventing 60S subunit from joining the $40 \mathrm{~S}$ subunit. (E) Through deadenylation, Ago proteins prevent circularization of target mRNAs, which in turn decreases the affinity of eIF4E for cap binding.

(F) miRNA-induced mRNA decay. miRNAs promote deadenylation and subsequent decapping of target mRNAs. 
experimental approaches. mRNA translation consists of three steps: initiation, elongation and termination. miRNAs can apply the repression effects on all these steps. In addition, miRNAs can induce target mRNA degradation despite imperfect miRNA-mRNA base pairing. miRNAs might also silence their targets by sequesting mRNAs in discrete cytoplasmic foci known as mRNA processing bodies or P bodies, which exclude the translation machinery (Eulalio, Huntzinger et al. 2008).

\section{Repression at the initiation step}

Polysome gradient analysis revealed that miRNAs and their targets are not associated with the polysomal fraction in sucrose gradients, indicating that the repression occurs at the initiation step (Pillai, Bhattacharyya et al. 2005). Reporter mRNA analysis further supports the idea of a repression effect on the initiation step: the translation of $\mathrm{m}^{7} \mathrm{G}$-capped mRNAs, but not of mRNAs containing an internal ribosome entry site (IRES) or a non-functional ApppN cap, is repressed by miRNAs (Humphreys, Westman et al. 2005; Pillai, Bhattacharyya et al. 2005). One study implied that the central domain of Argonaute proteins share sequence homology to the cap-binding protein eIF4E (eukaryotic translation initiation factor 4E) (Kiriakidou, Tan et al. 2007), which is important for the cap-dependent translation initiation. Another study showed that adding purified eIF4E to the ascites extract rescued the miRNA-induced inhibition (Mathonnet, Fabian et al. 2007). These results support the idea that miRNA-directed Ago proteins can compete with eIF4E for $\mathrm{m}^{7} \mathrm{G}$ cap binding and thus prevent translation of capped mRNAs.

Co-immunoprecipitation analysis indicated that eIF6 and 60S ribosomal subunit proteins associate with the Ago2-Dicer-TRBP complex (Chendrimada, Finn et al. 2007). eIF6 is involved in $60 \mathrm{~S}$ subunit biogenesis in the nucleolus, and accompanies the 60S subunit to the cytoplasm (Sanvito, Piatti et al. 1999; Si and Maitra 1999; Basu, Si et al. 2001). Knockdown of eIF6 in either human cells or C. elegans rescues mRNA targets repressed by miRNA, suggesting Argonaute proteins repress translation by recruiting eIF6, which prevents the $60 \mathrm{~S}$ subunit from joining to the 40S subunit (Chendrimada, Finn et al. 2007).

$\underline{\text { Repression at the post-initiation steps }}$ 
In contrast, the polysome gradient analysis in human cells and C. elegans from other studies demonstrated that repressed mRNAs are associated with actively translating polysomes (Seggerson, Tang et al. 2002; Maroney, Yu et al. 2006; Nottrott, Simard et al. 2006; Petersen, Bordeleau et al. 2006), supporting the idea that miRNAs inhibit mRNA translation at steps after initiation. Additional evidence shows that reporter mRNAs containing IRES are also repressed by miRNAs (Petersen, Bordeleau et al. 2006).

Based on this mode, the target mRNAs should be actively transcribed. However, the nascent polypeptides are not detected (Nottrott, Simard et al. 2006; Petersen, Bordeleau et al. 2006), which might be due to cotranslational degradation. In order to confirm this, the putative proteases should be identified. Proteasome inhibitors had no effects on miRNA-mediated repression (Nottrott, Simard et al. 2006).

\section{miRNA-induced mRNA decay}

mRNA decay is another inhibitory mechanism for miRNAs, instead of translational repression. Based on this observation, microarrays have been widely used to identify target genes of specific miRNAs, although it is not applicable to translationally repressed genes. In plants, most miRNA-mRNA duplexes have nearly perfect base pair complementarity, which induces mRNA degradation through the RNAi-like mechanism. In contrast, metazoan miRNA-mRNA duplexes contain central bulges and mismatches, precluding the Argonaute-mediated endonucleolytic cleavage of mRNAs. Studies in zebrafish embryos, C. elegans, D. melanogaster, and human cells showed that miRNAs accelerate deadenylation and decapping of target mRNAs (Wu and Belasco 2005; Behm-Ansmant, Rehwinkel et al. 2006; Giraldez, Mishima et al. 2006; Eulalio, Rehwinkel et al. 2007), suggesting that miRNAs induce mRNA decay through the general mRNA degradation machinery.

In eukaryotes, mRNA degradation is initiated by a gradual shortening of the mRNA poly (A) tail, a process called deadenylation. The mRNA is then degraded by progressive 3 ' $\rightarrow 5^{\prime}$ ' decay, which is catalysed by the exosome, or by the removal of the cap followed by 5 ' $\rightarrow 3^{\prime}$ degradation, which is catalysed by the exonuclease XRN1 (Filipowicz, Bhattacharyya et al. 2008). A key factor, the GW182 protein, was identified for promoting mRNA deadenylation (Behm-Ansmant, Rehwinkel et al. 2006). GW182 was shown to interact with Ago1, and 
depletion of GW182 or Ago1 leads to the upregulation of a common set of target mRNAs (Behm-Ansmant, Rehwinkel et al. 2006). Moreover, depletion of the components of the CCR4-NOT deadenylating complex interferes with GW182-promoted mRNA decay. The decapping enzymes DCP1 and DCP2 are also shown to be involved in miRNA-mediated degradation (Behm-Ansmant, Rehwinkel et al. 2006; Eulalio, Rehwinkel et al. 2007). In summary, the mechanism of miRNA-induced mRNA decay is proposed: miRNA-directed Ago1 recruits GW182, which then recruits the deadenylase complex CCR4-NOT to remove the mRNA poly (A) tail, followed by removal of the $\mathrm{m}^{7} \mathrm{G}$ cap by decapping enzymes DCP1 and DCP2.

\section{$\underline{\text { Translation activation by miRNAs }}$}

As mentioned above, the dominant effects of miRNAs, including translational repression and mRNA decay, are all negative. Few cases have reported the "positive" function of miRNAs. One study showed that miR-122 has stimulatory effects on replication of Hepatitis C Virus (HCV) RNA in liver cells (Jopling, Yi et al. 2005). Another group reported that Ago2 and FXR1, two microRNA ribonucleoproteins (RNP), can bind to the AU-rich elements (ARE) in the 3'UTR of TNF $\alpha$ mRNA, unexpectedly activating its translation in a cell-growth-dependent manner (Vasudevan and Steitz 2007). Further study by this group indicated that miR369-3 directs association of these proteins with the ARE to activate translation (Vasudevan, Tong et al. 2007). Two other well-studied miRNAs, let-7 and the synthetic miRcxcr4 likewise oscillate between repression and activation during the cell cycle (Vasudevan, Tong et al. 2007). However, the mechanisms are not known yet.

\section{Transcriptional gene regulation by miRNAs}

In order to regulate gene expression at the transcriptional level, miRNAs first need to be re-imported into the nucleus. One study on miR-29b revealed that it is predominantly localized to the nucleus, and the hexanucleotide terminal motif of miR-29b acts as a transferable nuclear localization element (Hwang, Wentzel et al. 2007). NRDE-3, a member of the C. elegans Argonaute family, is considered to be the transporter because of its role in siRNA transporting from the cytoplasm to the nucleus (Guang, Bochner et al. 2008). Importin-8, the RAN-GTP 
shuttle protein, affects the nuclear Ago2 pool in human cells (Weinmann, Hock et al. 2009).

Studies showed that miRNAs can modulate gene transcription in both a suppressive and activating manner. miR-320 has a cis-regulatory in transcriptional silencing of the cell cycle gene POLR3D expression (Kim, Saetrom et al. 2008). It recruits Ago1, Polycomb group (PcG) protein EZH2, and tri-methyl histone H3 lysine 27 (H3K27me3) to the POLR3D promoter, which provides an epigenetic mechanism of transcriptional silencing by miRNAs in mammalian cells.

The mechanisms of RNA-induced transcriptional silencing (RITS) have been intensively studied for siRNAs, which might drop some hints on how miRNAs direct transcriptional silencing. RITS is carried out by a protein complex called the RITS complex, consisting of argonaute, a chromodomain protein Chp1, and an argonaute interacting protein Tas 3 in fission yeast (Verdel, Jia et al. 2004). The RITS complex has been shown to be involved in the initiation and spreading of heterochromatin in the mating-type region and in centromere formation in the fission yeast. The maintenance of heterochromatin regions by the RITS complex has been described as a self-reinforcing feedback loop, in which the RITS complex associates with siRNAs complementary to the local genes, stably binds the methylated histones and co-transcriptionally degrades any nascent mRNA transcripts, which are then used as RNA-dependent RNA polymerase substrates to replenish the complement of siRNA molecules (Sugiyama, Cam et al. 2005). Heterochromatin formation, but possibly not maintenance, is Dicer-dependent, because Dicer is required for generating the initial complement of siRNAs that target subsequent transcripts (Noma, Sugiyama et al. 2004).

One group reported that both siRNAs and miRNAs that are complementary to a promoter can increase gene transcription, a phenomenon called RNA activation (RNAa) (Li, Okino et al. 2006; Place, Li et al. 2008). Compared to RNAi that has silencing effects for 5 to 7 days, RNAa activates gene expression for up to 13 days. This process requires the Ago 2 protein and associates with the loss of histone 3 methylation at lysine-9. Since the exact mechanism is unknown at present, many people are suspecting that RNAa is just the downstream effect of RNAi. 


\subsection{3 microRNAs and cancer}

More than $30 \%$ of human genes are predicted to be targeted by miRNAs (Lewis, Burge et al. 2005), and many of these genes are involved in cell differentiation, proliferation and apoptosis, suggesting a role of miRNAs in cancer pathogenesis. miRNA gene location analysis further revealed that half of the known miRNAs are located at fragile sites and genomic regions involved in cancers (Calin, Sevignani et al. 2004). For example, the miR-15a/miR-16-1 cluster is located in the $13 \mathrm{q} 14$ region, which is deleted in $>50 \%$ of human cases with chronic lymphocytic leukemia (CLL) (Dohner, Stilgenbauer et al. 2000; Bullrich, Fujii et al. 2001); the miR-17-92 cluster is located in the 13q31 region, which is commonly amplified in lymphoma (Ota, Tagawa et al. 2004). miRNA expression profiles also demonstrated dysregulation of miRNAs in diverse cancers (Calin, Ferracin et al. 2005; Iorio, Ferracin et al. 2005; Lu, Getz et al. 2005; Volinia, Calin et al. 2006; Yanaihara, Caplen et al. 2006).

The miRNA expression profiles can clearly differentiate human cancers, and only a few miRNAs are needed to accurately predict the origin of tumor tissue ( $\mathrm{Lu}$, Getz et al. 2005). These miRNA expression changes are not simply due to a secondary consequence of malignant transformation. Rather, the loss- or gain-of-function of specific miRNAs plays important roles in tumorigenesis.

\section{miRNAs as oncogenes and tumor suppressors}

Accumulated evidences clearly showed that specific miRNAs have tumor-suppressing or tumor-promoting activity, acting as tumor suppressors or oncogenes. The following identification and verification of cancer-relevant targets demonstrated the mechanisms through which these miRNAs participate in tumorigenesis. Here, I will give two examples in which miRNA let-7 and miR-372/-373 have been well-characterized as tumor suppressor and oncogene individually.

The miRNA let-7 is frequently reduced in lung cancer, and this reduction is associated with decreased survival and poor prognosis (Takamizawa, Konishi et al. 2004; Yanaihara, Caplen et al. 2006). Overexpression of let-7 in A549 cells, a lung adenocarcinoma cell line, was observed to inhibit cell proliferation (Takamizawa, Konishi et al. 2004). Two oncogenes RAS 
(KRAS and NRAS) and MYC were confirmed to be the direct targets of let-7 (Johnson, Grosshans et al. 2005; Sampson, Rong et al. 2007). The lung tumor tissues display significantly reduced levels of let-7 and significantly enhanced levels of RAS compared to normal lung tissue, which further suggests that let-7 controls lung cancer development through negative regulation of RAS (Johnson, Grosshans et al. 2005).

In contrast to let-7, miR-372/-373 was found to function as oncogenes in human testicular germ cell tumors (Voorhoeve, le Sage et al. 2006). In response to oncogenic activation of RAS, p53 is activated and induces cellular senescence. By using a miRNA expression library, Agami and co-workers performed a cell-based screen and identified two miRNAs (miR-372/-373) that could neutralize p53 function and allow continued proliferation in the presence of activated RAS. These two miRNAs are frequently overexpressed in testicular germ cell tumors. Through repressing Large Tumor Suppressor homolog 2 (LATS2), miR-372/-373 can release p53-mediated CDK2 inhibition, resulting in continued cell proliferation.

\section{miRNAs and the $\mathbf{p} 53$ pathway}

p53-responsive miRNAs

As discussed in the p53 section, p53 acts as a transcription factor, which can activate or repress many downstream target genes. Most miRNAs are transcribed by RNA polymerase II, which always associates with transcription factors, for example c-Myc or $\mathrm{p} 53$, to regulate specific gene expression. Thus, p53 was proposed to regulate not only protein-coding genes but also miRNA-coding genes. Indeed, reports from several groups claimed that the members of the miR-34 family are direct p53 targets, which induce apoptosis, cell cycle arrest and senescence (Bommer, Gerin et al. 2007; Chang, Wentzel et al. 2007; Corney, Flesken-Nikitin et al. 2007; He, He et al. 2007; Raver-Shapira, Marciano et al. 2007; Tarasov, Jung et al. 2007; Tazawa, Tsuchiya et al. 2007).

The miR-34 family contains three miRNAs (miR-34a, miR-34b and miR-34c) encoded from two different gene loci: miR-34a located in chromosome 1 has its own transcript, whereas miR-34b and miR-34c located in chromosome 11 are produced from a common primary transcript (Raver-Shapira and Oren 2007). The chromosome region 1p36 encoding miR-34a is subject to hemizygous deletions in neuroblastoma, and reduced miR-34a expression is often 
observed in tumors with such deletion (Welch, Chen et al. 2007). Likewise, the region encoding miR-34b and miR-34c is often deleted in cancer (Calin, Sevignani et al. 2004).

Both miR-34a and miR-34b/c loci contain consensus p53-binding sites (Bommer, Gerin et al. 2007). Chromatin immunoprecipitation and promoter luciferase assay further confirmed p53 binding to the promoters of both genes (Bommer, Gerin et al. 2007; Chang, Wentzel et al. 2007; He, He et al. 2007; Raver-Shapira, Marciano et al. 2007). All these miRNAs can be induced by DNA damage and oncogenic stress in a p53-dependent manner (Bommer, Gerin et al. 2007; He, He et al. 2007; Raver-Shapira, Marciano et al. 2007). The outcomes of such activation include apoptosis, cell cycle arrest and senescence, which largely depend on the cell type examined. Thus, the miR-34 family acts as p53 downstream effector. Furthermore, miR-34a was shown to have a positive feedback on $\mathrm{p} 53$, which might be due to down-regulation of the E2F pathway (Tazawa, Tsuchiya et al. 2007).

In order to define the mechanism by that miR-34 family exerts pro-apoptotic and antiproliferative activity, the targets of the miR-34 family should be identified. miR-34a and miR-34c have identical seed sequences and in principle target the same mRNAs, whereas the seed sequence of miR-34b is similar but not identical (Raver-Shapira and Oren 2007). One gene expression microarray analysis revealed that induction of miR-34a leads to widespread alterations in gene expression (Chang, Wentzel et al. 2007). Genes classified as "cell cycle", "DNA repair", "mitotic checkpoint" and "DNA integrity checkpoint" were highly enriched among the up-regulated transcripts, while genes classified as "cell proliferation" and "angiogenesis" were significantly enriched among the down-regulated genes. Interestingly, another microarray analysis implied that more than half of the down-regulated genes were also among the repressed gene list of adriamycin (a DNA damaging agent) treated cells (He, He et al. 2007). Thus, the repression of many genes by DNA damage might be due to miR-34a induction by p53. Several genes, including CDK4, Cyclin E2, MET and Bcl-2, have been validated to be miR-34a targets (Bommer, Gerin et al. 2007; He, He et al. 2007; Tazawa, Tsuchiya et al. 2007). Knockdown of CDK4, Cyclin E2, MET can phenocopy miR-34a induced G1 arrest (He, He et al. 2007), and Bcl-2 is a well-documented anti-apoptotic gene.

In summary, miR-34 family members are the first identified p53-responsive miRNAs. miR-34 family members are not only downstream effectors of p53 by regulating anti-apoptotic 
and pro-apoptotic genes, but also upstream regulators of p53 probably by down-regulating the E2F pathway.

miRNA regulators of the $\mathrm{p} 53$ pathway

p53 is a master regulator in response to multiple cellular stresses. To control and fine-tune the responses, p53 is tightly regulated at the transcriptional level and especially at the post-translational level. miRNAs provide a novel layer of gene regulation, and might be involved in the regulation of the tumor suppressor $\mathrm{p} 53$.

Using computational prediction, one group discovered that miR-125b targets both human and zebrafish p53 3'UTR (Le, Teh et al. 2009). miR-125b has been shown to promote neuronal differentiation in human cells (Le, Xie et al. 2009). Overexpression of miR-125b represses endogenous p53 and suppresses apoptosis in human neuroblastoma cells and human lung fibroblasts. Knockdown of miR-125b in turn enhances p53 protein levels and induces apoptosis in human lung fibroblasts. The biological functions of miR-125b were also checked in zebrafish. Interestingly, there is a reverse correlation between miR-125b and p53 expression during zebrafish embryogenesis. Knockdown of miR-125b leads to severe defects in the zebrafish embryo, for example the accumulation of dead cells in the brain. In line with this, knockdown of miR-125b in zebrafish embryo leads to increased p53 and p53-induced apoptosis. Furthermore, overexpression of miR-125b represses p53 induction and stress-induced apoptosis when zebrafish embryos are treated with $\gamma$-irridiation or camptothecin. Together, these data suggest that miR-125b is a negative regulator of $\mathrm{p} 53$.

In contrast, another group reported that miR-29 family members (miR-29a, miR-29b and miR-29c) activate p53 and elevate p53-induced apoptosis by targeting p85a (the regulatory subunit of PI3 kinase) and CDC42 (a Rho family GTPase) (Park, Lee et al. 2009).

In addition to affecting p53, other components of the p53 pathway might be targets of miRNAs. Indeed, the miR-106b family members were shown to trigger G1/S transition, partly by negatively regulating the cyclin-dependent kinase inhibitor p21/CDK1A, a well known p53 target (Ivanovska, Ball et al. 2008). 


\subsection{Aims of the study}

Accumulating evidences implicated important roles of miRNAs in tumorigenesis. Specific miRNAs are known to regulate cell cycle progression and apoptosis, which are overlapped effects exerted by the tumor suppressor p53. We proposed that miRNAs might constitute part of the p53 tumor suppressor network and contribute to its biological activities. Therefore, the main goal of this study was to determine how miRNAs function in the p53 tumor suppressor network.

Specifically, we aimed to address two issues:

1. Identification and characterization of miRNAs that are regulated by the transcription factor p53. Most miRNA genes are transcribed by RNA Pol II, which is always associated with specific transcription factors, such as c-Myc, E2F or p53, to regulate specific gene expression. Thus, p53 might also regulate protein non-coding miRNA genes. Indeed, miR-34 family members have been shown to be the downstream targets of p53 (see last section). In addition to miR-34a, we also found that the two clusters encoding miR-192, miR-194, and miR-215 were p53 responsive. The biological functions of miR-192/215 in the context of p53 were further characterized in this study.

2. Identification and characterization of miRNAs that are upstream regulators of p53. p53 stability and activity are tightly regulated at its post-transcriptional levels. miRNAs provide a novel layer of gene regulation in a manner of post-translational repression, and they might affect p53 directly or indirectly. In a cell-based screening approach, we isolated two miRNAs (miR-22 and miR-101) that could regulate p53. miR-22 reduced the levels and activities of $\mathrm{p} 53$. We aimed to define the mechanisms and consequences of $\mathrm{p} 53$ attenuation by miR-22. miR-101 expression accumulated modified p53, probably mono-ubiquitinated p53. We aimed to define what post-transcriptional modification on p53 is induced by miR-22 and to explain how this modification is induced.

Taken together, this study was to identify novel miRNAs, which function as effectors and/or regulators of $\mathrm{p} 53$. 


\section{Materials}

\subsection{Reagents}

Acrylamide

Roth

Agar

Roth

Agarose (electrophoresis grade)

GibcoBRL

Ammoniumpersulfat (APS)

Roth

Ampicillin

Sigma

Blasticidin $\mathrm{S} \mathrm{HCl}$

Invitrogen

Bovine growth serum

HyClone

Bovine serum albumin (BSA)

Roth

Bromphenol blue

Sigma

Calcium chloride $(\mathrm{CaCl} 2)$

Roth

Camptothecin

Sigma

Chloroform

Roth

Ciprobay

Bayer

dNTPs, $25 \mu \mathrm{mol}$ each

Promega

Dimethylsulphoxide (DMSO)

Applichem

Dithiothreitol (DTT)

Roth

Doxorubicin

Sigma

EDTA

Roth

Ethanol

Roth

Ethidiumbromid

Roth

Formaldehyde

Sigma

GeneRuler DNALadder Mix

Fermentas

Glycerin

Roth

Glycin

Roth

HiPerfect transfection Reagent

Qiagen

Hoechst 33342

Invitrogen 
Isopropanol

Laminin

Lipofectamine 2000

Magnesium chloride $(\mathrm{MgCl} 2)$

Methanol

Milk powder

Nocodazole

Nonidet P40 substitute

Nuclease-free water

PageRuler Prest. Protein Ladder

Protease inhibitor cocktail

(Aprotinin/Leupeptin/Pefabloc)

Restriction enzymes

RNase A

Roti®-Phenol

Sepharose CL-4B

Sodium Chloride

Sodium deoxycholate

Sodium dodecylsulfate (SDS)

TEMED

Tetracyclin

Tris

Triton X-100

Tween 20

T4 DNA ligase

Urea

5-Fluorouracil

2-Mercaptoethanol
Roth

Sigma

Invitrogen

Roth

Roth

Roth

Sigma

Amersham

Ambion

Fermentas

Roth

New England Biolabs

Fermentas

Qiagen

Roth

Sigma

Roth

AppliChem

Roth

Roth

Sigma

Roth

AppliChem

AppliChem

NEB

Roth

Sigma

Roth 


\subsection{Media}

\subsubsection{Media for bacterial culture:}

$\underline{\text { LB (Luria-Bertani) medium : }}$

- 10 g peptone

$-5 \mathrm{~g}$ yeast extract

$-10 \mathrm{~g} \mathrm{NaCl}$

- adjust the $\mathrm{pH}$ to 7 with $\mathrm{NaOH}$ and add $\mathrm{ddH}_{2} \mathrm{O}$ to $1 \mathrm{~L}$

- sterilize at $121^{\circ} \mathrm{C}$ for $15 \mathrm{~min}$

2xYT:

- 16 g peptone

- 10 g yeast extract

$-5 \mathrm{~g} \mathrm{NaCl}$

- adjust the $\mathrm{pH}$ to 7 with $\mathrm{NaOH}$ and add $\mathrm{ddH}_{2} \mathrm{O}$ to $1 \mathrm{~L}$

- sterilize at $121^{\circ} \mathrm{C}$ for $15 \mathrm{~min}$

For plates, $1.5 \%$ agar was added. Antibiotics ampicillin $(200 \mu \mathrm{g} / \mathrm{ml})$ or tetracyclin $(25 \mu \mathrm{g} / \mathrm{ml})$ were used.

\subsubsection{Media for cell culture:}

Dulbecco's Modified Eagle's Medium (DMEM):

- 10 g DMEM powder, GIBCO

$-3.7 \mathrm{~g} \mathrm{NaHCO}_{3}$

- 5.96 g HEPES

- add $\mathrm{ddH}_{2} \mathrm{O}$ to $1 \mathrm{~L}$

- filtered, autoclaved and stored at $4{ }^{\circ} \mathrm{C}$

RPMI 1640:

- RPMI 1640 (1x) liquid, GIBCO 


\section{McCoy's 5A:}

- McCoy's 5A (1x) liquid, GIBCO

Supplements for DMEM full, RPMI full and McCoy's 5A full:

- 10\% fetal calf serum (FCS)

- 2 mM L-glutamin

- 50 units/ml penicillin

- $50 \mu \mathrm{g} / \mathrm{ml}$ streptomycin

$-2 \mu \mathrm{g} / \mathrm{ml}$ tetracyclin

- $10 \mu \mathrm{g} / \mathrm{ml}$ ciprobay

\subsection{Buffers}

Crystal violet solution (500ml):

- $125 \mathrm{ml}$ methanol

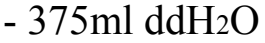

- $0.5 \mathrm{~g}$ crystal violet

DNA staining buffer for FACS:

- $0.5 \mathrm{mg} / \mathrm{ml}$ RNase A, $30 \mu \mathrm{g} / \mathrm{ml}$ propidium iodide (PI) in PBS ++

\section{EB buffer:}

- $10 \mathrm{mM}$ Tris $(\mathrm{pH} 8.5)$

Immunoprecipitation (IP) buffer:

- $50 \mathrm{mM}$ Tris $\mathrm{HCl} \mathrm{pH} 7.5$

$-300 \mathrm{mM} \mathrm{NaCl}$

- 1\% Nonidet P40 (v/v)

- $0.5 \%$ Sodium Deoxycholate 
IP lysis buffer:

- Aprotinin/Leupeptin (1:1000), Pepstatin (1:1000), Pefa Bloc (1:100) in IP buffer

Laemmli buffer 6x:

- 0.35 mM Tris-HCl pH 6.8

- 30\% Glycerol (v/v)

$-10 \% \operatorname{SDS}(\mathrm{w} / \mathrm{v})$

$-9.3 \%$ DTT $(w / v)$

- $0.02 \%$ Bromphenol blue (w/v)

Lysis buffer for SDS-PAGE:

- 2M Urea in RIPA buffer

- Protease inhibitor cocktail (Aprotinin/Leupeptin 1:1000, Pepstatin 1:1000, Pefa Bloc 1:100)

$\underline{\text { PBS }++ \text { (Phosphate Buffered Saline) }}$ :

- $236.9 \mathrm{mM} \mathrm{NaCl}$

$-2.7 \mathrm{mM} \mathrm{KCl}$

- $8.1 \mathrm{mM} \mathrm{Na} 2 \mathrm{HPO}_{4}$

- $1.5 \mathrm{mM} \mathrm{KH}_{2} \mathrm{PO}_{4}$

$-1.1 \mathrm{mM} \mathrm{MgCl} 2$

$-1.2 \mathrm{mM} \mathrm{CaCl} 2$

- adjust to $\mathrm{pH} 7.4$

$\underline{\mathrm{PBS}^{\mathrm{def}}}:$

- Composition and preparation as for $\mathrm{PBS}++$ but without $\mathrm{MgCl}_{2}$ and $\mathrm{CaCl}_{2}$

PBS-T:

$-0.1 \%$ Tween $20(\mathrm{v} / \mathrm{v})$ in PBS

Ponceau S-solution: 
- add $0.5 \mathrm{~g}$ Ponceau $\mathrm{S}$ to $1 \mathrm{ml}$ acetic acid, mix

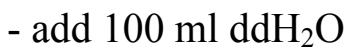

RIPA buffer:

- 20 mM Tris-HCl pH 7.5

- $10 \mathrm{mM}$ EDTA

- $150 \mathrm{mM} \mathrm{NaCl}$

- $1 \%$ Sodium deoxycholate $(\mathrm{w} / \mathrm{v})$

$-0.1 \% \operatorname{SDS}(\mathrm{w} / \mathrm{v})$

- 1\% Triton-X $100(\mathrm{v} / \mathrm{v})$

- 10 mM Iodacetamide

- $5 \%$ Trasylol (v/v) (equals 100,000 KIE)

\section{SDS-PAGE running buffer:}

$-25 \mathrm{mM}$ Tris

- 192 mM Glycine

$-0.1 \% \operatorname{SDS}(\mathrm{w} / \mathrm{v})$

Stripping buffer:

- $50 \mathrm{ml}$ Tris $1 \mathrm{M}, \mathrm{pH} 6.8$

- $400 \mathrm{ml} \mathrm{10 \%} \mathrm{SDS}$

- $10 \mathrm{ml} \beta$-Mercaptoethanol

- $540 \mathrm{ml} \mathrm{ddH} \mathrm{H}_{2} \mathrm{O}$

TAE (Tris-Acetate-EDTA)- running buffer for agarose-gel electrophoresis, 50x:

- 242 g Tris base

- $57.1 \mathrm{ml}$ acetic acid

- $100 \mathrm{ml}$ 0.5M EDTA pH8.0

- add $\mathrm{ddH}_{2} \mathrm{O}$ to $1 \mathrm{~L}$ 
TBS (Tris Buffered Saline, 10x):

$-90 \mathrm{~g} \mathrm{NaCl}$

- $61 \mathrm{~g}$ Tris

- add $\mathrm{ddH}_{2} \mathrm{O}$ to $1 \mathrm{~L}$

- adjust to $\mathrm{pH} 7.4$

TBS-T:

$-0.1 \%$ Tween20 $(\mathrm{v} / \mathrm{v})$ in TBS

Transfer buffer for Western blot:

- 100 ml 10x Western salts

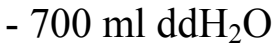

- $200 \mathrm{ml}$ methanol

Western salts(10x):

$-250 \mathrm{mM}$ Tris

- 1.92 M Glycine

- adjust to $\mathrm{pH} 8.3$

\subsection{Antibodies}

Primary antibodies:

$\begin{array}{lllll}\text { Name } & \text { Source } & \text { Producer } & \text { Application } & \text { Dilution } \\ \text { Anti- } \beta \text {-actin } & \text { Rb pAb } & \text { Abcam } & \text { WB } & 1: 5000 \\ \text { Anti- } \beta \text {-gal } & \text { Ms mAb } & \text { Promega } & \text { IP } & 1 \mu \text { ger reaction } \\ \text { Anti-cleaved caspase 3 } & \text { Rb mAb } & \text { Cell signaling } & \text { WB } & 1: 1000 \\ \text { Anti-Cyclin B1 } & \text { Ms mAb } & \text { Abcam } & \text { WB } & 1: 1000 \\ \text { Anti-Chk2 } & \text { Ms mAb } & \text { Santa Cruz } & \text { WB } & 1: 1000 \\ \text { Anti-EZH2 } & \text { Ms mAb } & \text { Cell signaling } & \text { WB } & 1: 1000 \\ & & & \text { IP } & 1: 50\end{array}$




\begin{tabular}{|c|c|c|c|c|}
\hline Anti-Mdm2 2A9 & Ms mAb & Hybridoma & WB & $1: 500$ \\
\hline & & Supernatant & & \\
\hline \multirow[t]{2}{*}{ Anti-Mdm2 2A10 } & Ms mAb & Hybridoma & WB & $1: 500$ \\
\hline & & Supernatant & & \\
\hline Anti-Noxa & Ms mAb & Oncogene & WB & $1: 1000$ \\
\hline \multirow[t]{2}{*}{ Anti-p21 } & Ms mAb & Calbiochem & WB & $1: 1000$ \\
\hline & & & IF & $1: 200$ \\
\hline \multirow[t]{3}{*}{ Anti-p53 (DO-1) } & Ms mAb & Santa Cruz & WB & $1: 1000$ \\
\hline & & & IP & $1 \mu \mathrm{g}$ per reaction \\
\hline & & & IF & $1: 200$ \\
\hline Anti-p53 (DO-1) HRP & Ms mAb & Santa Cruz & WB & $1: 1000$ \\
\hline Anti-acetyl-p53 (Lys382) & $\mathrm{Rb} \mathrm{pAb}$ & Cell signaling & WB & $1: 1000$ \\
\hline Anti-phospho-p53 (Ser15) & Ms mAb & Cell signaling & WB & $1: 1000$ \\
\hline Anti-phospho-p53 (Ser20) & Ms mAb & Cell signaling & WB & $1: 1000$ \\
\hline Anti-PARP-1 & Ms mAb & Calbiochem & WB & $1: 1000$ \\
\hline Name & Source & Producer & Application & Dilution \\
\hline Anti-phospho-Chk2 (T68) & $\mathrm{Rb} \mathrm{pAb}$ & Cell Signaling & WB & $1: 1000$ \\
\hline Anti-phospho-Histone & Ms mAb & Millipore & WB & $1: 4000$ \\
\hline \multicolumn{5}{|l|}{$\mathrm{H} 2 \mathrm{AX}$} \\
\hline Anti-phospho-Histone H3 & Ms mAb & Cell Signaling & WB & $1: 1000$ \\
\hline Anti-Puma/bbc3, & $\mathrm{Rb} \mathrm{pAb}$ & Sigma-Aldrich & WB & $1: 1000$ \\
\hline \multicolumn{5}{|l|}{ C-terminal } \\
\hline Anti-Sp1 & $\mathrm{Rb} \mathrm{pAb}$ & Millipore & WB & $1: 500$ \\
\hline
\end{tabular}

Secondary antibodies:

Peroxidase-conjugated AffiniPure goat anti-mouse IgG $(\mathrm{H}+\mathrm{L}) \quad$ Jackson Immunoresearch Peroxidase-conjugated AffiniPure donkey anti-rabbit Jackson Immunoresearch Alexa Fluor® 546 goat anti-mouse IgG $(\mathrm{H}+\mathrm{L})$ Molecular Probes 


\subsection{Kits}

Kit

BCA Protein Assay Kit

DNA-free ${ }^{\mathrm{TM}} \mathrm{Kit}$

DNeasy Blood \& Tissue Kit

Dual-Luciferase ${ }^{\circledR}$ Reporter Assay System

Immobilion ${ }^{\circledR}$ Western Chemiluminescent HRP Substrates

Invisorb $^{\circledR}$ Spin Plasmid Mini Two

$\mathrm{iQ}^{\mathrm{TM}} \mathrm{SYBR}^{\circledR}$ Green Supermix

iScript $^{\mathrm{TM}}$ cDNA Synthesis Kit

$\operatorname{mirV} a^{\mathrm{TM}}$ miRNA Isolation Kit

PureYield ${ }^{\text {TM }}$ Plasmid Midiprep System

QIAamp $^{\circledR}$ DNA mini kit

QIAquick ${ }^{\circledR}$ gel extraction kit

QIAquick $^{\circledR}$ PCR purification kit

SuperSignal West Dura Extended Duration Substrate

SuperSignal West Femto Maximum Sensitivity Substrate

TaqMan ${ }^{\circledR}$ MicroRNA Reverse Transcription Kit
Producer

Pierce

Ambion

Qiagen

Promega

Millipore

Invitek

Bio-Rad

Bio-Rad

Ambion

Promega

Qiagen

Qiagen

Qiagen

Pierce

Pierce

Applied Biosystems

\subsection{Bacteria}

Escherichia coli DH10B

Invitrogen

\subsection{Eukaryotic cell lines}

A549, human non-small cell lung adenocarcinoma cells, p53 wild type

DLD-1, human colon carcinoma cells, p53 mutant (S241F)

H1299, human non-small cell lung adenocarcinoma cells, p53 null

HCC2998, human colon carcinoma cells, p53 nonsense mutant (R213*)

HCT116, human colon carcinoma cells, p53 wild type and their counterparts p53-/- and p21-/- 
HT-29, human colon adenocarcinoma grade II cells, p53 mutant (R273H)

LoVo, human colon carcinoma cells, p53 wild type

MCF-7, human breast adenocarcinoma cells, p53 wild type

SJSA, human osteosarcoma cells, p53 wild type

U2OS, human osteosarcoma cells, p53 wild type

\subsection{Plasmids}

miR-Vec (generous gift of R. Agami, see Voorhoeve et al., 2006) is a vector-Based miRNA Expression System.

pSuper-p53 (Brummelkamp et al., 2002) is a system for stable expression of short interfering RNA against $\mathrm{p} 53$.

pMIR-REPORT ${ }^{\mathrm{TM}}$ Luciferase miRNA Expression Reporter Vector (Ambion) is designed for cloning and testing of putative miRNA binding sites. To verify whether miRNAs target directly p53 3'UTR, p53 3'UTR sequence was first amplified by PCR, using primers 5'-AATGCACTAGTTCAAGACAGAAGGGCCTGAC and 5'-GCATTAAGCTTTTCACCC CTCAGACACACAG. Then the PCR fragment was cloned into pMIR-REPORT ${ }^{\mathrm{TM}}$ Luciferase miRNA Expression Reporter Vector using SpeI and HindIII.

SwitchGear UTR Reporter Vector (SwitchGear Genomics) is also used for testing of putative miRNA binding sites. CDKN1A 3'UTR vector, which contains p21 3'UTR sequence, was purchased from SwitchGear Genomics. 


\section{Methods}

\subsection{Cell Biology}

\subsubsection{Cell Culture}

\section{Maintenance of cell culture}

H1299, HT-29, MCF-7, SJSA and U2OS cells were maintained in Dulbecco's Modified Eagle medium (DMEM), DLD-1, HCC2998 and LoVo cells in RPMI1640 medium, and HCT116 cells in McCoy's 5A medium. Media were supplemented with 10\% FCS. All cell lines were cultivated in cell culture flasks or dishes at $37^{\circ} \mathrm{C}$ and $5 \% \mathrm{CO}_{2}$.

To passage the cells, the medium was removed, and cells were washed once with PBS ${ }^{\text {def }}$. Then cells were incubated with $0.05 \%$ Trypsin-EDTA to induce the detachment from the culture flasks or dishes. Cells were carefully resuspended in fresh medium and distributed into new flasks or dishes. For experiments, suspended cells were counted using a counting chamber (Neubauer), and the required number of cells was seeded into corresponding well plates.

\section{Freezing and thawing of cells}

For freezing, a $175-\mathrm{cm}^{2}$ flask with confluent cells was used to obtain 3 aliquots.

Cells were washed with PBS $^{\text {def }}$ and trypsinized. Suspended cells were spinned down for 5 min at $800 \mathrm{rpm}$. Cell pellet was then resuspended in a cold solution of $10 \%$ DMSO in FCS and aliquoted into pre-cooled cryovials. Aliquots were stored at $-80^{\circ} \mathrm{C}$ for 24 to 48 hours and then transferred to liquid nitrogen.

For thawing cells, an aliquot was thawed by hand and was transferred immediately into the flak or dish with fresh warm medium. After one day of incubation at $37^{\circ} \mathrm{C}$, the culture medium was changed.

\subsubsection{Transient transfection of Eucaryotic cells}

For each cell line, the choice of the transfection methods was determined by the transfection efficiency, the toxicity of transfection reagents and the molecules to be delivered. 
For plasmid transfection, Lipofectamine 2000 was normally used. Cells were seeded in a 6-well or 12-well plate one day before the transfection, and incubated at $37^{\circ} \mathrm{C}$. For an example of 6-well plate, $3.2 \mu \mathrm{g}$ DNA (if necessary, the empty vector pcDNA3 was added to achieve ideal amount of DNA) was mixed in $250 \mu \mathrm{l}$ medium. In the meantime, $10 \mu 1$ of Lipofectamine 2000 were mixed in $250 \mu \mathrm{l}$ medium. Both were incubated for $5 \mathrm{~min}$ at RT. Then the lipofectamine mixture was added to the DNA and incubated for another $20 \mathrm{~min}$ at RT. Afterwards, the transfection mixture was transferred to each well. After transfection, cells were incubated at $37^{\circ} \mathrm{C} .4$ to 6 hours post-transfection, medium was changed. Cells were harvested or fixed 24 to 72 hours later, depending on the purpose of the experiments.

For siRNA or synthetic miRNA transfection, reverse transfection was used. The preparation for the transfection mixture was the same as mentioned above. The amount of siRNA or synthetic miRNA depends on each experiment. And only $5 \mu \mathrm{l}$ of Lipofectamine 2000 was needed for each transfection in a 6-well plate. Transfection mixture was first added into the empty well. Then counted cells were added on top of the transfection mixture. 24 hours post-transfection, medium was changed.

\subsubsection{Stable transfection of Eucaryotic cells}

The transfection procedure is described as above. Cells were maintained in non-selective medium for one day post-transfection. Cells were then cultured in selective medium containing the drug for $10-14$ days. Every three or four days medium was changed. In the case of my study, the miR-vector encodes blasticidine resistant gene. Thus, $5 \mu \mathrm{g} / \mathrm{ml}$ blasticidine was used for selection.

\subsubsection{Crystal Violet Staining}

Cell-seeded plates were placed on ice, and washed twice with ice-cold PBS ${ }^{\text {def }}$. Cells were fixed with ice-cold methanol for $10 \mathrm{~min}$. Then, methanol was removed, and crystal violet solution was added and incubated for $10 \mathrm{~min}$ at RT. Finally, plates were carefully rinsed with $\mathrm{ddH}_{2} \mathrm{O}$ and dried at RT. 


\subsubsection{Fluorescence-activated cell sorting analysis (FACS)}

Attached cells, combined with the floating cells, were harvested from 6-well plates. Cell pellet was resuspended in $500 \mu \mathrm{l}$ ice-cold PBS++. $500 \mu \mathrm{l}$ of $100 \%$ ethanol was added in drops to cell suspension three times. Cells were stored overnight at $4{ }^{\circ} \mathrm{C}$. Afterwards, cells were spinned down and washed with $1 \mathrm{ml}$ PBS++. Cells were resuspended in $500 \mu \mathrm{PBS}++$ containing 0.5 $\mathrm{mg} / \mathrm{ml} \mathrm{RNase} \mathrm{A}$ and $30 \mu \mathrm{g} / \mathrm{ml}$ propidium iodide and incubated for $30 \mathrm{~min}$ at $37{ }^{\circ} \mathrm{C}$. Samples were then analyzed by Guava EasyCyte Plus System. Cell cycle analysis was carried out by the ModFit software.

\subsection{Molecular Biology}

\subsubsection{Transformation of E.coli}

There are two methods to transform E. coli with plasmid DNA: Chemical transformation and electroporation. Compared to Chemical transformation, electroporation is more efficient. $0.3 \mu 1$ of plasmid DNA was gently mixed with $7 \mu$ l electrocompetent E.coli (DH10B, Invitrogen). The mixture was then transferred to a pre-chilled $0.1 \mathrm{~cm}$ Gene-Pulser-cuvette (BioRad). The electroporation was done on the Gene-Pulser-Controller, at $1.7 \mathrm{kV}, 200 \Omega$ and $25 \mu \mathrm{F}$. Then, $200 \mu \mathrm{LB}$ or $2 \mathrm{xYT}$ medium was added to the cuvette to suspend bacteria. For selection with Kanamycin, the bacteria suspension was first incubated for 1 hour at $37{ }^{\circ} \mathrm{C}$. Bacteria suspension was plated on an agar dish (with ampicillin or kanamycin) and incubated overnight at $37^{\circ} \mathrm{C}$.

\subsubsection{DNA isolation}

\section{Plasmid preparation from bacteria}

Plasmid preparation from bacteria is based on the alkaline lysis method. According to the size of bacterial culture and plasmid yield, different kits were used.

For plasmid preparation from $2 \mathrm{ml}$ bacteria culture, QIAamp ${ }^{\circledR}$ DNA mini kit (Qiagen) or Invisorb ${ }^{\circledR}$ Spin Plasmid Mini Two (Invitek) was used. 
For plasmid preparation from $15-25 \mathrm{ml}$ bacteria culture, PureYield ${ }^{\mathrm{TM}}$ Plasmid Midiprep System kit (Promega) was used.

All the kits were used according to the manual's instructions.

Isolation of genomic DNA

To purify genomic DNA from human cells, DNeasy Blood \& Tissue Kit (Qiagen) was used.

Cell pellet (maximum $5 \times 10^{6}$ ) was suspended in $200 \mu 1 \mathrm{PBS}^{\mathrm{def}}$, and lysed with $20 \mu 1$ proteinase $\mathrm{K}$ for $10 \mathrm{~min}$ at $56^{\circ} \mathrm{C}$. Genomic DNA was bound to the DNeasy membrane, washed and eluted in Buffer AE (10 mM Tris-HCL; 0.5 mM EDTA pH 9.0).

\subsubsection{Measurement of DNA / RNA concentration}

The concentration and quality of DNA or RNA samples were checked by NanoDrop 1000 Spectrophotometer (Peqlab). $1.5-2 \mu$ samples were pipetted onto the pedestal of the Spectrophotometer. The concentration of DNA or RNA was measured according to the absorbance at $260 \mathrm{~nm}$. The sample purity was checked by $260 / 280$ and 260/230 ratios.

\subsubsection{Polymerase chain reaction (PCR)}

PCR is a technique to amplify target DNA sequence. The method relies on thermal cycling, consisting of repeated heating and cooling of the reaction for DNA melting and enzymatic replication of the DNA.

Example of a PCR reaction:

DNA template

Forward primer $(10 \mu \mathrm{M})$

Reverse primer $(10 \mu \mathrm{M})$

dNTPs (20 mM)

$10 \times$ PCR buffer

Taq polymerase

$\mathrm{H}_{2} \mathrm{O}$
$1 \mu 1$

$5 \mu 1$

$5 \mu 1$

$0.5 \mu 1$

$5 \mu 1$

$0.5 \mu 1$

add to $50 \mu \mathrm{l}$ 
PCR cycles:

\begin{tabular}{|l|l|l|l|l|l|l|}
\hline \multirow{2}{*}{ Step } & \multirow{2}{*}{ Hold } & \multicolumn{2}{|l|}{ Cycle $(30$ cycles $)$} & Hold & \multirow{2}{*}{ Hold } \\
\cline { 3 - 5 } & & Denature & Anneal & Extend & & \\
\hline Time & 3 min & 1 min & 90 sec & 2 min & 5 min & $\infty$ \\
\hline Temp & $94^{\circ} \mathrm{C}$ & $94^{\circ} \mathrm{C}$ & $55^{\circ} \mathrm{C}$ & $72^{\circ} \mathrm{C}$ & $72^{\circ} \mathrm{C}$ & $4^{\circ} \mathrm{C}$ \\
\hline
\end{tabular}

The PCR products were analyzed by agarose gel electrophoresis. If only the desired band was amplified, the PCR product was purified directly by using QIAquick ${ }^{\circledR}$ PCR purification kit (Qiagen). If unspecific bands were also present, the desired band was cut from the gel and purified by using QIAquick® gel extraction kit (Qiagen).

\subsubsection{DNA restriction digestion}

This procedure is used to prepare DNA for cloning or to search for correct clones. The DNA restriction enzymes are isolated primarily from bacteria, and they are able to cleave DNA at specific sites. The digestion time varies from 2 hours to overnight. If the restriction site is at the termini of DNA fragments, incubation of the reaction overnight is preferred. For double digestion, digestion buffer should be carefully chosen to be compatible for both enzymes.

Example of a restriction digestion:

$\begin{array}{ll}10 \times \text { buffer } & 2 \mu \mathrm{l} \\ 10 \times \text { BSA (if necessary) } & 2 \mu \mathrm{l} \\ \text { PCR product/insert/vector } & 5 \mu \mathrm{l} \\ \text { Restriction enzyme } & 2.5 \mu \mathrm{l} \\ \mathrm{H}_{2} \mathrm{O} & \text { add to } 20 \mu \mathrm{l}\end{array}$

Incubate for 2 hours at $37^{\circ} \mathrm{C}$.

\subsubsection{Dephosphorylation of DNA}

To avoid self-ligation of vectors during cloning, the digested vectors were treated with alkaline phosphatase to remove the phosphate groups at the 5 ' end. Simply, $3 \mu 1$ calf intestine phosphatase $(1 \mathrm{u} / \mu \mathrm{l})$ was added to the restriction reaction and incubated for 1 hour at $37^{\circ} \mathrm{C}$. 


\subsubsection{Phenol extraction of DNA}

During cloning, this step is commonly used to purify DNA from enzymes and buffers. Briefly, one volume of phenol was added to the DNA solution. Mixture was vigorously mixed and centrifuged (1 min at full speed). The upper phase, containing the DNA, was transferred to a new tube and mixed with one volume of CIA (chloroform-isoamyl-alcohol 24:1). Mixture was spinned shortly, and the upper phage containing the DNA was transferred into a new tube.

\subsubsection{DNA ethanol precipitation}

DNA solution was mixed with $1 \mu$ l Glycogen, 1/10 volume of $3 \mathrm{M} \mathrm{NaAc}$ and 2.5 volume of EtOH. Mixture was incubated at $-80{ }^{\circ} \mathrm{C}$ for at least 2 hours, followed by centrifugation for 15 min at full speed at $4{ }^{\circ} \mathrm{C}$. DNA pellet was washed with $200 \mu 170 \% \mathrm{EtOH}$, dried and dissolved in $20 \mu \mathrm{l} \mathrm{EB}$ buffer.

\subsubsection{DNA ligation}

T4 DNA ligase was used to form two covalent phosphodiester bonds between 3' hydroxyl end and 5 ' phosphate end of digested insert and vector. For efficient ligation, the amount of insert should be much higher than that of vector.

Example of a DNA ligation reaction:

$\begin{array}{ll}\text { Insert } & 5 \mu 1 \\ \text { Vector } & 1 \mu 1 \\ 10 \text { x buffer } & 1 \mu 1 \\ \text { T4 ligase }(1 \text { weiss/ } \mu 1) & 3 \mu 1 \\ \text { cubate overnight at RT. } & \end{array}$

\subsubsection{DNA sequencing}

The principle of DNA sequencing we used here is based on the chain-terminator method. The polymerization reaction requires a DNA template, a DNA primer, a DNA polymerase, and a mixture of dNTPs and ddNTPs (each of the four ddNTPs labelled with different fluorescent 
dyes). Since ddNTPs do not have the 3'-OH group, its corporation into the newly synthesized strand will terminate the elongation, resulting in DNA fragments of varying length. After capillary electrophoresis to separate the fragments, the use of a laser and the analysis of the fluorescence of the fragments allowed us to read the DNA sequence.

Polymerization reaction mixture:

300 ng template

8 pmol 5' primer or 3' primer

$1.5 \mu 1$ Seqmix

$1.5 \mu 1$ Seqbuffer

Add $\mathrm{ddH}_{2} \mathrm{O}$ to $10 \mu \mathrm{l}$

$\underline{\text { Sequencing program: }}$

\begin{tabular}{|l|l|l|l|l|l|}
\hline Step & \multicolumn{4}{|l|}{ Cycle $(25$ cycles $)$} & \\
\hline Temperature & $96{ }^{\circ} \mathrm{C}$ & $96{ }^{\circ} \mathrm{C}$ & $55^{\circ} \mathrm{C}$ & $60{ }^{\circ} \mathrm{C}$ & $12{ }^{\circ} \mathrm{C}$ \\
\hline Time & $2 \mathrm{~min}$ & $10 \mathrm{sec}$ & $15 \mathrm{sec}$ & $4 \mathrm{~min}$ & pause \\
\hline
\end{tabular}

After polymerization reaction, DNA products were purified by follows: first, the DNA products were transferred to a $1.5 \mathrm{ml}$ tube. Second, $1 \mu 1125 \mathrm{mM}$ EDTA, $1 \mu 13 \mathrm{M} \mathrm{NaAc}$ and $50 \mu 1100 \%$ ethanol were added and mixed gently. Mixture was then incubated for 5 min and centrifuged at $14.000 \mathrm{rpm}$ for $15 \mathrm{~min}$. DNA pellet was washed once with $70 \mu 170 \%$ ethanol, and air-dried for at least 2 min. Finally, DNA products were resuspended in $15 \mu 1$ HiDye. The purified DNA product was ready for sequencing by using 3130x/Genetic Analyzer (Applied Biosystems).

\subsubsection{RNA isolation}

Total RNA, including microRNA was isolated by using the mirVana RNA Isolation kit (Ambion). The kit combines the advantage of organic extraction and solid-phage extraction. Briefly, cells were lysed in $300-600 \mu 1$ Lysis/Binding Solution. 1/10 volume of miRNA Homogenate Additive was added to the cell lysate and incubated for $10 \mathrm{~min}$ at $4{ }^{\circ} \mathrm{C}$. The lysate 
is then extracted once with one volume of Acid-Phenol: Chloroform. The semi-pure RNA sample was mixed with 1.25 volume of $100 \%$ ethanol and applied to a silica membrane. The membrane was washed once with $700 \mu \mathrm{l}$ Wash Solution 1 and twice with $500 \mu 1$ Wash Solution $2 / 3$. RNA was eluted in $95{ }^{\circ} \mathrm{C}$ Nuclease-free water.

In order to avoid genomic DNA contaminant, RNA sample was digested with rDNase I by using DNA-free ${ }^{\mathrm{TM}}$ Kit (Ambion). Briefly, RNA sample was mixed with 0.1 volume $10 \mathrm{x}$ rDNase I Buffer and $1 \mu 1 \mathrm{rDNase} I$ in a $50 \mu 1$ reaction, and incubated for $20-30 \mathrm{~min}$ at $37{ }^{\circ} \mathrm{C}$. Then resuspended DNase Inactivation Reagent was added, and incubated for 2 min at RT. After centrifugation, supernatant which contains the RNA was transferred to a new tube.

\subsubsection{Quantitative RT-PCR}

RT-PCR is a highly sensitive technique enabling amplification of a specific nucleic acid sequence and quantification of the PCR product in real time. In our study, this technique was used for gene expression analysis.

RT-PCR was done in a two-step reaction. First, the RNA was reverse transcribed into cDNA using oligo-dT, random oligomers, or gene-specific primers. An aliquot of the cDNA product was then subsequently added to the real-time PCR.

\section{$\underline{\text { Reverse transcription }}$}

Reverse transcription was performed, using either iScript ${ }^{\mathrm{TM}}$ cDNA Synthesis Kit (Bio-Rad) or home-made Do-It-Yourself Reverse Transcription protocol.

1) Reverse transcription using iScript ${ }^{\mathrm{TM}} \mathrm{cDNA}$ Synthesis Kit

iScript is a modified MMLV-derived reverse transcriptase, pre-blended with RNAse inhibitor. The primers used in the kit are a blend of oligo-dT and random hexamer.

Reaction Set Up 


\section{1 reaction}

$5 *$ iScript Reaction Mix $\quad 4 \mu 1$

iScript RT $\quad 1 \mu 1$

RNA template $\quad 100$ fg to $1 \mu \mathrm{g}$ total RNA

Nuclease-free water $\quad$ up to $20 \mu 1$

The reaction mix was incubated at $25^{\circ} \mathrm{C}$ for $5 \mathrm{~min}, 42{ }^{\circ} \mathrm{C}$ for $30 \mathrm{~min}, 85^{\circ} \mathrm{C}$ for $5 \mathrm{~min}$ and then held at $4{ }^{\circ} \mathrm{C}$. Synthesized cDNA was stored at $-20{ }^{\circ} \mathrm{C}$.

2) Do-It-Yourself Reverse Transcription protocol:

The following components were mixed (per one reaction):

$-1 \mu \mathrm{g}$ of total RNA

- $2 \mu \mathrm{l}$ of combined primer stock ( $15 \mu \mathrm{M}$ nonamers and $50 \mu \mathrm{M}$ oligo $\left.\mathrm{dT}_{23} \mathrm{VN}\right)$

- $4 \mu \mathrm{l}$ of dNTP mix (2.5 mM of each dCTP, dATP, dTTP, dGTP)

- DEPC water to $16 \mu \mathrm{l}$

The mix was heated at $70{ }^{\circ} \mathrm{C}$ for $5 \mathrm{~min}$, spinned down briefly, and put on ice.

Then, the other components were mixed (per one reaction):

- $2 \mu 110$ x Reaction buffer

- $0.25 \mu \mathrm{l}$ of RNase Inhibitor (10 U)

- $0.125 \mu \mathrm{l}$ of M-MuLV Reverse Transcriptase (25 U)

$-1.625 \mu l$ of DEPC water

Reaction was incubated at $42{ }^{\circ} \mathrm{C}$ for $1 \mathrm{~h}$. Reverse transcriptase was inactivated at $95{ }^{\circ} \mathrm{C}$ for 5 min. cDNA was diluted by adding $30^{\circ} \mathrm{C}$ water and stored at $-20^{\circ} \mathrm{C}$.

\section{$\underline{\text { Real-time PCR }}$}

Real-time PCR was performed, using either $\mathrm{iQ}^{\mathrm{TM}} \mathrm{SYBR}^{\circledR}$ Green Supermix (Bio-Rad) or home-made PCR Mastermix.

1) Real-time PCR using $\mathrm{iQ}^{\mathrm{TM}} \mathrm{SYBR}^{\circledR}$ Green Supermix:

The mixture contains the hot-start enzyme, iTaq ${ }^{\mathrm{TM}}$ DNA polymerase. This enzyme is activated after an initial two minutes denaturation step at $95^{\circ} \mathrm{C}$. 
The master mix was prepared as follows:

$\begin{array}{ll}\text { Component } & \text { For } 1 \text { reaction } \\ \text { iQ SYBR Green Supermix } & 12.5 \mu \mathrm{l} \\ \text { Primer 1 (10uM) } & 0.75 \mu \mathrm{l} \\ \text { Primer 2 (10uM) } & 0.75 \mu \mathrm{l} \\ \text { Sterile Water } & 10 \mu \mathrm{l}\end{array}$

The master mix was aliquoted into the 96 well qPCR plate $(24 \mu \mathrm{l}$ per well) and $1 \mu \mathrm{l}$ cDNA was added per well. The plate was sealed, vortexed, centrifuged and loaded in the real-time PCR machine. One example of PCR was performed as follows:

\begin{tabular}{|l|l|l|l|l|}
\hline & \multicolumn{4}{|c|}{ Cycles(40 cycles) } \\
\hline Step & Taq activation & DNA Melting & Annealing & Extension \\
\hline Time & $2 \mathrm{~min}$ & $15 \mathrm{sec}$ & $30 \mathrm{sec}$ & $45 \mathrm{sec}$ \\
\hline Temp & $95^{\circ} \mathrm{C}$ & $95^{\circ} \mathrm{C}$ & $58^{\circ} \mathrm{C}$ & $72^{\circ} \mathrm{C}$ \\
\hline
\end{tabular}

The annealing temperature during PCR depends on the melting temperature of the primers used. The fluorescent signal was read after each extension step. After PCR, the melting curve of PCR product was recorded by slowly increasing temperature from $45^{\circ} \mathrm{C}$ to $95{ }^{\circ} \mathrm{C}$.

2) Real-time PCR using home-made PCR Mastermix:

The Home-made $10 \times$ PCR buffer was prepared as follows:

$\begin{array}{lll}\text { Component } & \text { Stock conc. } & \text { for } 10 \mathrm{ml} \\ \text { Tris-HCl }(\mathrm{pH} 8.8) & 1.5 \mathrm{M} & 5 \mathrm{ml} \\ \text { (NH4)2SO4 } & 1 \mathrm{M} & 2 \mathrm{ml} \\ \text { Tween-20 } & 10 \% & 100 \mu \mathrm{l} \\ \mathrm{H} 2 \mathrm{O} & & 2.9 \mathrm{ml}\end{array}$


Home-made PCR Mastermix was prepared as follows:

$\begin{array}{lll}\text { Component } & \text { Stock conc. } & \mu 1 \text { for 1 sample } \\ 10 \mathrm{x} \text { buffer } & 10 \mathrm{x} & 2.5 \\ \mathrm{MgCl} 2 & 25 \mathrm{mM} & 3 \\ \text { SYBR Green } & 1: 100 & 0.0313 \\ \text { dNTPs } & 20 \mathrm{mM} & 0.25 \\ \text { Taq } & 5 \mathrm{U} / \mu \mathrm{l} & 0.1 \\ \text { Triton X } 100 & 10 \% & 0.625 \\ \text { Trehalose (in } 10 \mathrm{mM} \text { Tris, } \mathrm{pH} 8.0) & 1 \mathrm{M} & 7.5\end{array}$

$1 \mathrm{M}$ Trehalose is dissolved in $10 \mathrm{mM}$ Tris $(\mathrm{pH} 8.0)$.

Reaction Set Up:

$\begin{array}{ll}\text { Component } & \text { For 1 reaction } \\ \text { Home-made } 10 \mathrm{x} \text { PCR Mastermix } & 14 \mu \mathrm{l} \\ \text { Primer 1 (10uM) } & 1.5 \mu \mathrm{l} \\ \text { Primer 2 (10uM) } & 1.5 \mu \mathrm{l} \\ \text { Sterile Water } & 7 \mu \mathrm{l} \\ \text { cDNA } & 1 \mu \mathrm{l}\end{array}$

One example of PCR was performed as follows:

\begin{tabular}{|l|l|l|l|l|}
\hline & \multicolumn{3}{|c|}{ Cycles(40 cycles) } \\
\hline Step & Taq activation & DNA Melting & Annealing & Extension \\
\hline Time & $2 \mathrm{~min}$ & $15 \mathrm{sec}$ & $30 \mathrm{sec}$ & $45 \mathrm{sec}$ \\
\hline Temp & $95^{\circ} \mathrm{C}$ & $95^{\circ} \mathrm{C}$ & $58^{\circ} \mathrm{C}$ & $72{ }^{\circ} \mathrm{C}$ \\
\hline
\end{tabular}

The annealing temperature during PCR depends on the melting temperature of the primers used. The fluorescent signal was read after each extension step. After PCR, the melting curve of PCR product was recorded by slowly increasing temperature from $45^{\circ} \mathrm{C}$ to $95{ }^{\circ} \mathrm{C}$. 
In the Real-time PCR, the following primers were used:

\begin{tabular}{|l|l|l|}
\hline \multirow{2}{*}{ Primers } & \multicolumn{2}{l|}{ Sequence } \\
\hline \multirow{2}{*}{ P53 } & forward & 5'-ATGGAGGAGCCGCAGTCAGATC \\
\cline { 2 - 3 } & reverse & 5'-GGGAGCAGCCTCTGGCATTCTG \\
\hline \multirow{2}{*}{ P21 } & forward & 5'-TAGGCGGTTGAATGAGAGG \\
\cline { 2 - 3 } & reverse & 5'-AAGTGGGGAGGAGGAAGTAG \\
\hline \multirow{2}{*}{ PUMA } & forward & 5'-TCAGGATTCAGTTTCAGATCAG \\
\cline { 2 - 3 } & reverse & 5'-CTATTGGAAATGCACTTCATGC \\
\cline { 2 - 3 } & reverse & 5'-CAGGCACCTAATTGGGCTC \\
\hline \multirow{2}{*}{ P14ARF } & forward & 5'-TACTGAGGAGCCAGCGTCTA \\
\cline { 2 - 3 } & reverse & 5'-ACGGGTCGGGTGAGAGTG \\
\hline \multirow{2}{*}{ GAPDH } & forward & 5'-GAAGGTCGGAGTCAACGGATTTG \\
\cline { 2 - 3 } & reverse & 5'-CAGAGATGATGACCCTTTTGGCTC \\
\hline
\end{tabular}

\subsubsection{TaqMan MicroRNA Assays}

The TaqMan McroRNA Assays (Applied Biosystems) are designed to detect and quantify mature microRNAs (miRNAs). TaqMan MicroRNA Assays are done in a two-step reaction. First, cDNA is reverse transcribed from total RNA samples using miRNA-specific primers. Then, PCR products are amplified from cDNA samples. PCR products are detected using TaqMan probes. 
RT Set Up

Component

1 reaction

$100 \mathrm{mM}$ dNTP

$0.15 \mu \mathrm{l}$

Multiscribe Reverse Transcriptase, $50 \mathrm{U} / \mu \mathrm{l} \quad 1 \mu \mathrm{l}$

$10 \times$ RT buffer

$1.5 \mu 1$

RNase inhibitor

$0,188 \mu 1$

Nuclease-free water

$4,162 \mu 1$

5 x TaqMan MicroRNA RT primer

$3 \mu \mathrm{l}$

Total RNA sample (10 ng)

$5 \mu 1$

Total

$15 \mu 1$

The reaction mix was incubated at $16{ }^{\circ} \mathrm{C}$ for $30 \mathrm{~min}, 42{ }^{\circ} \mathrm{C}$ for $30 \mathrm{~min}, 85{ }^{\circ} \mathrm{C}$ for $5 \mathrm{~min}$ and then held at $4{ }^{\circ} \mathrm{C}$. Synthesized cDNA was stored at $-20{ }^{\circ} \mathrm{C}$.

PCR Set Up

Component

TaqMan 2 x Universal PCR Master Mix

Nuclease-free water

TaqMan MicroRNA Assays 20 x TaqMan Assay $1 \mu \mathrm{l}$

RT product

Total
$1.33 \mu 1$

1 reaction

$10 \mu 1$

$7.67 \mu 1$

$20 \mu 1$

Thermal Cycling Conditions

\begin{tabular}{|l|l|l|l|}
\hline & \multicolumn{3}{|l|}{ Cycles $(40$ cycles $)$} \\
\hline Step & Taq activation & Denature & Anneal/Extend \\
\hline Time & $10 \mathrm{~min}$ & $15 \mathrm{sec}$ & $60 \mathrm{sec}$ \\
\hline Temp & $95^{\circ} \mathrm{C}$ & $95^{\circ} \mathrm{C}$ & $60^{\circ} \mathrm{C}$ \\
\hline
\end{tabular}




\subsection{Biochemistry and Immunological methods}

\subsubsection{Immunofluorescence}

Prior to immunofluorescence, 7.000 cells were seeded in the well of a 96-well plate. One day later, cells were transfected with plasmids. 48 hours post-transfection, cells were fixed with $4 \%$ formaldehyde in PBS++ for $20 \mathrm{~min}$. After fixation, cells were washed twice with PBS++ and permeabilized with $0.5 \%$ Triton X-100 in PBS ++ for 10 min. Cells were washed twice for 5 min with PBS++ and incubated in blocking solution (10\% FCS) for 10 min. First antibody diluted in blocking solution was added to the well and incubated for 1 hour. After washing three times with PBS++ $(2 \mathrm{x}$ shortly and once for $5 \mathrm{~min})$, secondary antibody and Hoechst $(0.2$ $\mu 1 / \mathrm{ml}$, final conc.) diluted in blocking solution were added to the well and incubated for $45 \mathrm{~min}$. After incubation, cells were washed three times with PBS++ (2x shortly and once for $5 \mathrm{~min})$. All the incubation steps were done at room temperature.

The fluorescent pictures were taken by the BD Pathway system.

\subsubsection{Immunoprecipitation}

Adherent cells were scraped, and centrifuged at $4{ }^{\circ} \mathrm{C}(10 \mathrm{~min}$ at $3000 \mathrm{rpm})$. Cell pellet was resuspended in $1 \mathrm{ml}$ pre-cooled IP lysis buffer, and homogenized by pushing it 5 times through a $26 \mathrm{G}$ insulin syringe. Cell lysate was centrifuged at $4{ }^{\circ} \mathrm{C}(15 \mathrm{~min}$ at $14.000 \mathrm{rpm})$ to get rid of cell debris. Supernatant was transferred into a new tube, mixed with $50 \mu$ pre-equilibrated Protein-G-Sepharose (PGS) and incubated at $4{ }^{\circ} \mathrm{C}$ for 1 hour on a rotator. Protein/sepharose mixture was centrifuged at $4{ }^{\circ} \mathrm{C}(4 \mathrm{~min}$ at $14.000 \mathrm{rpm})$, and the supernatant was transferred to a new tube. $30 \mu \mathrm{l}$ of the lysate was taken as an input control, mixed with $30 \mu 16 \mathrm{x}$ Laemmli, boiled for $5 \mathrm{~min}$ at $95{ }^{\circ} \mathrm{C}$, and stored at $-20^{\circ} \mathrm{C}$. The rest was divided into two parts, mixed with $1 \mu \mathrm{g}$ of each antibody (one specifc antibody and one control antibody) and incubated at $4{ }^{\circ} \mathrm{C}$ for 2 hours in a rotator. After centrifugation at $4{ }^{\circ} \mathrm{C}(15 \mathrm{~min}$ at $14.000 \mathrm{rpm})$, supernatant was transferred to a new tube, mixed with $15 \mu 1$ PGS and $35 \mu 1$ sepharose, incubated at $4{ }^{\circ} \mathrm{C}$ for 1 hour in a rotator and then centrifuged at $4{ }^{\circ} \mathrm{C}(2 \mathrm{~min}$ at $3.000 \mathrm{rpm})$. PGS pellet was washed with $800 \mu \mathrm{l}$ IP lysis buffer by inverting 15 times at $4{ }^{\circ} \mathrm{C}$. After centrifugation $(2 \mathrm{~min}$ at 3.000 rpm), PGS pellet was washed five times with $800 \mu \mathrm{l}$ IP buffer, and twice with $500 \mu \mathrm{l}$ IP buffer 
by inverting 15 times at $4{ }^{\circ} \mathrm{C}$. The PGS pellet was finally mixed with $50 \mu 16 \mathrm{x}$ Laemmli buffer, heated at $95{ }^{\circ} \mathrm{C}$ for $5 \mathrm{~min}$, and stored at $-20{ }^{\circ} \mathrm{C}$.

\subsubsection{Western blotting analysis}

\section{Cell harvesting and lysis}

Adherent cells were either scraped in the culture medium, centrifuged and then lysed in the lysis buffer, or directly lysed in the culture plates by applying the lysis buffer after removing medium and washing. Cell lysates were vigorously shaken for 15 min at $4{ }^{\circ} \mathrm{C}$, and centrifuged for $15 \mathrm{~min}$ at $4{ }^{\circ} \mathrm{C}$. Supernatant was transferred to a new tube. Protein concentrations were measured by $\mathrm{BCA}$ protein assay. Samples were stored at $-20{ }^{\circ} \mathrm{C}$. Before loading samples to SDS-PAGE gels, samples were mixed with $6 \mathrm{x}$ Laemmli buffer and incubated for $5 \mathrm{~min}$ at $95{ }^{\circ} \mathrm{C}$.

\section{SDS-PAGE electrophoresis}

SDS-PAGE is a method of separating proteins in a polyacrylamide gel according to their electrophoretic mobility.

Gels were prepared as follows:

$\begin{array}{llll}\text { Resolving gel }(10 \mathrm{ml}) & 10 \% & 12 \% & 15 \% \\ \text { H2O } & 4 \mathrm{ml} & 3.3 \mathrm{ml} & 2.3 \mathrm{ml} \\ \text { Acrylamide }(30 \%) & 3.3 \mathrm{ml} & 4 \mathrm{ml} & 5 \mathrm{ml} \\ 1.5 \mathrm{M} \text { Tris }(\mathrm{pH} 8.8) & 2.5 \mathrm{ml} & 2.5 \mathrm{ml} & 2.5 \mathrm{ml} \\ 10 \% \text { SDS } & 100 \mu \mathrm{l} & 100 \mu \mathrm{l} & 100 \mu \mathrm{l} \\ 10 \% \text { APS } & 100 \mu \mathrm{l} & 100 \mu \mathrm{l} & 100 \mu \mathrm{l} \\ \text { TEMED } & 4 \mu \mathrm{l} & 4 \mu \mathrm{l} & 4 \mu \mathrm{l}\end{array}$




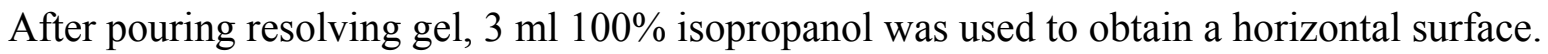

$\begin{array}{ll}\text { Stacking gel }(5 \mathrm{ml}) & 10 \% \\ \text { H2O } & 3.435 \mathrm{ml} \\ \text { Acrylamide }(30 \%) & 830 \mu \mathrm{l} \\ 1.5 \mathrm{M} \text { Tris }(\mathrm{pH} 8.8) & 630 \mu \mathrm{l} \\ 10 \% \text { SDS } & 50 \mu \mathrm{l} \\ 10 \% \text { APS } & 50 \mu \mathrm{l} \\ \text { TEMED } & 5 \mu \mathrm{l}\end{array}$

After polymerization, samples were loaded into the wells. Electrophoresis was performed at 80 $\mathrm{V}$ in $1 \mathrm{x}$ SDS-PAGE running buffer. When the protein samples passed the border between the two parts of the gel, higher voltage (ca.140 V) was applied.

\section{Western blot}

After desired separation was obtained, proteins were transferred onto a nitrocellulose membrane by western blot. Wet blot was performed in transfer buffer for $1.5 \mathrm{~h}$ at $100 \mathrm{~V}$ in a Biorad Chamber. The quality of transferring was checked by staining membranes with Ponceau S solution.

\section{Immunostaining}

After blotting, the membrane was blocked in 5\% milk dissolved in PBS-T or TBS-T (called "milk"). The membrane was then incubated with primary antibody diluted in milk for 2 hours or overnight $\left(4^{\circ} \mathrm{C}\right)$. Afterwards, the membrane was washed with PBS-T or TBS-T for three times for $7 \mathrm{~min}$, followed by incubation with secondary HRP-conjugated antibody for one hour. The membrane was washed again with PBS-T or TBS-T for three times for $7 \mathrm{~min}$. All washing and incubation steps were performed with gentle shaking. Finally, 1:1 mixture of Luminol/Enhancer solution and peroxide solution of SuperSignal West Dura or Femto (Pierce) was poured to the membrane, and the emitted light signal was detected by X-ray film. 


\subsubsection{Dual-luciferase reporter assay}

In our study, this assay was used to analyze microRNA function on predicted microRNA binding sites. The firefly luciferase reporter, which was used to evaluate microRNA function, contains 3'UTR sequences of predicted target genes. The Renilla (Renilla reniformis, also known as sea pansy) luciferase reporter was used for transfection normalization. In this assay, the activities of firefly and Renilla luciferases are measured sequentially from a single sample. The firefly luciferase reporter is measured first by adding Luciferase Assay Reagent II (LAR II) to generate a luminescent signal. After quantifying the firefly luminescence, this reaction is quenched, and the Renilla luciferase reaction is initiated simultaneously by adding Stop \& Glo® Reagent to the same sample.

\subsubsection{BCA protein assay}

This assay is based on the biuret reaction, in which $\mathrm{Cu}^{2+}$ was reduced to $\mathrm{Cu}^{+}$by protein in an alkaline medium. Bicinchoninic acid (BCA) then reacts with the reduced cation. The $\mathrm{BCA} /$ copper complex is water soluble and exhibits a strong absorbance at $562 \mathrm{~nm}$.

We use BCA protein assay kit from Pierce. First, a fresh set of BSA standards (diluted in the same solution as the samples) was prepared. Then, the BCA working reagent was prepared by mixing 50 parts of BCA Reagent A with 1 part of Reagent B. $5 \mu 1$ of the samples and BSA standards were mixed with $40 \mu 1$ working reagent, and incubated for $30 \mathrm{~min}$ at $37^{\circ} \mathrm{C}$. After incubation, samples and BSA standards were measured by using Nanodrop (Peqlab) BSA method. 


\section{Results}

\subsection{Characterization of p53 responsive microRNA-192/-215}

\subsection{1 p53 induces the expression of miR-192/-215}

In order to identify p53-responsive miRNAs, my colleague Christian Braun used a hybridization-based microarray to identify differentially expressed miRNAs in response to treatment with Nutlin-3 (a small molecule which inhibits Mdm2 and p53 interaction, resulting in p53 stabilization) in SJSA cells. In addition to miR-34a, a well-known p53 responsive miRNAs (Bommer, Gerin et al. 2007; Chang, Wentzel et al. 2007; Corney, Flesken-Nikitin et al. 2007; He, He et al. 2007; Raver-Shapira, Marciano et al. 2007; Tarasov, Jung et al. 2007; Tazawa, Tsuchiya et al. 2007), miR-194 was found to be induced by p53 (Braun, Zhang et al. 2008)(Fig. 4A). miR-194 can be expressed from two different miRNA clusters, namely the miR-194-1/215 cluster on chromosome 1 and the miR-194-2/192 cluster on chromosome 11. miR-192 and miR-215 from these two clusters are highly homologues to each other and share the same seed sequences. It seemed possible that miR-192/-215 is co-expressed with miR-194, although they were not detected above background fluorescence in our assay. Therefore, he further analyzed the expression of miR-192/-215 along with miR-194 in response to p53 by qRT-PCR, and all these miRNAs were robustly induced in SJSA cells (Fig. 4B). To confirm the responsiveness of miR-192 to genotoxic agents which normally activate p53, we treated A549 cells (human non-small cell lung adenocarcinoma cells, p53 wt) with camptothecin (CPT) and doxorubicin (DOX). miR-192 was induced by both agents (Fig. 4C), and the induction by camptothecin was abolished by siRNA to p53 (Fig. 4D). These results indicate that miR-192 is induced in a p53-dependent manner.

\subsubsection{Dysregulation of miR-192/-215 in colon cancers}

Previous studies showed that miR-192, -194 and -215 are highly expressed in colon and liver (Barad, Meiri et al. 2004; Lu, Getz et al. 2005). We checked the expression levels of miR-192 in a number of cell lines, and some but not all colon cancer-derived cell lines showed high expression (Fig. 5A). Interestingly, two colon cancer cell lines (HCT116 and LoVo) with wild 

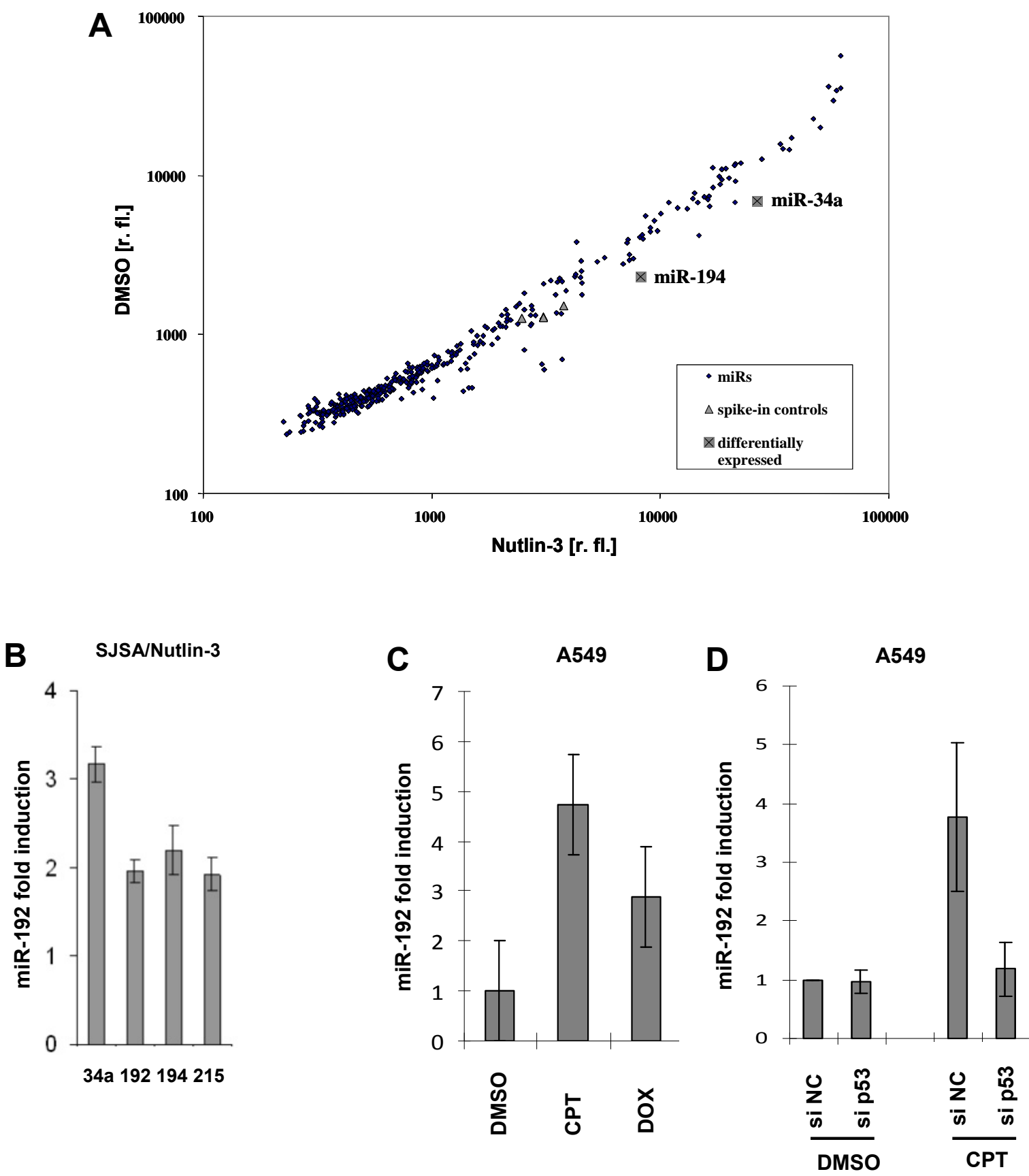

Figure 4. Induction of microRNAs through p53. A) microRNA expression profiling. RNA samples enriched for small RNA molecules, extracted from Nutlin-3 ( $8 \mu \mathrm{mol} / \mathrm{L})$ or DMSO treated SJSA cells, were labeled with $\mathrm{Cy} 3$ or $\mathrm{Cy} 5$ dyes and hybridized to three microarrays. The raw signal level for each probe is presented in relative fluorescence units (r.fl.), calculated as an average from three microarrays. Rhombi represent all analyzed microRNAs, triangles represent spiked-in synthetic control RNA molecules, and crossed squares represent microRNAs that showed a notable differential expression; B) qRT-PCR analysis of miR-34a, miR-192, miR-194 and miR-215 in SJSA cells treated with $8 \mu$ mol/L Nutlin-3 for $24 \mathrm{~h}$; C) qRT-PCR analysis of miR-192 in A549 cells treated with indicated drugs [camptothecin (CPT), $200 \mathrm{nmol} / \mathrm{L}$; doxorubicin (DOX), $100 \mathrm{ng} / \mathrm{mL}$ ] for $24 \mathrm{~h}$; D) qRT-PCR analysis of miR-192 in A549 cells. Cells were transfected with siRNAs. $24 \mathrm{~h}$ post-transfection, cells were treated with CPT $(200 \mathrm{nmol} / \mathrm{L})$ for another $24 \mathrm{~h}$. (Data from Fig. 4A \& B contributed by Christian Braun) 
type p53 express much lower levels of miR-192 compared to other colon cancer lines (HT29, DLD-1, HCC2998) with mutant p53. The comparatively low levels of miR-192 in HCT116 cells were not further affected by a deletion of the p53 gene (Fig. 5B). Additionally, the lung cancer cell line A549 (p53 wt) showed high levels of miR-192 (Fig. 5A). About $40 \%$ to $50 \%$ of colon cancers have p53 mutations (Iacopetta 2003), and we speculated that miRNAs under study may be down-regulated in a subset of colon cancers compared to normal mucosa. Thus, we compared the expression of miR-192/-215/-194 in 49 stage II colon cancers with that in 10 normal mucosa by using spotted LNA-based microarrays. Indeed, all three miRNAs were down-regulated in colon cancers relative to normal mucosa (Fig. 5C). The down-regulation was more pronounced in cancers with MSS (microsatellite stable) relative to cancers with MSI (unstable microsatellite). Interestingly, p53 mutations are more common in MSS tumors than in MSI tumors (Kim, Colangelo et al. 2007). However, because the samples did not allow simultaneously detection of p53 mutations, we did not know to what extent this dysregulation is due to the p53 status. Considering the dysregulation of miR-192/-215 in colon tumors, we further speculated that miR-192/-215 might have tumor suppressive activities.

\subsection{3 miR-192 suppresses cell clone formation in a partially p53-independent}

\section{manner}

In line with miR-34a which acts as a p53 effector, miR-192, as another p53 responsive miRNA, might also function as a tumor suppressor. In order to confirm this hypothesis, we performed colony formation assays in several cell lines. U2OS cells, expressing wild type p53, were transfected with expression vectors for miR-192 or miR-34a (Voorhoeve et al., 2006), followed by selection of stable transfectants with blasticidine. miR-192 caused remarkable reduction of cell colonies, to an extent comparable with miR-34a (Fig. 6A). This clonogenic assay was also carried out in H1299 cells, a cell line that is p53 null. miR-192 still suppressed colony formation as efficiently as miR-34a (Fig. 6B). These results suggest that both miRNAs can inhibit cell growth by mechanisms which are at least partially independent of p53. 
A

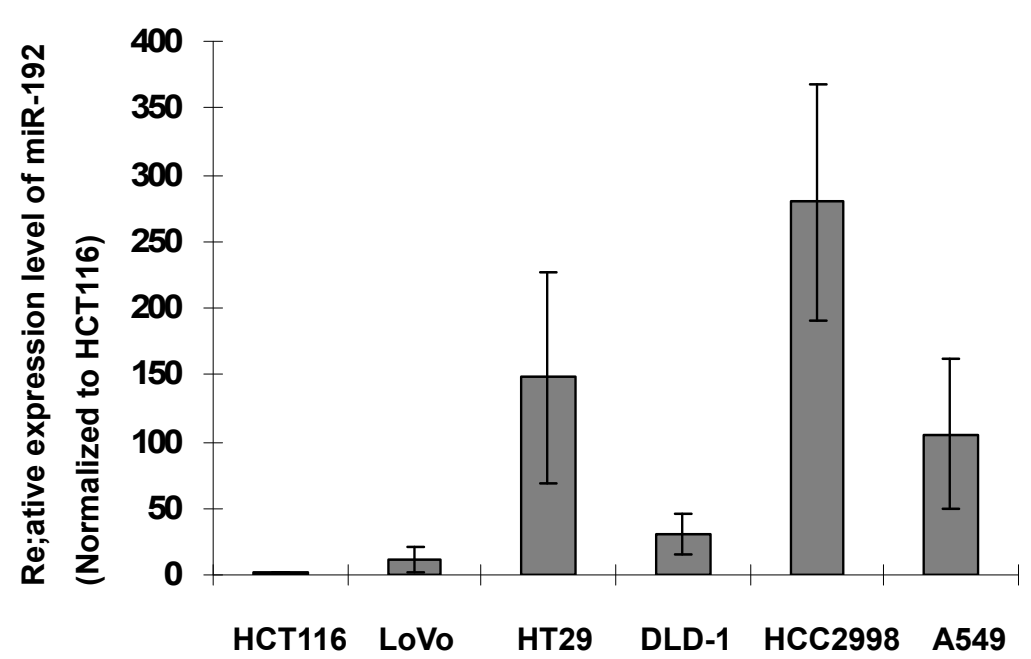

HCT116 LoVo HT29 DLD-1 HCC2998 A549
B

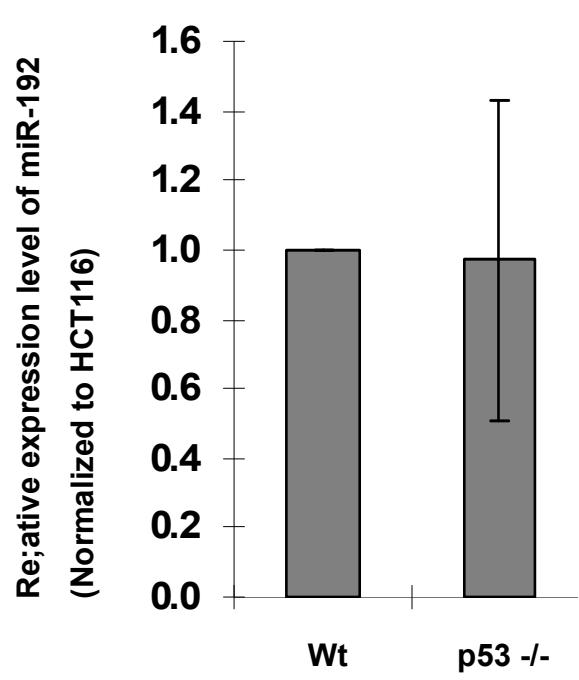

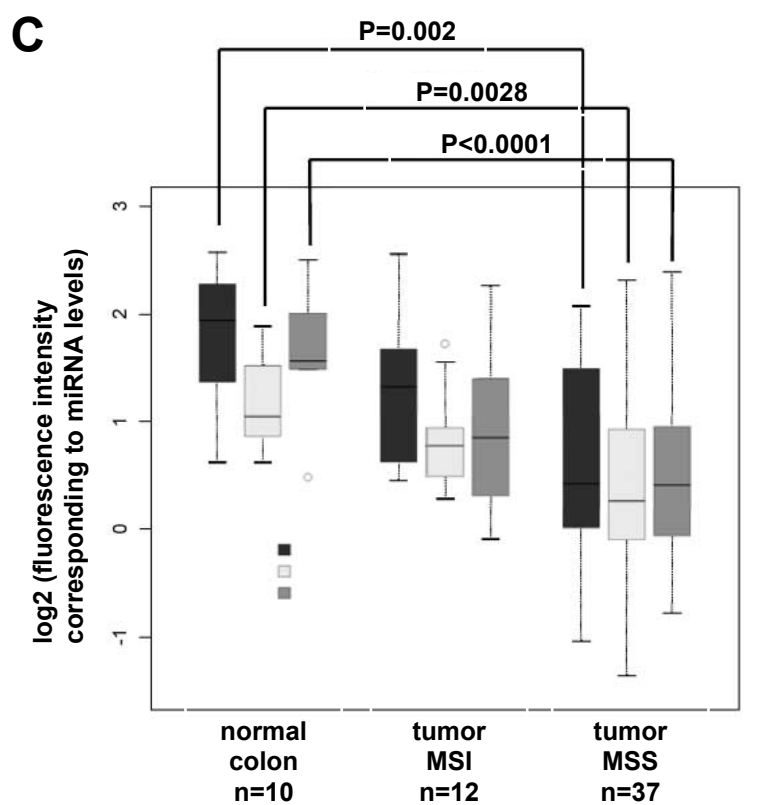

Figure 5. miR-192 levels in colon cancers. A) qRT-PCR analysis of miR-192 levels in different colon cancer cell lines (HCT116, LoVo, HT29, DLD-1 and HCC2998) and also the lung adenocarcinoma cell line A549; B) qRT-PCR analysis of miR-192 levels in HCT116 wt and p53 -/- cells; C) miRNA expression levels from colon cancers and normal colon mucosa determined by array hybridization. The $\log 2$ of hybridization intensities were determined for each sample, grouped according to its origin: normal colon mucosa, colon caner with MSI ,and colone cancer with MSS. The data from this study are summarized in a box plot format, including the level of significance that distinguishes the MSS tumors from normal tissue for each microRNA. 
A U2OS

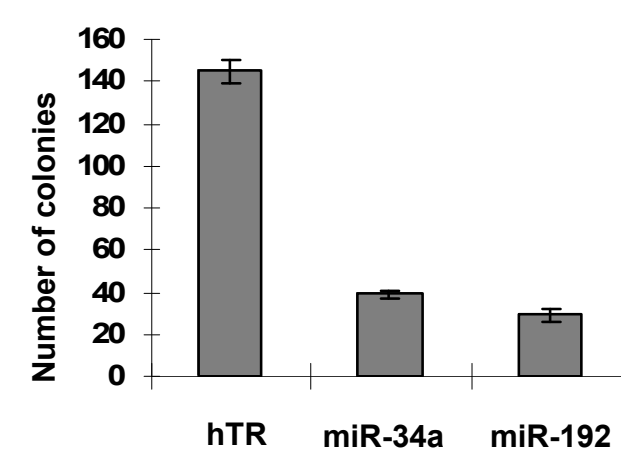

B $\quad \mathbf{H 1 2 9 9}$

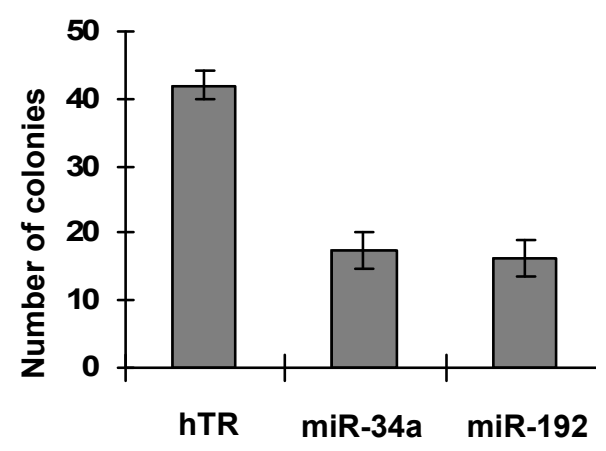

Figure 6. Colony formation assay. U2OS (A) or H1299 (B) cells were transfected with control miR-vector (hTR) or miR-vec-34a or miR-vec-192. The cultures were maintained for 2 weeks with blasticidin $(5 \mu \mathrm{g} / \mathrm{mL})$ to selecte stable transfectants, and the cells were then fixed, stained with crystal violet, and photographed from three independent experiments.

\subsection{4 microRNA-192/-215 provides a positive feedback to the p53 pathway}

Although miR-34a is a well known p53 effector, it also functions as a p53 regulator (Tazawa et al., 2007). Thus, we asked whether the newly identified p53-responsive miRNAs feed back to the p53 pathway. This may partially explain why miR-192 exhibits tumor suppressive activity. To test this, SJSA and HCT116 cells, both expressing wild type p53, were transfected with miR-192, miR-194, miR-215 and miR-34a individually. Then we detected p53 and its target gene product p21 using immunoblot analysis. Indeed, ectopic miR-192 and miR-215 up-regulated p53 and p21 levels in both cell lines, whereas miR-34a and miR-194 had no effects in SJSA cells and did so only to a lesser extent in HCT116 cells (Fig. 7A). In HCT116 cells with wild type p53, but not in their p53 -/- counterparts, miR192 increased both p21 mRNA and protein levels (Fig. 7B \& C), strongly suggesting that p21 activation by miR-192 is dependent on p53. Pro-apoptotic p53 target gene products (Puma and Noxa) accumulated less or not at all (Fig. 7B), suggesting that miR-192 mostly activates the cell cycle arrest function of p53 other than apoptosis. In order to clarify whether endogenous miR-192 can also modulate the p53 pathway, we antagonized miR-192 in A549 cells, which show high levels of miR-192 (Fig. 5A), by using antisense LNA oligonucleotides (and a scrambled control LNA), while inducing p53 with 5-fluorouracil (5-FU). 5-FU treatment accumulated both p53 and p21, 
but the induction was attenuated by knockdown of miR-192 (Fig. 7D). These observations indicate that miR-192 acts as an effector as well as a positive regulator of p53.

A SJSA

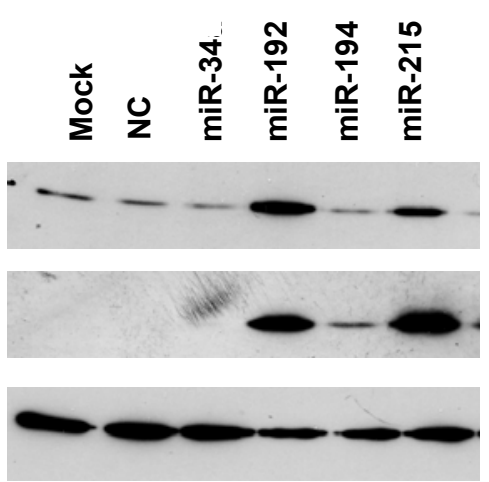

B

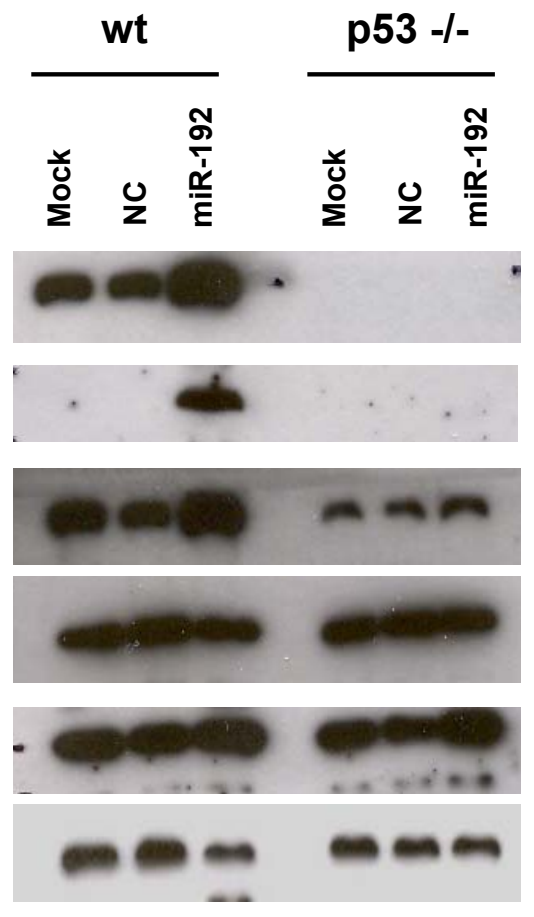

HCT116

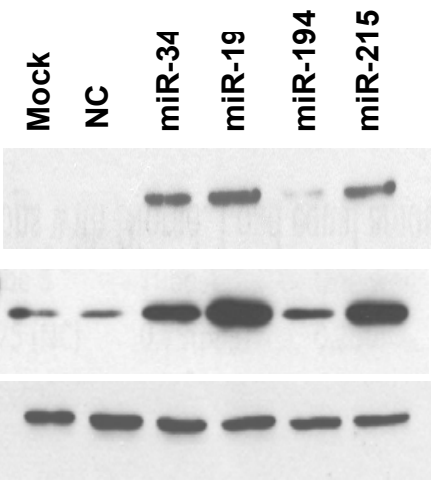

p53

p21

Beta actin

C HCT116

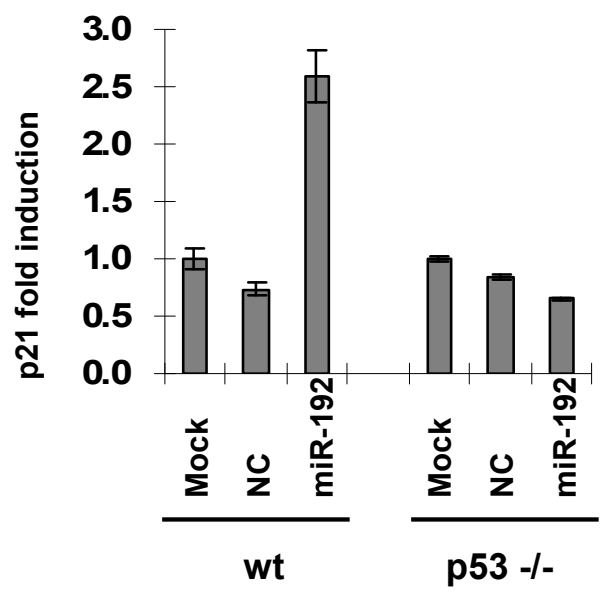

D $\quad$ A549

Puma

Noxa

Beta actin

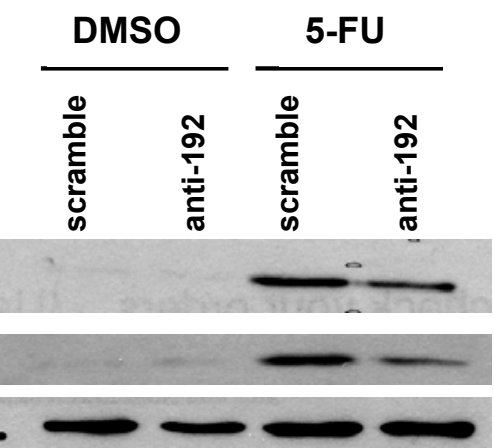

p53

p21

Beta actin

Figure 7. The effects of p53-responsive miRNAs on p53 pathway. A) SJSA or HCT116 cells were transfected with the indicated miRNAs $(10 \mathrm{nM})$ and incubated for $48 \mathrm{~h}$; B \& C) HCT116 wt or p53 -/- cells were treated as A followed by immunoblot (B) or qRT-PCR analysis (C); D) A549 cells were transfected with either scramble LNA or anti-miR-192 LNA $(100 \mathrm{nM})$ and then (24 post-transfection) treated with 500 $\mu \mathrm{mol} / \mathrm{L} 5$-FU or DMSO alone. After $24 \mathrm{~h}$ of 5-FU treatment, the cells were harvested and cell lysates were subjected to WB analysis. 


\subsection{5 miR-192/-215 is capable of inducing cell cycle arrest}

As suggested above, miR-192 may interfere with cell cycle progression by up-regulating p53 and p21. To test this, HCT116 cells were transfected with miR-192, miR-194, miR-215 and miR-34a, and cell cycle distribution was measured using a Nocodazole-trap assay. Briefly, cells were treated with Nocodazole 48 hours post transfection and monitored for cell cycle distribution 18 hours after Nocodazole treatment. Proliferating cells accumulated in G2 phase, as shown by mock and negative control transfected cells (Fig. 8A). But miR-192 and miR-215, as well as miR-34a, not miR-194, retained a portion of cell population in the G1 phase (Fig. $8 \mathrm{~A})$, suggesting that the cells were arrested in the G1 phase. This arrest was not observed in HCT116 cells lacking either p53 or p21 (Fig. 8A \& B). Therefore, miR-192 and miR-215 can arrest cells in G1 by mechanisms that are at least partially dependent on p53 and p21. My colleagues further confirmed that miR-192 and miR-215 were both capable of inducing a moderate but reproducible accumulation of HCT116 cells in G2/M (Braun et al., 2008), but not in HCT116 cells lacking p53. In contrast to miR-34a, miR-192 and miR-215 did not induce significant appearance of cells in a sub-G1 fraction (indicative of cell death) (Braun et al., 2008). In summary, miR-192 and miR-215 induce cell cycle arrest in G1 and G2/M rather than apoptosis, as demonstrated by miR-34a.

\subsubsection{Knockdown of miR-192 sensitizes A549 cells to 5-fluorouracil}

Having demonstrated that knockdown of miR-192 attenuated the accumulation of p53 and p21 in response to 5-fluorouracil (Fig. 7D), we asked what biological consequences it might cause. We used flow cytometry to analyze the distribution of DNA content, and 5-FU induced more cells in a sub-G1 fraction (indicative of cell death) when miR-192 was knockdown in A549 cells (Fig. 9). Perhaps it is because the antiapoptotic activity of p21 (Bunz et al., 1999; Hemmati et al., 2005; Wendt et al., 2006) was now compromised. 

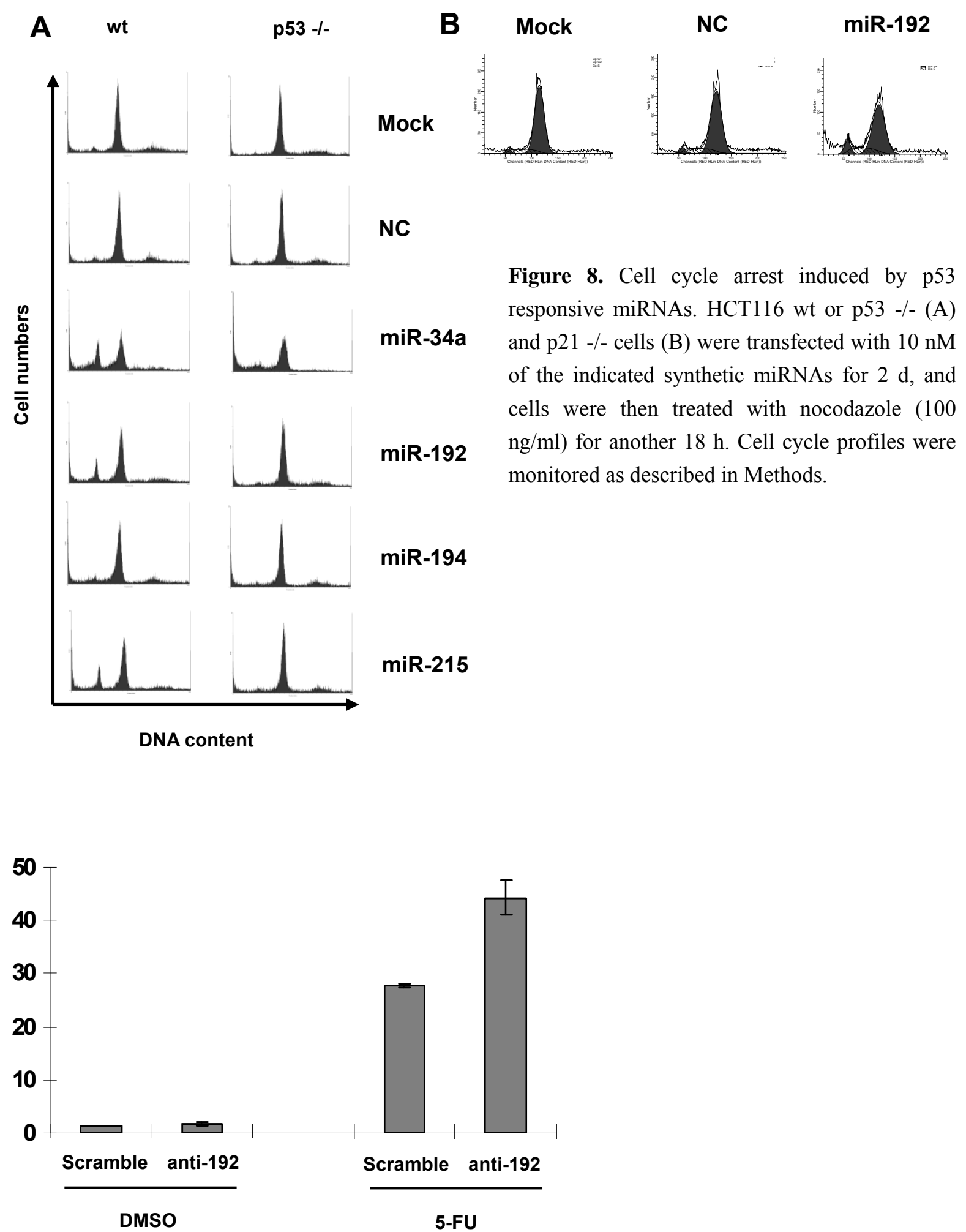

Figure 9. Knockdown of miR-192 sensitizes A549 cells to 5-fluorouracil. Cells were transfected with either scramble LNA or anti-miR-192 LNA (100nM) and then (24 post-transfection) treated with $500 \mu \mathrm{mol} / \mathrm{L} 5-\mathrm{FU}$ or DMSO alone. After $48 \mathrm{~h}$ of 5-FU treatment, the cells were then trypsinized, fixed with ethanol and stained with propidium iodide for flow cytometry analysis. 


\subsection{Identifying p53-regulatory microRNA-22}

\subsubsection{Immunofluorescence screening for p53 regulatory microRNAs}

p53 regulation occurs primarily through the mechanisms that affect the stabilization or degradation of the $\mathrm{p} 53$ protein. miRNAs may provide a novel layer of p53 regulation by interfering with the stability and/or translation of p53 mRNAs, or by targeting any of its regulatory components. Our previous study revealed that miR-192 and -215 are positive regulators of p53 activity and induce p53-dependent cell cycle arrest (Braun et al., 2008). In addition, miR-125b was identified to be a negative regulator of p53 and p53-induced apoptosis during development and during the stress response in zebrafish and human cells (Le et al., 2009). To perform a comprehensive analysis to identify miRNAs that regulate p53, we developed an immunofluorescence screening in U2OS cells. As shown in Fig. 10A, U2OS cells were transfected to express each miRNA separately in a 96 well format, using a miR-vector collection provided by Reuven Agami (Voorhoeve et al., 2006). A co-transfected GFP plasmid was used to identify successfully transfected cells. Cells were fixed and stained with p53 antibody, followed by a dye-coupled secondary antibody staining. Using an automated fluorescence microscope and analysis software (BD pathway), nuclear p53 fluorescence intensities, where DAPI staining was used to define the region, were determined in GFP-positive cells per well. The following box plot analysis provided a summary of the distribution of p53 intensities among cells per well (Fig. 10B). The median values from each well were extracted and calculated to obtain z-scores (Fig. 10C), which represent the distance between the median value of an individual well and the population mean in the units of standard deviation. The bigger the absolute value of the z-score is, the more significant is the effect on p53 levels. As shown in Fig. 10B and C, all positive controls (pCMV p53 plasmid expressing wild type p53 and p14arf plasmid inducing endogeous p53; whereas pSuper p53 plasmid expressing short hairpin RNA against p53) had pronounced effects, either up- or down-regulating $\mathrm{p} 53$, confirming the sensitivity of this screening method.

Using this screening method, we identified several candidates from the miR-vector collection affecting p53 levels. In addition to miR-125b, a known p53 negative regulator, we identified miR-22 which down-regulated p53 levels (Fig. 11A). In a parallel screening, 36 hrs 
post-transfection of miR vectors we treated the cells with $1 \mu \mathrm{M}$ camptothecin (CPT, a genotoxic agent) for $12 \mathrm{hrs}$, in order to identify miRNAs affecting p53 activation in response to DNA damage. Again, miR-22 had the most pronounced down-regulating effects on p53 levels (Fig. 11B). Together, this immunofluorescence screening identified miR-22 as another negative regulator of $\mathrm{p} 53$.

A

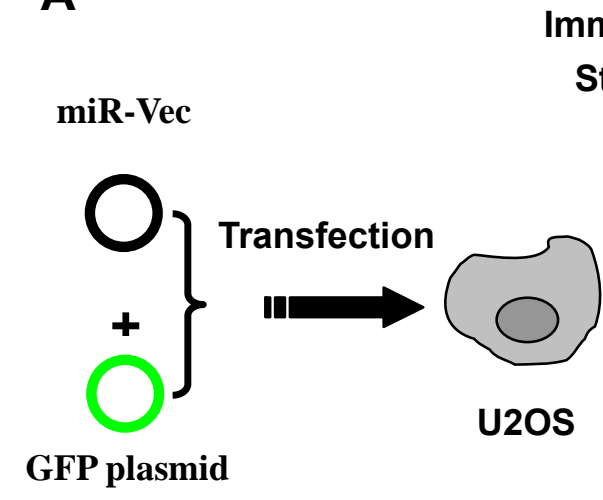

Immunofluorescence-

Staining (anti-p53)

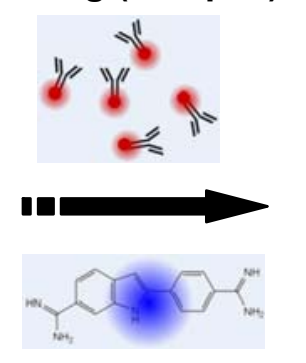

Nuclear Staining (DAPI)

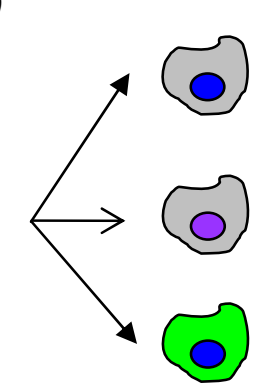

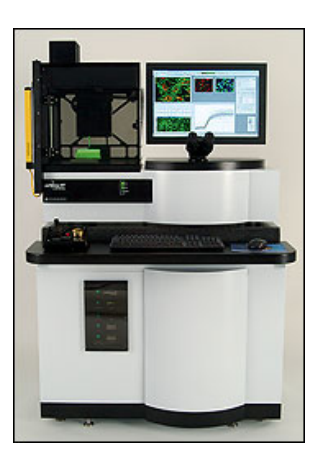

BD pathway

\section{B Plate2 (GFP $>=700$ )}

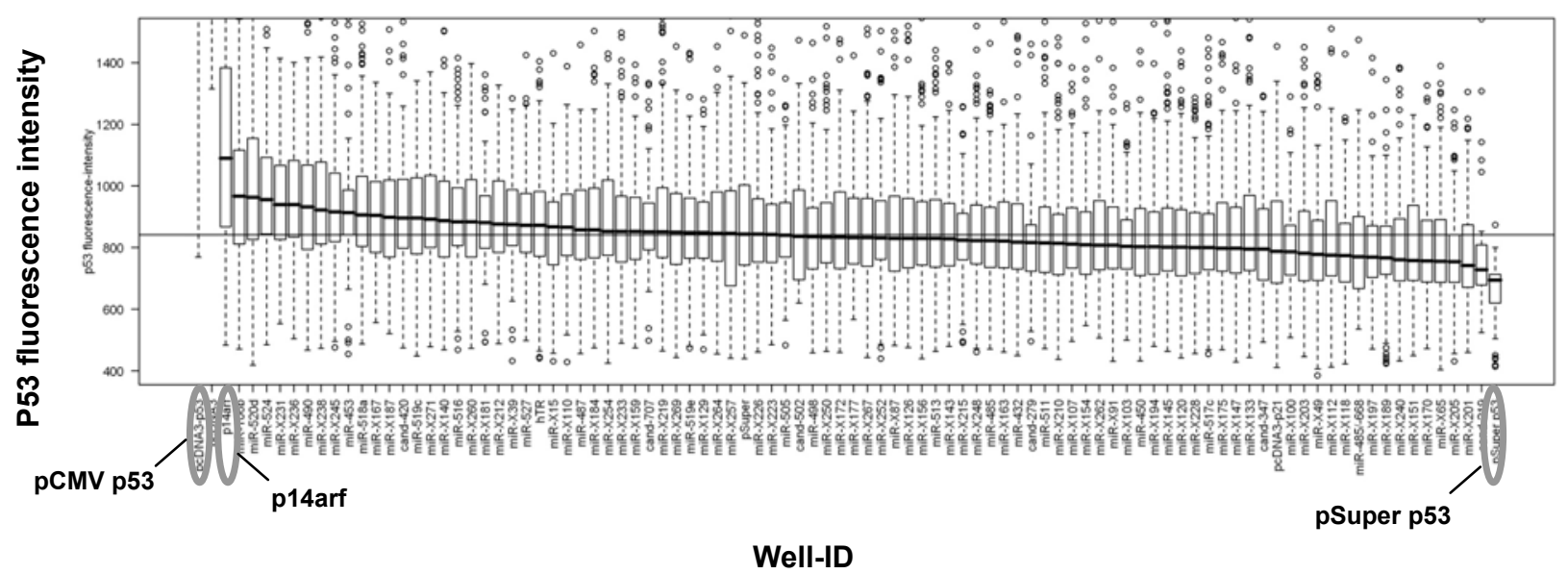

Well-ID

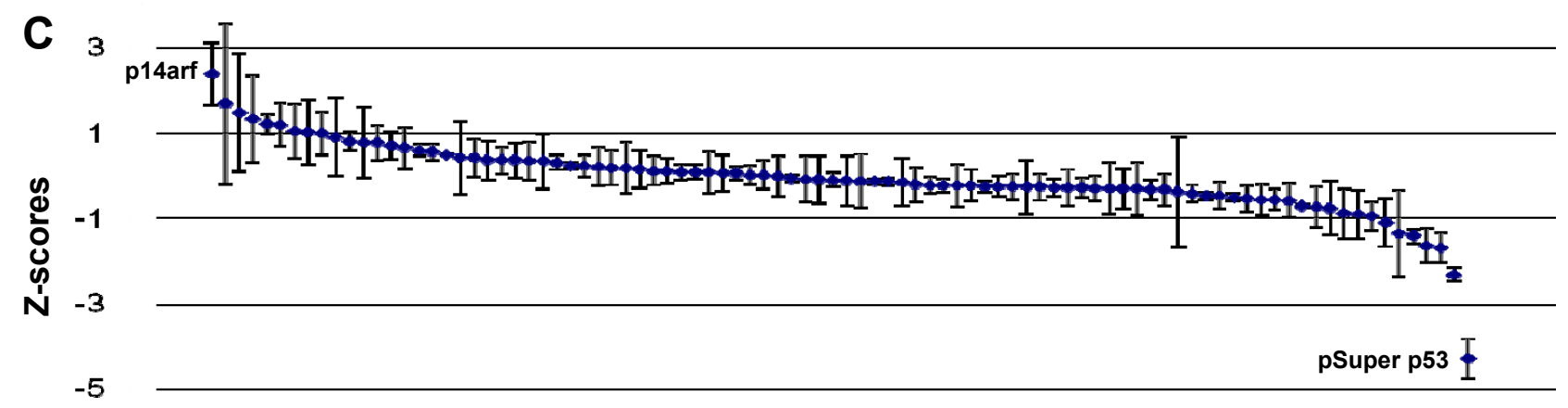


Figure 10. Set-up of an immunofluorescence screening for identifying p53 regulatory microRNAs. A) The scheme of immunofluorescence-based screening provided by Andreas Scheel (more details in the text); B) Box plot analysis. It provided a summary of the distribution of p53 intensities among cells per well. The wells were sorted according to the median values of p53 intensities in each well; C) Z-score analysis. The median values from each well were extracted and calculated to obtain $z$-scores. The wells were sorted according to the values of z-score obtained from each well. The error bar represents the variant from three independent experiments.
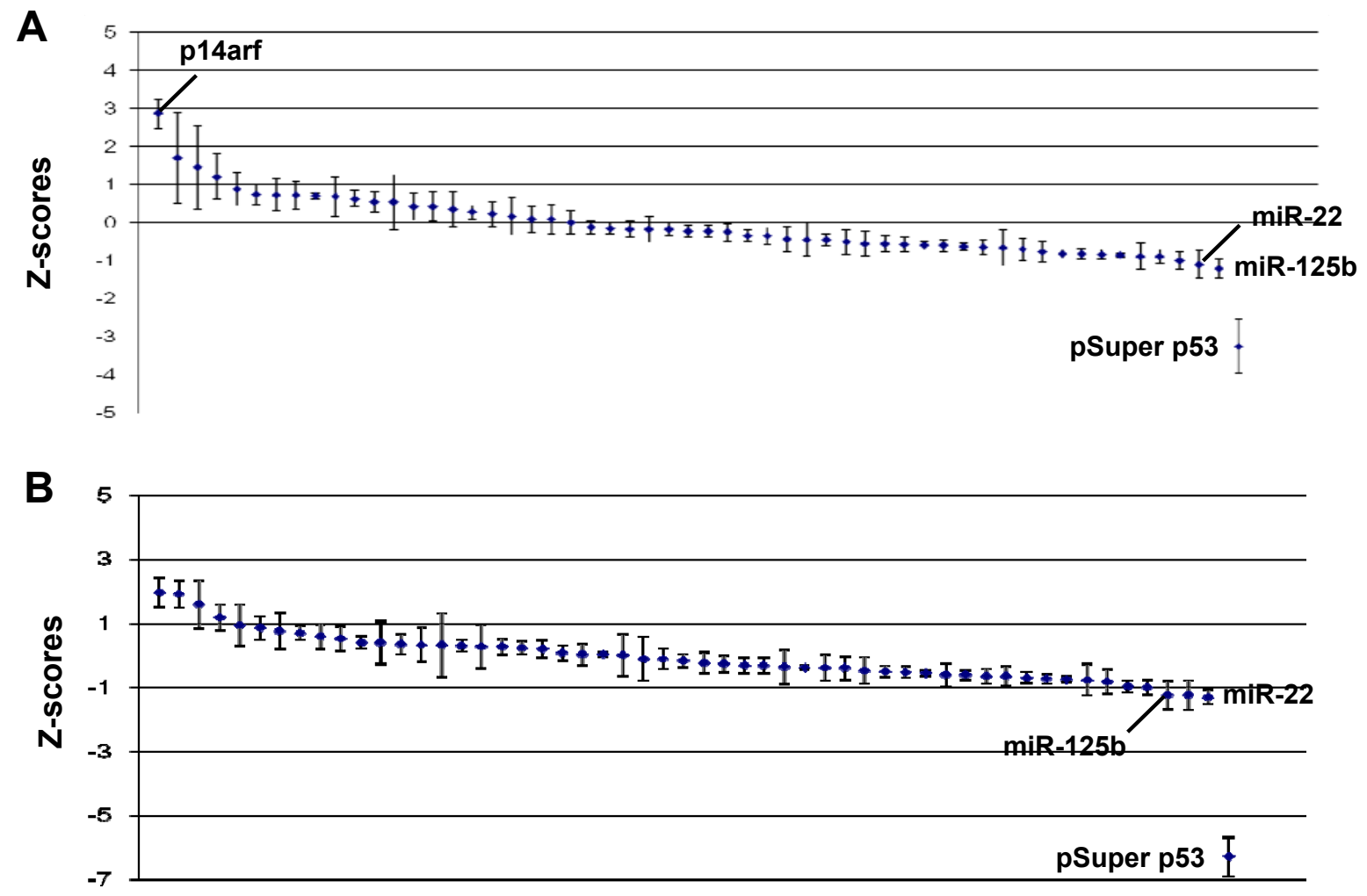

Figure 11. Identifying p53 regulatory microRNAs. The method has been described in the text and Figure 10. A) Z-score analysis of immunofluorescence-based screening. Three independent experiments were performed; B) In a parallel experiment $36 \mathrm{hrs}$ post-transfection, cells were treated with $1 \mu \mathrm{M} \mathrm{CPT} \mathrm{(a}$ genotoxic agent) for $12 \mathrm{hrs}$. Then cells were subjected to immunofluorescence and z-score analysis.

\subsection{2 miR-22 down-regulates p53 levels and activities}

To verify the results of the screening, we transfected U2OS cells with synthetic miR-22 (Ambion), followed by immunoblot and quantitative RT-PCR analysis. As shown in Fig. 12A, miR-22 down-regulated p53 levels. An even more profound effect on p21, a well established p53 target gene product, was observed. In addition to p21, the expression level of another p53 
target gene, Puma, was also repressed by miR-22 as revealed by quantitative RT-PCR (Fig. 12B). However, Mdm2 levels were not affected by miR-22 (Fig. 12A). The effects of miR-22 on the p53 pathway were also checked in another cell line HCT116 (Fig. 12C). Although p53 levels were almost not affected, p21 was profoundly down-regulated. Moreover, p21 was not affected by miR-22 in HCT116 p53 -/- cells (Fig. 12D), suggesting that p21 down-regulation by miR-22 was dependent on $\mathrm{p} 53$.

As shown from the screening, miR-22 also interferes with p53 activation in response to genotoxic agent (Fig. 11B). Indeed, immunoblot analysis clearly showed that ectopic expression of miR-22 repressed p53 and p21 levels in both U2OS and HCT116 cells treated with CPT (Fig. 13A). In response to DNA damage, p53 is normally activated via phosphorylation and subsequent acetylation. We hypothesized that the levels of phospho-p53 and/or acetyl-p53 are also affected. In line with this, phospho-p53 (Ser15) and acetyl-p53 (Lys382) were profoundly down-regulated by miR-22 (Fig. 13B), strongly suggesting that miR-22 represses p53 activities.

\subsection{3 miR-22 did not directly target $p 53$ and p21 mRNAs}

Next, we asked whether miR-22 directly target the p53 mRNA, preventing its translation and/or stability. By using TargetScan (Lewis et al., 2005) that predicts MicroRNA targets, we found that both p53 and p21 3'UTR have conserved binding sites for miR-22 (data not shown). To assess the binding of miR-22 to the 3'UTR of the p53 gene, we performed a Dual-luciferase reporter assay. We cloned the 3'UTR of p53 into the pMIR-REPORT Luciferase Reporter (Ambion). The firefly luciferase activity was then measured to assess the suppression effects of miR-22, and renilla luciferase activity was used for transfection normalization. Upon sequential transfections of synthetic miR-22 and these reporter constructs in H1299 cells, we found that miR-22 had no inhibitory effects on the expression of the reporter gene with p53 3'UTR (Fig. 14A). We also obtained a luciferase reporter containing p21 3'UTR sequence from SwitchGear. Again, no inhibitory effects by miR-22 were observed (Fig. 14B). Both results indicated that miR-22 does not directly target p53 and p21. 
A U2OS

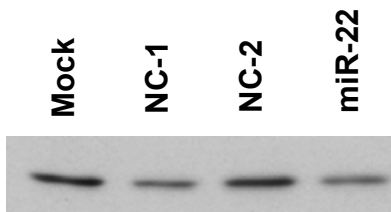

p53

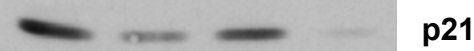

p21
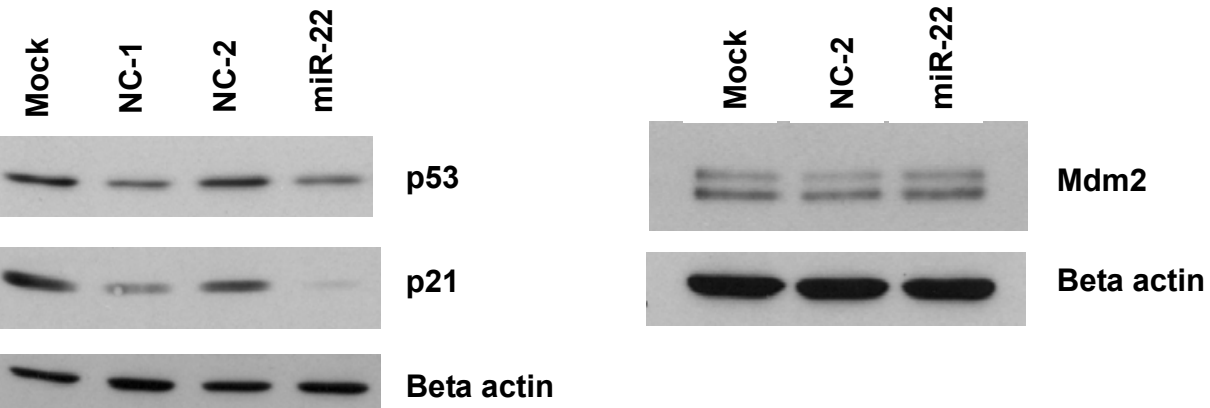

B U2OS
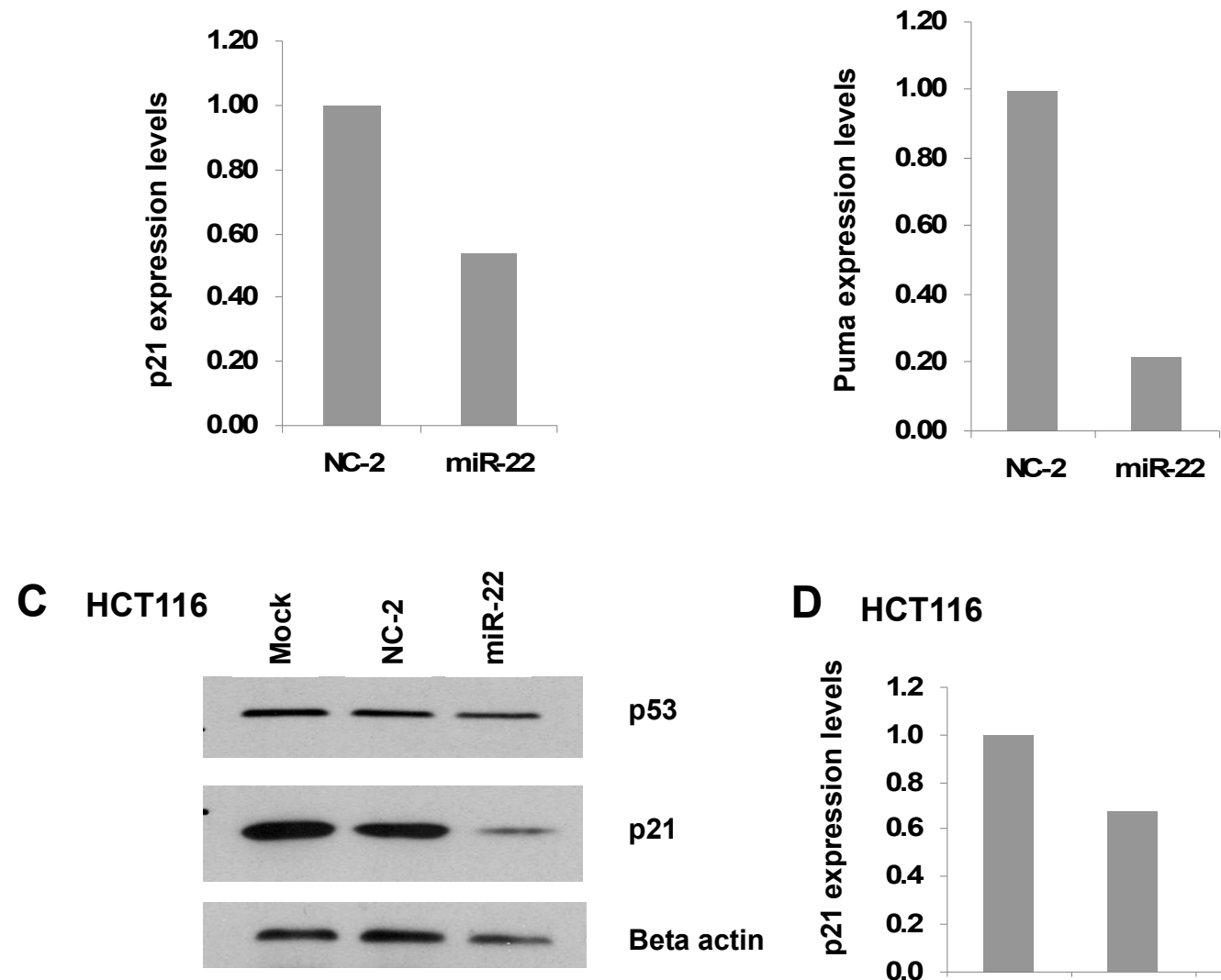

D HCT116

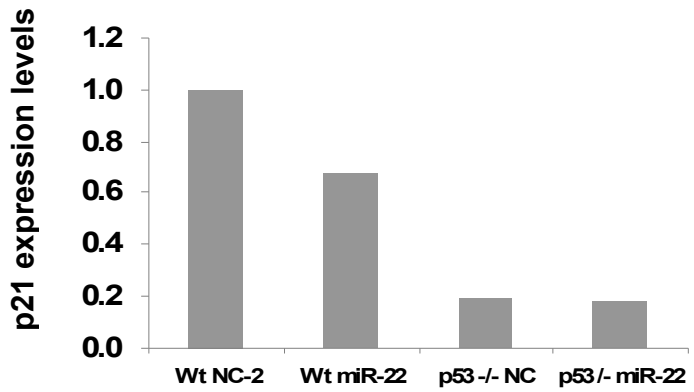

Figure 12. miR-22 down-regulates p53. A \& B) U2OS cells were transfected with the indicated miRNAs $(10 \mathrm{nM})$ and incubated for $48 \mathrm{hrs}$, followed by immunoblot (A) or qRT-PCR analysis (B); C) HCT116 cells were transfected with the indicated miRNAs $(10 \mathrm{nM})$ and incubated for $48 \mathrm{hrs}$; D) qRT-PCR analysis of p21 levels in miR-22 transfected HCT116 or their p53 -/- counterparts. Cells were treated as in C. 
A U2OS, CPT treated

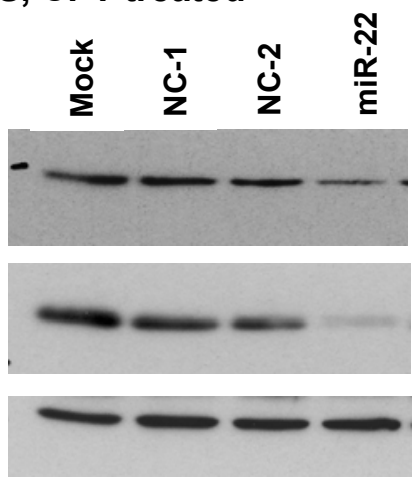

p21

Beta actin
HCT116, CPT treated

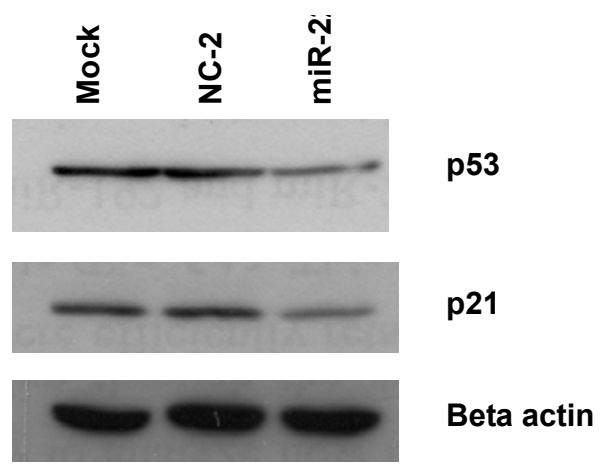

B U2OS, CPT treated

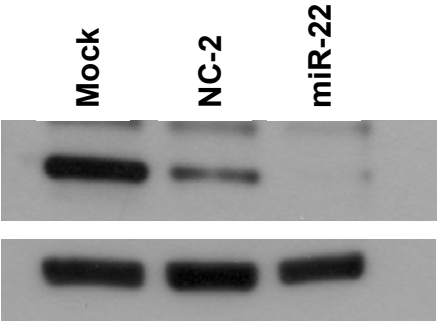

HCT116, CPT treated

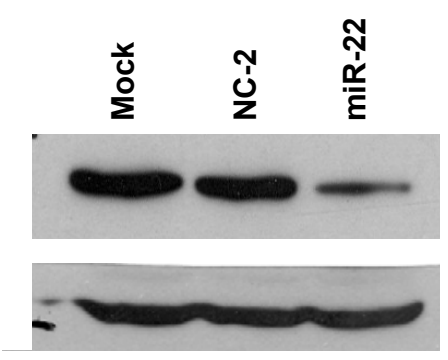

Phospho-p53

Beta actin

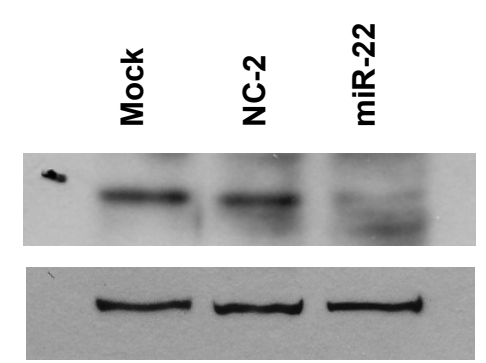

Acetyl-p53

Beta actin
Phospho-p53

Beta actin

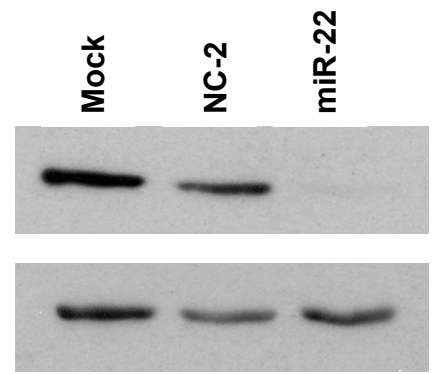

Acetyl-p53

Beta actin

Figure 13. miR-22 impaired p53 activation upon DNA damaging agent treatment. A) WB analysis of miR-22 transfected U2OS and HCT116 cells. Cells were transfected with the indicated miRNAs (10 nM). $36 \mathrm{hrs}$ post-transfection, cells were treated with $1 \mu \mathrm{M} \mathrm{CPT}$ for $12 \mathrm{hrs}$; B) WB analysis of miR-22 transfected U2OS and HCT116 cells. Cells were treated as A. Phospho-p53 (Ser15) and acetyl-p53 (Lys382) antibodies were used. 
A

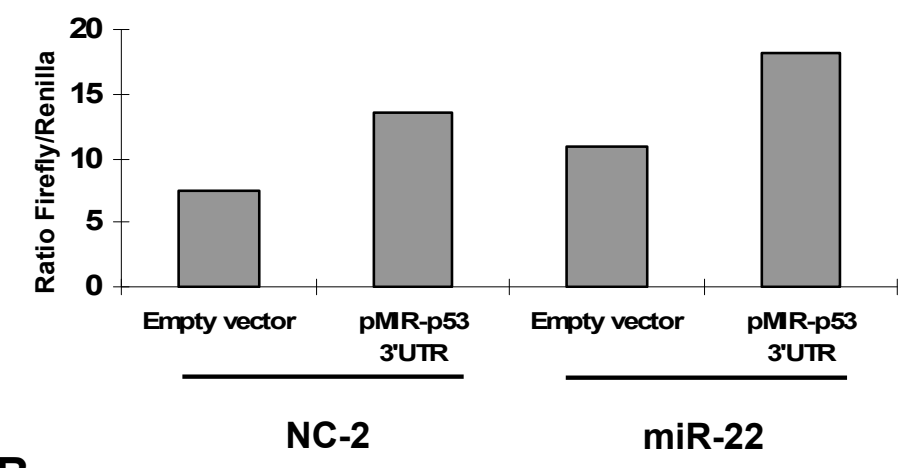

B

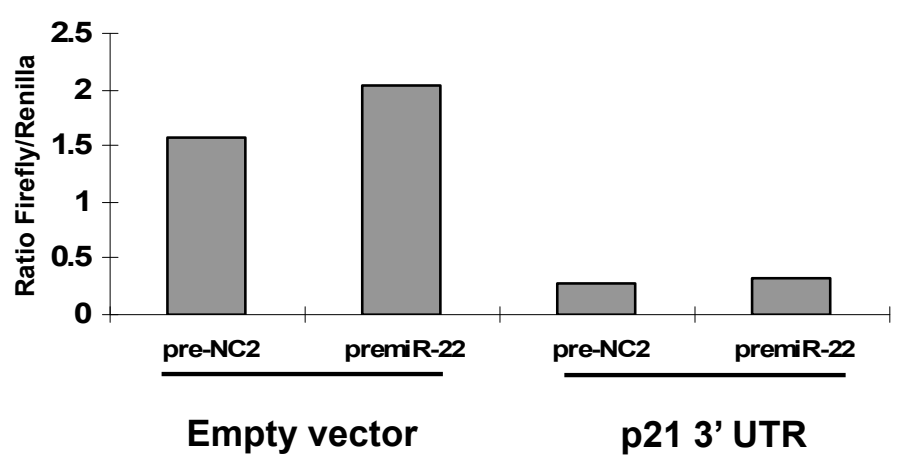

Figure 14. miR-22 does not directly target p53 and p21. A) H1299 cells were first reverse-transfected with miRNAs $(10 \mathrm{nM}) .24 \mathrm{hrs}$ post-transfection, cells were co-transfected with firefly luciferase repoter (which contains $p 53$ 3'UTR downstream of luciferase reporter gene or not) and renilla luciferase reporter and incubated for another 24 hrs. Cells were then harvested for dual luciferase assay (Promega); B) The method is the same as A, but firefly luciferase reporter contains $p 21$ 3'UTR downstream of luciferase reporter gene.

\subsection{4 miR-22 negatively regulates Sp1}

Interestingly, Sp1 mRNA was also predicted to be the target of miR-22 (data not shown). Sp1 is a p53 cofactor, which is required for the transcription of a number of p53-responsive genes, including p21 (Koutsodontis, Tentes et al. 2001). Overexpression of miR-22 down-regulated Sp1 levels in both U2OS and HCT116 cells (Fig. 15). It therefore appears that miR-22 reduces p53 activity not only by down-regulating the levels of p53 itself, but also by repressing its cofactor Sp1. However, the direct binding of miR-22 to Sp1 mRNA 3'UTR still needs to be assessed.

U2OS

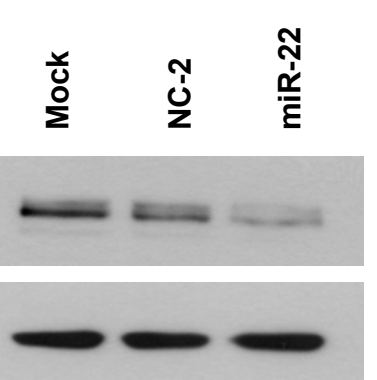

HCT116

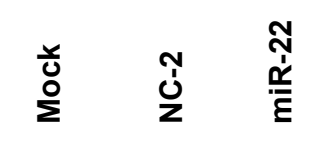

Sp1

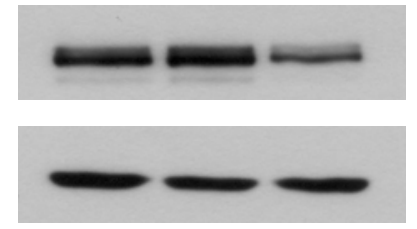

Beta actin

Figure 15. miR-22 negatively regulated Sp1. U2OS and HCT116 cells were transfected with the indicated miRNAs (10 $\mathrm{nM})$ and incubated for $48 \mathrm{hrs,} \mathrm{followed} \mathrm{by} \mathrm{immunoblot.}$ 


\subsection{5 miR-22 suppresses p53 activation by down-regulating Chk2}

In response to DNA damage, several protein kinases, including ATM/ATR, Chk1, Chk2, p38 and others, are involved in phosphorylating p53. We speculated that upstream kinases are affected by miR-22, leading to down-regulation of p53 phosphorylation as shown in Fig. 13B. We determined the levels of total Chk1 and phospho-Chk1 in miR-22 transfected cells, but they were not affected by miR-22 (Data not shown). But the levels of total Chk2 and phospho-Chk2 (Thr68) were significantly down-regulated by miR-22 (Fig. 16A), suggesting that the amount and activity of Chk2 were impaired. Chk2 phosphorylates the serine-20 in the transactivation domain of p53. In line with this, p53 phosphorylation at Ser20 was impaired by miR-22 (Fig. 16B). Thus, one mechanism of suppressing p53 activation by miR-22 is through down-regulating Chk2, although we don't know yet if miR-22 directly targets Chk2 or upstream ATM.

A

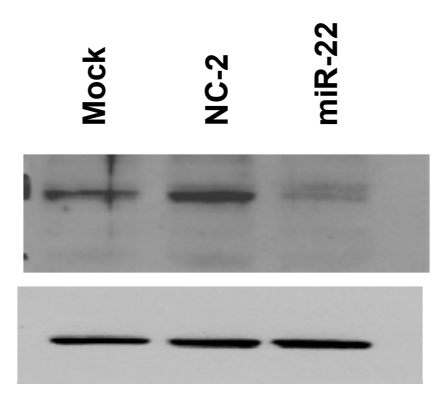

total Chk2
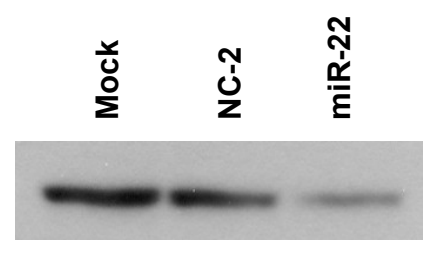

phospho-Chk2

hsc70

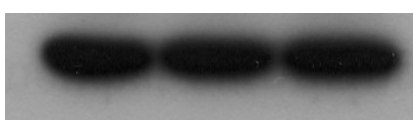

Beta actin

B

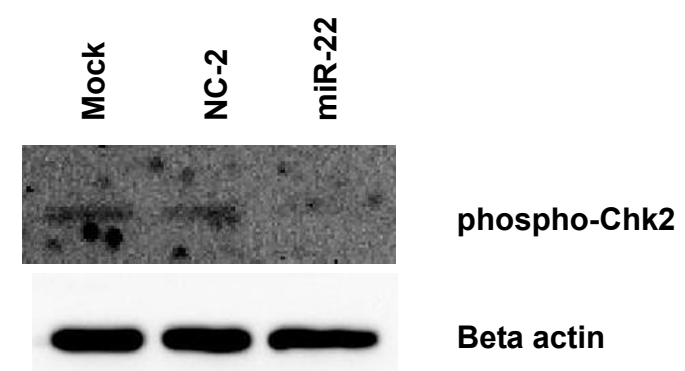

Figure 16. miR-22 downregulates Chk2 levels and activities. A) U2OS cells were transfected with the indicated miRNAs $(10 \mathrm{nM}) .36 \mathrm{hrs}$ post-transfection, cell were treated with CPT $(1 \mu \mathrm{M})$ and incubated for another 12 hrs. Cells were then subjected to immunoblot. The blots were stained with either total Chk2 antibody or phospho-Chk2; B) The same samples were subjected to immunoblot and the blot was stained with phospho-p53 (Ser20) antibody. (WB performed by Antje Dickmanns) 


\subsection{6 miR-22 induces DNA damage and is capable of inducing G2/M arrest}

As shown above, miR-22 negatively regulates p53 and p21. p21 plays important roles in G1/S checkpoint. Thus, we speculated whether the loss of p21 by miR-22 would prompt G1/S transition, leading to replicative stress and accumulated DNA damage. We transfected HCT116 cells with miR-22, followed by immunoblot analysis of $\gamma \mathrm{H} 2 \mathrm{AX}$ (a hallmark of DNA damage). Indeed, cells transfected with miR-22 had enhanced $\gamma \mathrm{H} 2 \mathrm{AX}$ (Fig. 17A), indicating DNA damage induced by miR-22. miR-22 also accumulated DNA damage in U2OS cells, although this was not as pronounced as in HCT116 cells (data not shown). However, we also observed DNA damage in HCT116 p53 -/- counterparts (Fig. 17A), suggesting this phenomenon is at least partially independent on $\mathrm{p} 53$.

We then monitored the cell cycle distribution after miR-22 transfection in U2OS using flow cytometry. miR-22 caused the accumulation of cells at G2/M (Fig. 17B). In line with this, cyclin B1 levels (G2/M phase marker) were higher in U2OS cells transfected with miR-22, as revealed by immunoblot (Fig. 17C). Histone $\mathrm{H} 3$ is specifically phosphorylated during mitosis so that phospho-Histone $\mathrm{H} 3$ is widely used as mitotic marker. As shown in Fig. 17D, cells treated with the microtubule disruption drug nocodazole, which induces mitotic arrest, accumulated phospho-Histone H3, but not miR-22 transfected cells, suggesting miR-22 might specifically induce G2-arrest.

\subsection{7 miR-22 suppresses cell clone formation}

Although miR-22 is a negative regulator of p53, it induces $\mathrm{G} 2 / \mathrm{M}$ arrest, suggesting it has tumor suppressive activities rather than functions as an oncogene. To confirm this, we performed colony formation assays in U2OS cells. Cells were transfected with the expression vectors for miR-22 (Voorhoeve et al., 2006), followed by selection of stable transfectants with blasticidine. miR-22 caused a remarkable reduction of cell colonies compared to the negative control (Fig. 18). It strongly indicated that miR-22 has anti-proliferative activities. 


\section{A HCT116}

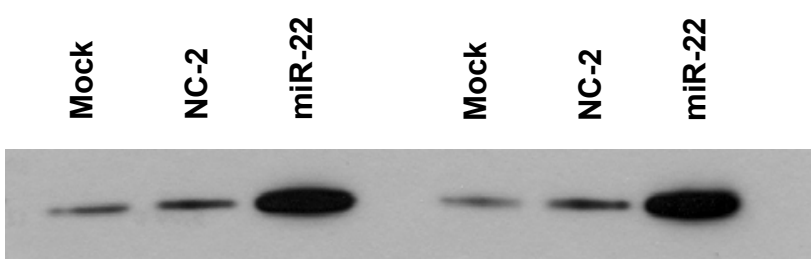

YH2AX

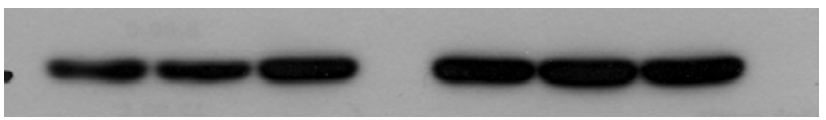

Beta actin

B U205

Mock

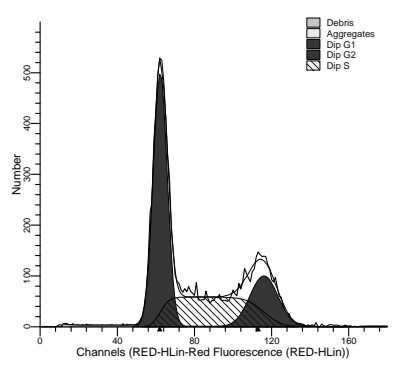

C U2Os

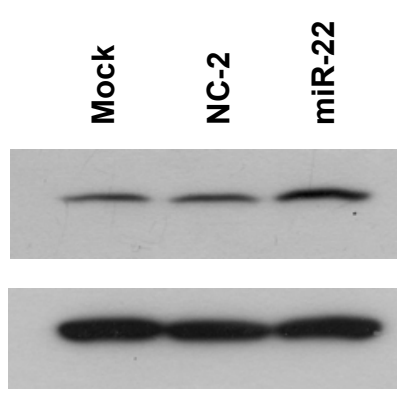

NC

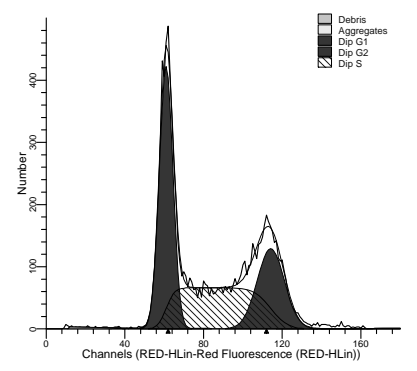

miR-22

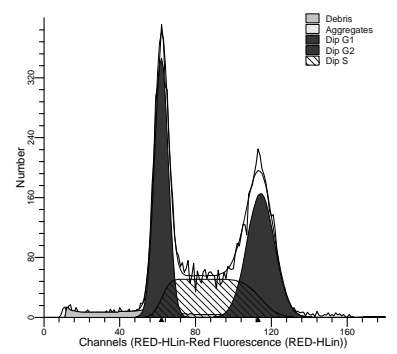

D U2OS

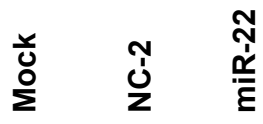

Cyclin B1

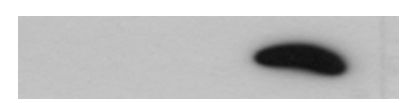

Phospho-Histone H3

Beta actin
Beta actin

Figure 17. miR-22 induces DNA damage and accumulates cells in G2/M. A) HCT116 cells were transfected with the indicated miRNAs $(10 \mathrm{nM})$ and incubated for $48 \mathrm{hrs}$. Cells were then subjected to immunoblot. The blots were stained with $\gamma \mathrm{H} 2 \mathrm{AX}$; B \& C) U2OS cells were treated the same as HCT116 cells. Cells were then subjected to flow cytometry to monitor the cell cycle distribution (B) or immunoblot followed by staining with cycline B1 antibody (C); D) U2OS cells were transfected with the indicated miRNAs (10 $\mathrm{nM})$ and incubated for $48 \mathrm{hrs}$. Then, cells were treated with Nocodazole $(100 \mathrm{ng} / \mathrm{ml})$ for another $18 \mathrm{hrs}$. Cells were then subjected to immunoblot followed by staining with phospho-Histone $\mathrm{H} 3$ antibody. 

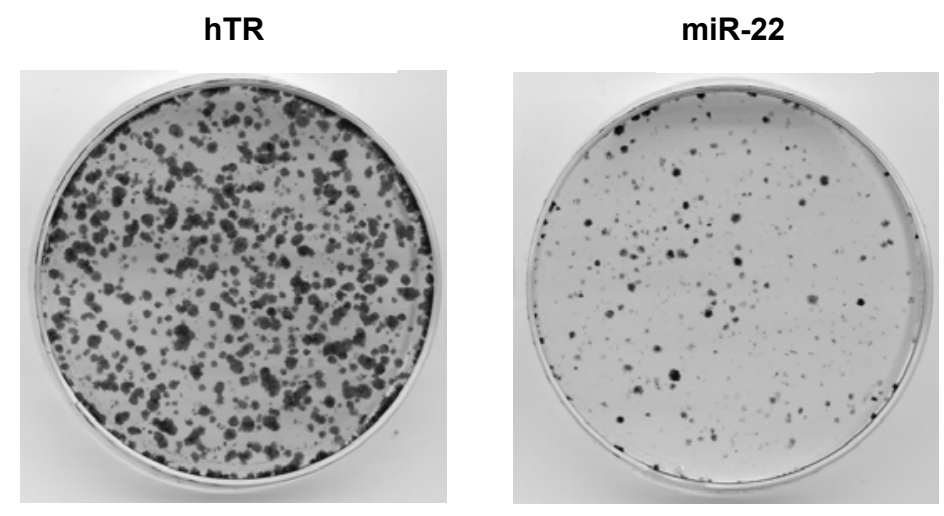

Figure 18. Colony formation assay. U2OS cells were transfected with control miR-vector (hTR) or miR-vec-22. The cultures were maintained for 2 weeks with blasticidin $(5 \mu \mathrm{g} / \mathrm{mL})$ to selecte stable transfectants, and the cells were then fixed, stained with crystal violet, and photographed. 


\subsection{Characterization of p53 modifications induced by miR-101}

\subsection{1 miR-101 increases p53 levels and accumulates modified p53}

One similar immunofluorescence screening performed by my collegue Antje Dickmanns identified miR-101, which enhanced the accumulation of $\gamma \mathrm{H} 2 \mathrm{AX}$, a hallmark of DNA damage, in transfected U2OS cells. Since p53 is a key mediator of the DNA damage response, it is intriguing to examine if miR-101 has any effects on p53. Interestingly, miR-101 induced p53 levels (Fig. 19A), and its target gene products p21 and Mdm2 (Fig. 19B). Furthermore, several upper bands on top of the basic isoform of p53 were detected by a p53 antibody in miR-101 transfected cells (Fig. 19A). This phenomenon was also observed in MCF-7 cells (Fig. 19C). CPT (a genotoxic agent) treatment on top of miR-101 transfection abolished modified p53 (Fig. 19A). This pattern strongly resembles mono-ubiquitinated p53 (Grossman, Deato et al. 2003; Li, Brooks et al. 2003). The C-terminal domain of p53 contains six Lysines which can be mono-ubiquitinated alone or in combination, resulting in several possible increases of total molecular weight. Poly-ubiquitinated p53 has rapid turnover, and it shows a much higher molecular weight on immunoblots. Other ubiquitin-like modifiers include Sumo and Nedd8. Lys386 is capable of undergoing sumoylation, but other Lysines are not. Thus, sumoylated p53 should have only one upper band on immunoblots. However, Nedd8 conjugation could also explain for the pattern observed upon miR-101 transfection since multiple Lysines can undergo neddylation (Xirodimas, Saville et al. 2004). In summary, miR-101 induces p53 levels and its activity, and possibly accumulates p53 ubiquitination or ubiquitin-like modification.

\subsection{2 p53-modification induced by miR-101 is dependent on Mdm2}

We assumed that p53 is mono-ubiquitinated or neddylated upon miR-101 transfection. Mono-ubiquitination is dependent on $\mathrm{Mdm} 2$, and Nedd8 conjugation can also be mediated by Mdm2 (Xirodimas, Saville et al. 2004). Thus, Mdm2 is assumed to have important roles in miR-101 induced p53 modification. To address this, we transfected U2OS cells with miR-101, followed by treatment with Nutlin-3 (a small molecule which inhibit the interaction of Mdm2 and p53). Although the total levels of p53 were up-regulated by Nutlin-3, modified p53 induced by miR-101 was strongly reduced (Fig. 20A). Similar results were also observed when 
A U205
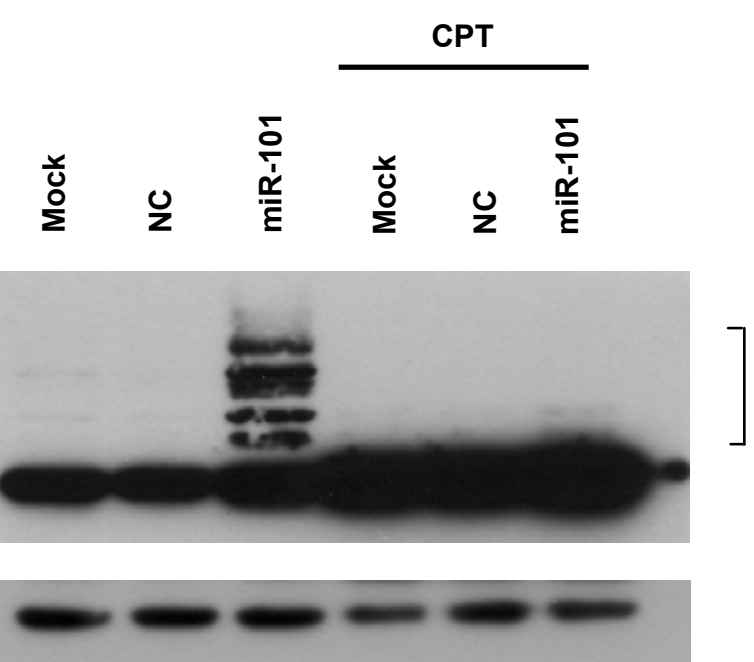

B U20s

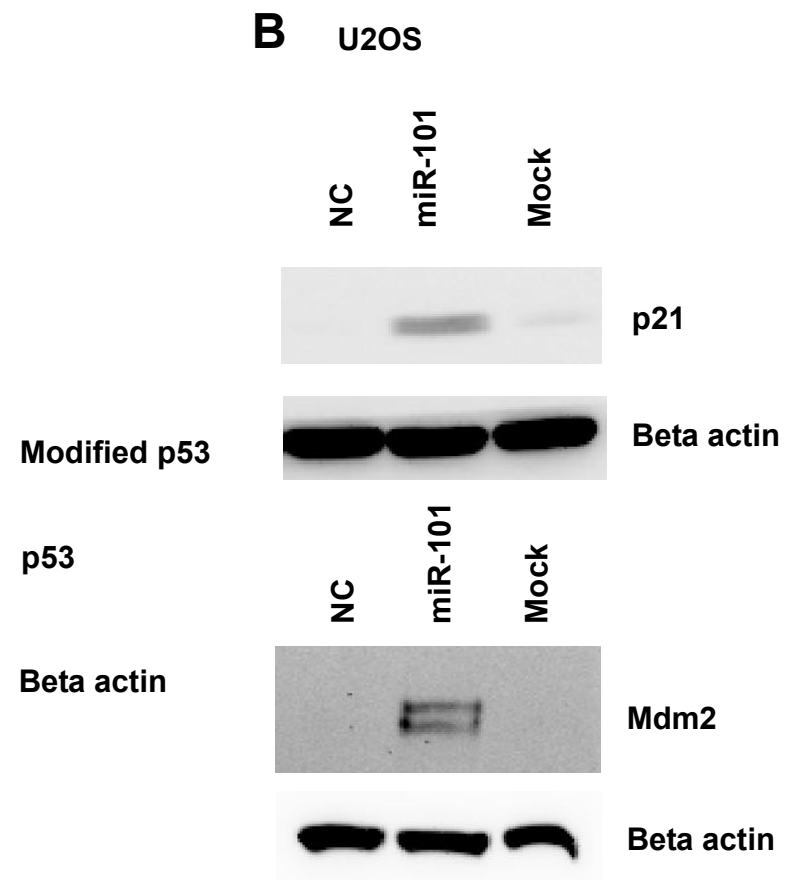

\section{C $\quad$ MCF-7}

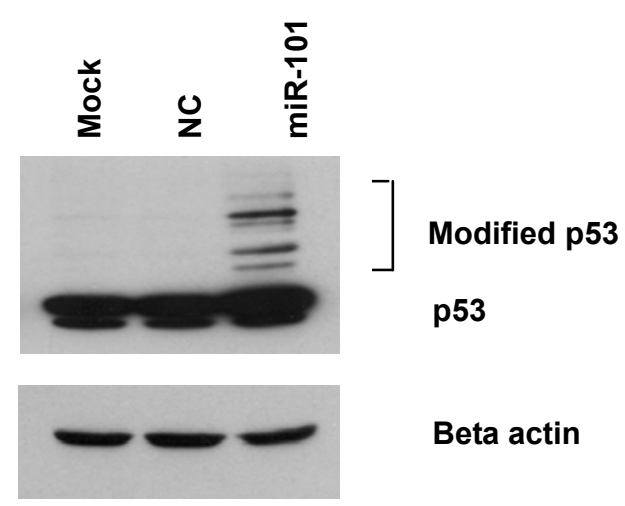

Figure 19. miR-101 induces the levels of p53 and accumulates modified p53. U2OS (A \& B) or MCF-7 (C) cells were transfected with miR-101 (10nM) and incubated for $48 \mathrm{hrs}$. Cells were then harvested and subjected to immunoblot. In the lanes 4-6 of Figure 19A, U2OS cells were transfected with miR-101 $(10 \mathrm{nM}) .36 \mathrm{hrs}$ post-transfection, cells were treated with CPT $(1 \mu \mathrm{M})$ and incubated for another $12 \mathrm{hrs}$. Cells were then harvested and subjected to immunoblot.

the cells were co-transfected with miR-101 and an siRNA against Mdm2, in comparison to a control siRNA (Fig. 20A). Genotoxic stress, which reduces the interaction between Mdm2 and p53, also eliminated modified p53 (Fig. 19A). All together, Mdm2 is a key mediator in miR-101 induced p53 modification. It provided further evidences that this modification should be Mdm2-mediated ubiquitination or neddylation. However, more direct evidences are needed to specify this modification. 
Next, we asked if other p53 modifiers are involved in miR-101 induced p53 modification. p300/CBP was reported to be involved in p53 poly-ubiquitination (Grossman, Deato et al. 2003). USP7 (also called HAUSP) is a deubiquitinase, responsible for p53 and Mdm2 stabilization (Li, Chen et al. 2002; Cummins, Rago et al. 2004; Li, Brooks et al. 2004). USP47 is a homologue of USP7, and is predicted by Targetscan (Lewis, Burge et al. 2005) to be miR-101 target. We assumed that knockdown of each of these p53 modifiers alone might accumulate mono-ubiquitinated p53 and phenocopy miR-101. Thus, we transfected U2OS cells with siRNAs against those p53 modifiers respectively. As shown in Figure 20B, knockdown of any of these p53 modifiers had no obvious effects on p53 modification. However, the efficiency of these siRNAs was not checked. In addition, it is worthwhile examining the effects of combined transfection of siRNAs aginst different p53 modifiers.

\subsection{3 miR-101 suppresses the histone methyltransferase EZH2}

Recently, two groups reported that the histone methyltransferase EZH2 is the target of miR-101 (Varambally, Cao et al. 2008; Friedman, Liang et al. 2009). EZH2 is one member of the Polycomb group (PcG) family, and many PcG proteins are observed to be overexpressed in cancers (Sparmann and van Lohuizen 2006). EZH2 is the catalytic subunit of the polycomb Repressive Complex 2 (PRC2), which mediates gene silencing by trimethylating histone H3 lysine 27 (H3K27me3) (Friedman, Liang et al. 2009). Thus, miR-101 can remodel chromatin via repressing EZH2. Here, we asked if EZH2 is involved in miR-101 induced p53 modification.

First, we confirmed that EZH2 is down-regulated upon miR-101 transfection (Fig. 21A). Then, we transfected U2OS cells with three different siRNAs respectively, all targeting EZH2. All three siRNAs efficiently down-regulated E2H2 (Fig. 21B). The same samples were subjected to immunoblot and stained with p53 antibody. Interestingly, si EZH2 \#2 and \#3 increased p53 levels, whereas si EZH2 \#1 had no effects on p53 (Fig. 21C). Moreover, modified p53, which appeared in the miR-101 transfection experiment, was also observed in si EZH2 \#3 transfected cells (Fig. 21C). Thus, repression of EZH2 by miR-101 might contribute to 553 induction and modification, although we cannot exclude additional off-target effects. 
A miR-101

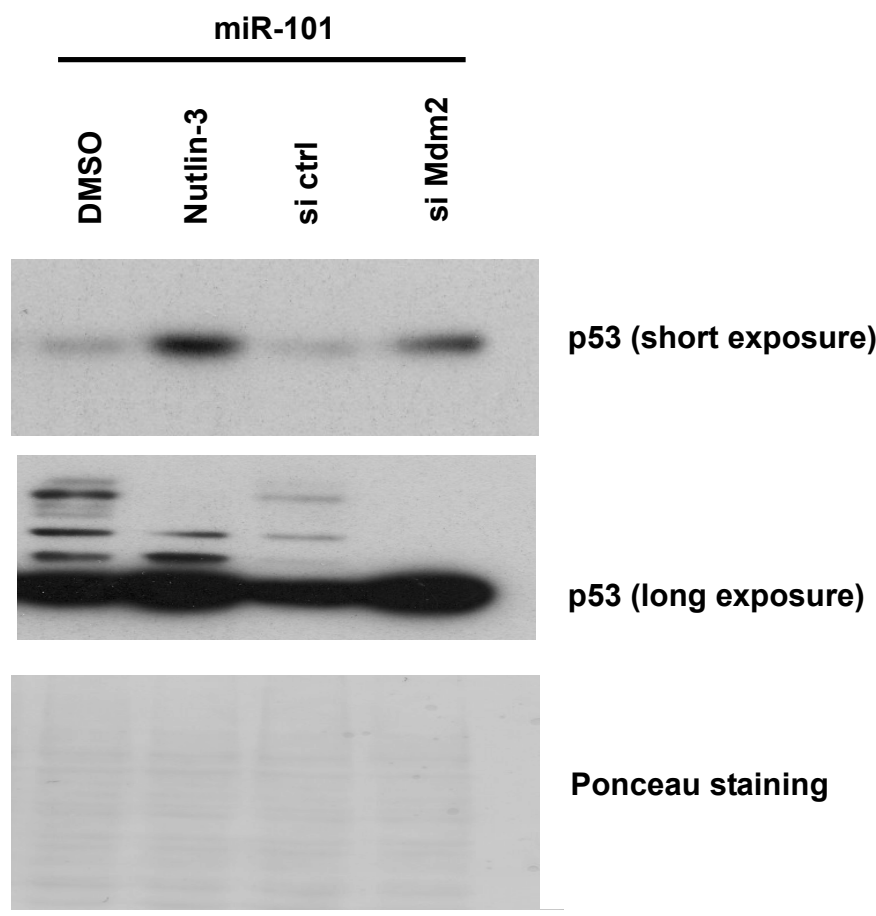

B

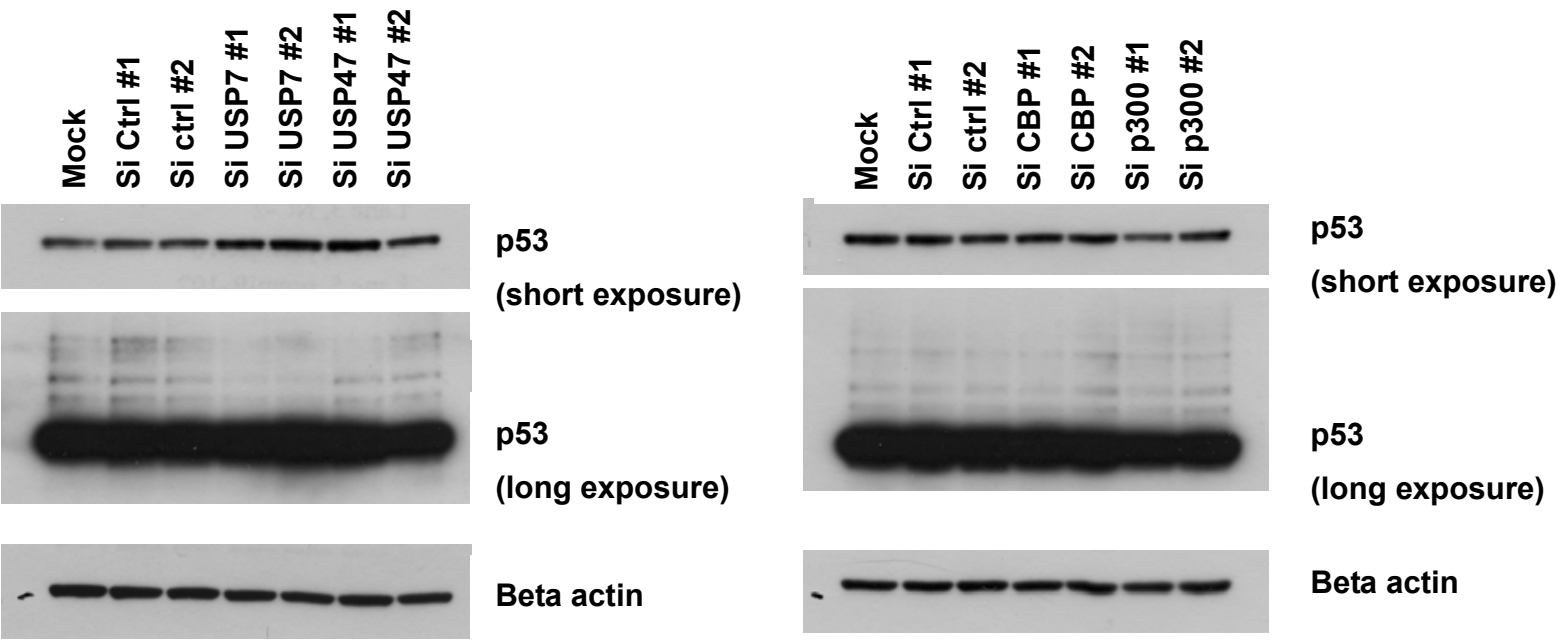

Figure 20. miR-101 induced p53 modification is dependent on Mdm2. A) U2OS cells were transfected with miR-101 alone (Lane $1 \& 2$ ) or along with siRNAs (Lane $3 \& 4$ ). In lane 1 and 2, 24 hrs post-transfection, cells were treated with DMSO or Nutlin-3 $(8 \mu \mathrm{mol} / \mathrm{L})$ for another $27 \mathrm{hrs}$; B) U2OS cells were transfected with siRNAs against p53 modifiers respectively and incubated for $48 \mathrm{hrs}$.

\subsubsection{EZH2 does not detectably associate with p53}

Although the functions of EZH2 in p53 stabilization and modification still need to be verified, we speculated that EZH2 might associate with p53 or Mdm2 and sequester them from each other, by interrupting the interaction between Mdm2 and p53. Furthermore, we assumed that 
EZH2 might methylate Mdm2 or p53 through this association. To test if EZH2 associates with p53, co-immunoprecipitation was performed. As shown in Fig. 22, p53 DO-1 antibody efficiently precipitated p53 proteins, and also an upper band with a size similar to EZH2, but a little bit smaller, was observed. This band could be corresponding to one isoform of EZH2 or a dimer of the IgG heavy chain. To figure it out, an EZH2 antibody was used to precipitate EZH2, and no p53 was co-immunoprecipitated (Fig. 22). Thus, this result suggested that EZH2 does not directly interact with p53. However, the association between EZH2 and Mdm2 still needs to be addressed.

A

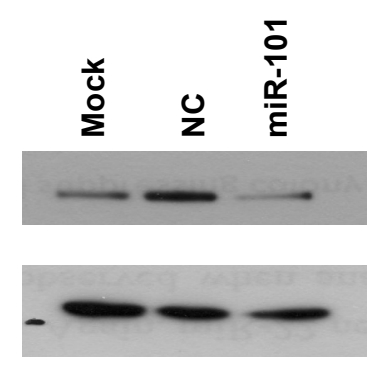

EZH2

Beta actin

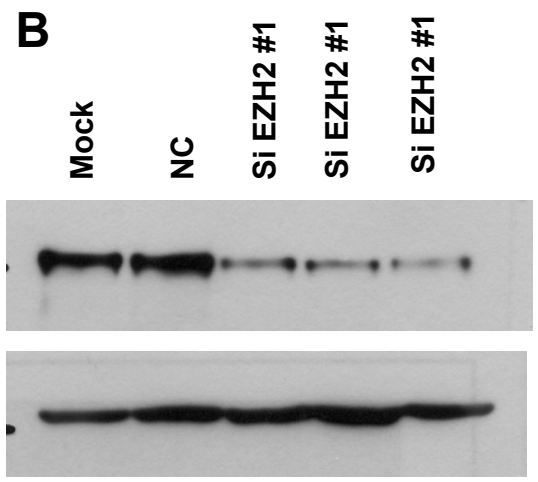

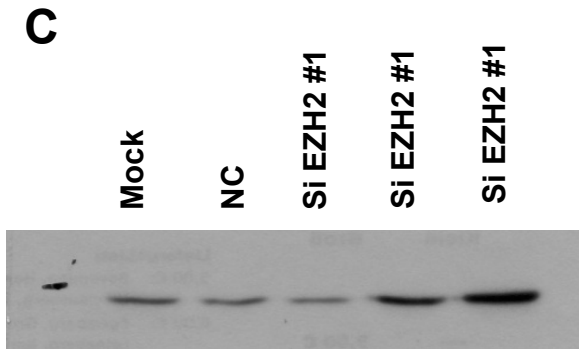

p53

(short exposure)

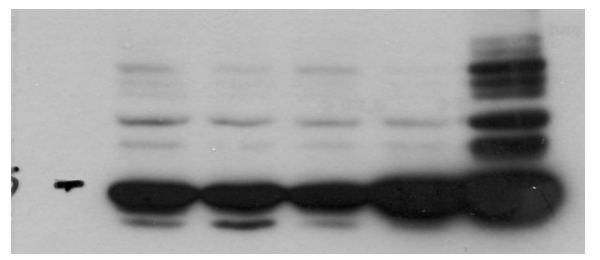

p53

(long exposure)

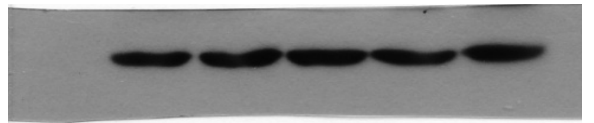

Beta actin

\section{Beta actin}

Figure 21. EZH2 targeted by miR-101 and the effects of EZH2 knockdown on p53. U2OS Cells were transfected with either miR-101 (10 nM) (A) or siRNAs (10 nM) (B \& C) and incubated for $48 \mathrm{hrs}$. 


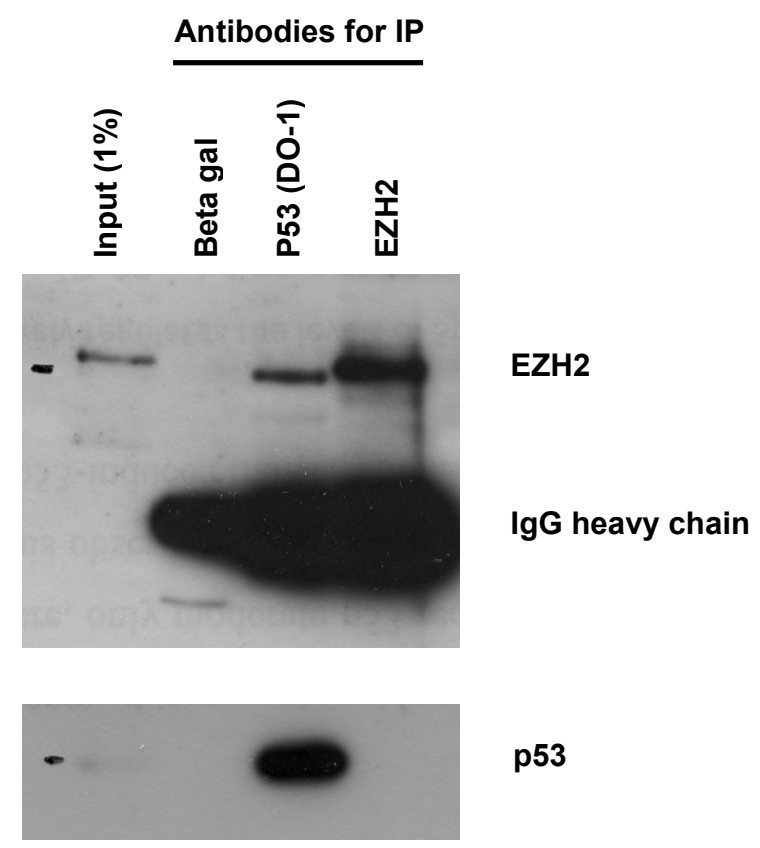

Figure 22. Co-immunoprecipitation assay. U2OS cells were harvested from $10 \mathrm{~cm}$ culture dish and divided into three parts, each subjected to co-immunoprecipitation with Beta gal (lane 2), p53 DO-1 (lane 3) and EZH2 (lane 4), respectively. Immunoblot was first stained with p53 FL393 antibody, and then stained with EZH2 antibody. 


\section{Discussion}

\section{1 miR-192/215 act as p53 effectors and regulators}

\subsection{1 miR-192 is a target gene of p53}

As shown in the studies of miR-34a (Bommer, Gerin et al. 2007; Chang, Wentzel et al. 2007; He, He et al. 2007; Raver-Shapira, Marciano et al. 2007), p53 transactivates miR-34a expression by directly binding to the promoter sequence of miR-34a loci. Thus, p53 regulates not only protein-coding genes, but also non-coding genes. We speculated that miR-192/-215, identified as p53 responsive microRNAs in our study, was also directly regulated by p53. To confirm this, our collegue Braun searched for putative p53 responsive elements in the genome surrounding the two microRNA clusters. A sequence highly similar to the consensus p53 responsive element $2.7 \mathrm{~kb}$ upstream of the miR-194-1/miR-215 cluster was found. Chromatin-immunoprecipitation (ChIP) confirmed the binding of p53 to this site, and this binding was further enhanced when p53 levels were induced by the genotoxic agent camptothecin (Braun, Zhang et al. 2008). Our collegue Sonje Wolff cloned this region into a luciferase reporter plasmid. However, the luciferase activity was not affected by p53 (data not shown). Since the in vitro luciferase assay does not fully resemble the in vivo environment, we cannot exclude this site as a real p53 responsive element. We might have a chance to enhance the responsiveness by cloning several copies of this putative p53 binding sites to the reporter plasmid.

Another issue is that these two clusters are intergenic microRNAs, and we do not know the transcription start sites of these two clusters. If the transcription start site is defined, the promoter region can be located. The difficulty is that primary transcripts of microRNAs are rapidly processed. Based on the assumption that the promoters of miRNAs are conserved, Change et al. generated pairwise alignments between the genomic sequence from human and that from other species to identify the binding sites of Myc (Chang, Yu et al. 2008). It indicated that miRNA promoters may be located a few kilobases to more than $50 \mathrm{~kb}$ upstream of the miRNAs (Chang, Yu et al. 2008).

Song et al. found a putative p53 binding site $3.1 \mathrm{~kb}$ upstream of the miR-194-2/miR-192 cluster (Song, Wang et al. 2008). Chromatin immunoprecipitation and promoter luciferase 
assays further confirmed p53 binding to the promoter of the miR-194-2/miR-192 cluster (Song, Wang et al. 2008). This clearly showed that miR-192 is a direct target gene of p53.

Both miR-34a and miR-192 are p53-responsive miRNAs, but one question is raised: why was miR-192 only identified from our screening, whereas miR-34a was among the lists from all different screening approaches and cell lines? As mentioned in the Introduction, p53 can selectively activate or repress its target genes under various stresses. To fulfill this, p53 needs to recruit co-factors in cooperation with p53 or undergo post-translational modifications. Considering the broad range of biological functions of miR-34a (such as apoptosis, cell cycle arrest and senescence), miR-34a induction is required in diverse responses. Thus, miR-34a induction might only need p53 and general transcription factors. In contrast, miR-192 mainly induces cell cycle arrest. In a subset of stresses, p53 might need co-factors to selectively regulate miR-192 expression. In our screening approach, cells were treated with Nutlin-3, which disrupts the Mdm2-p53 interaction, leading to p53 stabilization without further modifications. Hence, miR-192 induction seems independent of p53 modifications.

\subsubsection{Biological functions of $\mathrm{miR}-192 / 215$}

$\mathrm{p} 53$, as a transcription factor, regulates the expression of many different target genes, leading to different outcomes. Likewise, p53 responsive microRNAs miR-34a and miR-192/215 have distinct functions. The outcomes of miR-34a activation include apoptosis, cell cycle arrest and senescence, which largely depend on the cell type examined. In contrast, miR-192/215 mainly induces G1 or G2/M arrest. miR-192/215 can also induce senescence, but to a much lesser extent relative to miR-34a (Braun, Zhang et al. 2008). Cell detachment was another phenotype observed in HCT116 cells by overexpression of ectopic miR-192/215 (Braun, Zhang et al. 2008). Furthermore, we observed that knockdown of endogenous miR-192 in A549 cells, a lung cancer cell line, rendered cells more sensitive to 5-fluorouracil (5-FU, a genotoxic agent), suggesting miR-192 might provide partial protective functions against DNA damaging agents. In line with this, another group also reported that overexpression of miR-215 in HCT116 cells increased the chemoresistance to methotrexate and Tomudex (two antimetabolite drugs), and knockdown of miR-215 increased chemosensitivity (Song, Wang et al. 2010). 
miRNAs exert their functions mainly through repressing and/or degrading their target mRNAs. To explore the mechanisms how a given miRNA functions, its targets need to be identified. Song et al. reported that dihydrofolate reductase (DHFR) is one target of miR-192 (Song, Wang et al. 2008; Song, Wang et al. 2010). DHFR is a key enzyme responsible for intracellular folate metabolism and a target of methotrexate (Song, Wang et al. 2008). However, it cannot explain how miR-192 halts cell cycle progression. One systematic effort to identify miR-192/215 targets found that miR-192/215 generated a gene expression signature highly enriched for regulators of cell cycle progression (Georges, Biery et al. 2008). Among the 62 genes down-regulated by miR-192, individual knockdown of some of those targets phenocopied miR-192-induced G1 or G2/M arrest. Moreover, these targeted transcripts can regulate cell cycle progression in a cooperative manner.

In addition to regulating cell cycle-related genes as shown by Georges et al., we also found that miR-192/215 induced cell cycle arrest through activating the p53-p21 pathway. p53 and its target gene product p21, other than Puma and Noxa, were induced following miR-192/215 transfection in several cancer cell lines (Braun, Zhang et al. 2008), suggesting that miR-192/215 mostly activates the cell cycle arrest function of p53 other than apoptosis. Furthermore, knockdown of endogenous miR-192 in A549 cells attenuated p53 and p21 activation in response to 5-FU treatment. miR-192/215 induced cell cycle arrest in HCT116 cells, a colon cancer-derived cell line, but not in cells lacking p53 or p21. Thus, the anti-proliferation function of the miR-192/215 is carried out at least in part through the p53-p21 pathway.

How miR-192/215 activates p53 is still an open question. However, the lessons from the studies of miR-34a might give us some hints. Like miR-192/215, miR-34a also provides a positive feedback to the p53 pathway, and some of its functions rely on $\mathrm{p} 53$. Two mechanisms have been proposed to explain how miR-34a activates p53. First, miR-34a causes the down-regulation of the E2F pathway, leading to the up-regulation of the p53-p21 pathway (Tazawa, Tsuchiya et al. 2007). To support this, three groups reported that mouse fibroblasts with triple knockout of E2F1-E2F3 demonstrated inhibited cell proliferation and p53/p21 activation (Wu, Timmers et al. 2001; Sharma, Timmers et al. 2006; Timmers, Sharma et al. 2007). However, the exact mechanism by which E2F1-3 regulates p53 activity is unclear. 
Some claimed that the loss of E2F3 can derepress Arf, leading to activation of p53 and the p53 target gene product p21 (Aslanian, Iaquinta et al. 2004). Others claimed that activation of p53 and p21 by loss of E2F1-3 is due to phosphorylation of p53 at Ser15 (Timmers, Sharma et al. 2007). Second, miR-34a represses SIRT1, leading to p53 activation via increased p53 acetylation. p53 acetylation is an essential event in p53 stabilization and activation. SIRT1, an NAD-dependent deacetylase, has been shown to be involved in regulating p53 functions via deacetylation (Luo, Nikolaev et al. 2001; Vaziri, Dessain et al. 2001; Langley, Pearson et al. 2002; Cheng, Mostoslavsky et al. 2003). Together, miR-34a activates p53 possibly either by recruiting p53 cofactors (e.g. derepressing Arf from E2F pathway) or by post-translational modifications of p53 (e.g. phosphorylation or acetylation).

Likewise, miR-192/215 might affect the p53 pathway through similar mechanisms. Among the confirmed targets of miR-192/215 (Georges, Biery et al. 2008), DTL (one gene encoding denticleless proten homolog) is of particular interest. Two groups reported that DTL associated with the CUL4-DDB1 E3 ubiquitin ligase complex and was involved in the degradation of the replication licensing protein CDT1 in response to DNA damage (Higa, Banks et al. 2006; Sansam, Shepard et al. 2006). DTL is also required for the early G2/M checkpoint (Sansam, Shepard et al. 2006). Further study indicated that DTL can interact with both CUL4-DDB1 and Mdm2 to regulate p53 polyubiquitination, and knockdown of DTL lead to increased levels of p53 and p21 (Banks, Wu et al. 2006). Moreover, it also showed that the CUL4-DDB1 complex was involved in p21 degradation (Nishitani, Shiomi et al. 2008), suggesting that DTL might play more direct roles in regulating p21. Both groups confirmed that DTL is the target of miR-192/215, and knockdown of DTL leads to G2/M arrest (Georges, Biery et al. 2008; Song, Wang et al. 2010). Moreover, knockdown of DTL also leads to p53 activation (Song, Wang et al. 2010). Together, repression of DTL by miR-192/215 contributes to $\mathrm{p} 53$ activation.

Clinical studies further implied the important roles of DTL in tumorigenesis. Among 270 primary hepatocellular carcinomas (HCCs) examined in the study, more than half of the samples showed increased expression of DTL (Pan, Chou et al. 2006). Interestingly, miR-194 (in the same clusters with miR-192/215) was identified as one of the metastasis-related microRNAs in HCCs, and it was down-regulated in metastatic HCC (Budhu, Jia et al. 2008). Thus, the overexpression of DTL in HCCs might be due to loss of miR-192/215. 


\subsection{3 miR-192/215 in cancer}

Considering the biological functions of miR-192/215, they might be potential tumor suppressors. In line with this assumption, we found both miR-192 and miR-215 were down-regulated in colorectal cancer relative to mucosa. Two more groups also compared miRNA profiles of colon tumor and adjacent nontumorous tissues and found both miR-192 and miR-215 with reduced expression in tumors (Schetter, Leung et al. 2008; Earle, Luthra et al. 2010). We found that re-introduction of miR-192/215 inhibited cell proliferation by triggering cell cycle arrest in HCT116 cells, a colon cancer-derived cell line. Furthermore, miR-192/215 is also found to be highly expressed in normal liver and kidney. It is intriguing to examine if there is dysregulation of miR-192/215 in such tumors.

Moreover, miR-192 is located in the chromosome 11q13, which was observed to be deleted in several tumor types, including follicular thyroid tumors (Matsuo, Tang et al. 1991; Zedenius, Wallin et al. 1995; Segev, Saji et al. 1998; Ward, Brenta et al. 1998). MEN1, the gene for multiple endocrine neoplasia type 1 , located in this region, was previously believed to be involved. When Nord et al. investigated the follicular thyroid tumors with 11q13 LOH (loss of heterozygosity), they found a single minimal region of overlapping deletions mapped to the $200 \mathrm{~kb}$ interval between D11S4946 and D11S4939 (Nord, Larsson et al. 1999). They also found that the follicular thyroid tumors with 11q13 LOH had no somatic mutations in MEN1 (Nord, Larsson et al. 1999). This suggested a tumor suppressor gene other than MEN1 in this region plays a role in tumorigenesis of follicular thyroid tumors. Since miR-192 is exactly located in this minimal region, it is worthwhile examining if miR-192 is involved. 


\section{$6.2 \mathrm{miR}-22$, a negative regulator of p53}

\subsection{1 p53 regulation by miR-22}

By using immunofluorescence-based screening in this study, we identified miR-22 as a negative regulator of $\mathrm{p} 53$. We found that miR-22 suppressed p53 levels and activities mainly through two mechanisms: one is to reduce post-translational modifications of p53 (e.g. phosphorylation and acetylation) and the other is to affect the recruitment of the p53 cofactor Sp1.

In response to DNA damage, p53 is firstly modified by phosphorylation at Serine residues located at its N-terminal transactivation domain. Then recruitment of $\mathrm{p} 300 / \mathrm{CBP}$ can facilitate p53 acetylation at its C-terminal Lysine sites. Both modifications can stabilize and activate p53 through disrupting the Mdm2-p53 interaction. As shown in our study, DNA damage-induced p53 phosphorylation at Ser15 and Ser20 and acetylation at Lys382 were significantly impaired by miR-22. Several protein kinases have previously been characterized to be involved in phosphorylating p53 in the DNA damage response. Thus, we proposed that upstream kinases (ATM/ATR, Chk1, Chk2, p38 and others) might be affected by miR-22, leading to impaired p53 phosphorylation. Indeed, both active and global levels of Chk2 were down-regulated by miR-22. Since Chk2 is the downstream target of ATM, ATM might also be involved. But one observation in our study does not favor this idea: miR-22 can induce DNA damage, which should activate ATM. One alternative explanation could be that miR-22 directly targets Chk2 or indirectly affects its transcription because the global levels of Chk2 were also reduced in miR-22-transfected cells. In addition to affect post-translational modifications of p53, miR-22 also impairs p53 activity by down-regulating the p53 co-factor Sp1. It has been shown that Sp1 bound to the $p 21$ promoter leads to activation of p21 gene transcription (Koutsodontis, Tentes et al. 2001).

We conclude that, in addition to the classical p53 modifier (e.g. Mdm2/MdmX, ARF), miRNAs also play important roles in p53 regulation. 


\subsubsection{Cellular functions of miR-22}

Based on the observation that miR-22 suppresses $\mathrm{p} 53$, we asked whether miR-22 is involved in cell proliferation and survival. Surprisingly, miR-22 suppressed clonogenic survival in U2OS cells. Whether p53 is involved in miR-22 suppressed clonogenic survival is one issue to be addressed. One explanation could be that loss of p53 causes replicative stress, leading to the accumulation of DNA damage and subsequent cell death. Indeed, miR-22 induced pronounced DNA damage in HCT116 cells, but it seemed to be at least in part independent on p53. In contrast to promoting G1/S transition due to loss of $\mathrm{p} 53$ as expected, miR-22 accumulated cells in G2/M. Thus, miR-22 suppressed clonogenic survival might be independent of p53. The alternative mechanisms need to be characterized.

We also observed that miR-22 induced senescence-like morphology in HCT116 cells, and triggered $\mathrm{G} 2 / \mathrm{M}$ arrest. p21, a target gene product of $\mathrm{p} 53$, is a key mediator in regulating replicative senescence and cell cycle arrest. However, in our case p21 was repressed by miR-22-mediated suppression of $\mathrm{p} 53$. Hence, there should be some other cell cycle regulators involved. One study indicated that miR-22 repressed the c-Myc binding partner Max, leading to derepressing GADD45 (Ting, Medina et al. 2010). Interestingly, GADD45 is also the target gene product of p53, which is capable of inducing G2/M arrest (Zhan, Antinore et al. 1999; Jin, Tong et al. 2002). GADD45 was also reported to be involved in senescence (Jackson and Pereira-Smith 2006). Together, derepressing of GADD45 from c-Myc by miR-22 might explain miR-22-induced senescence-like morphology and G2/M arrest.

\subsection{3 miR-22 in cancer}

At first glance, miR-22 seems to be an oncogenic miRNA. It not only negatively regulates p53 observed from our study, but also directly targets another tumor suppressor PTEN (Phosphatase and tension homologue deleted on chromosome 10) (Liu, Jiang et al. 2010; Poliseno, Salmena et al. 2010). Poliseno et al. showed that miR-22 was aberrantly overexpressed in human prostate cancer and potentiated cellular transformation both in vitro and in vivo (Poliseno, Salmena et al. 2010). Liu et al. transformed human bronchial epithelial cells with anti anti-benzo[a]pyrene-7,8-diol-9,10-epoxide and found that miR-22 was highly 
expressed in transformed cells (Liu, Jiang et al. 2010). Moreover, they found that knockdown of miR-22 in those transformed cells promoted cell apoptosis, decreased colony formation and reduced the motility of malignant cells (Liu, Jiang et al. 2010). However, both studies did not show if the oncogenic activity of miR-22 is related to the suppression of PTEN or p53.

In contrast, our study clearly showed that miR-22 suppressed clonogenic survival in U2OS cells. In line with our observation, Ting et al. also found that miR-22 inhibited cell proliferation in several cancer cell lines (Ting, Medina et al. 2010). Both groups observed that miR-22 repressed the growth of human estrogen receptor $\alpha(\mathrm{ER} \alpha)$-positive breast cancer cells via directly targeting ER $\alpha$ (Pandey and Picard 2009; Xiong, Yu et al. 2010). Kawahigashi et al. reported that miR-22 was down-regulated in intrahepatic cholangiocarcinoma (ICC, the second most common liver malignancy) cell lines relative to normal intrahepatic biliary epithelial cell line (Kawahigashi, Mishima et al. 2009). Furthermore, Sun et al. found that miR-22 was up-regulated in human pancreatic cancer cells treated with curcumin (a plant-derived anticancer agent) (Sun, Estrov et al. 2008), might explain the mechanism underlying the anticancer activity of curcumin. Moreover, miR-22 is mapped to an exon of C17orf97 gene, located at a fragile cancer-related genomic region $17 \mathrm{p} 13.3$ described in hepatocellular carcinoma (Calin, Sevignani et al. 2004; Xiong, Yu et al. 2010).

\subsubsection{Does c-Myc increase p53 levels by repressing miR-22?}

As a transcription factor, c-Myc can either activate or repress its target genes, resulting in diverse biological outcomes. It has important roles in the control of cell proliferation and transformation, both contributing to its oncogenic activities. Indeed, dysregulation of c-Myc has been observed in many types of human cancer (Oster, Ho et al. 2002). Surprisingly, c-Myc can also induce apoptosis under some conditions (Hoffman and Liebermann 2008). A major mechanism mediating this response is the induction of the tumor suppressor ARF by oncogenic signals, resulting in apoptosis via the ARF-Mdm2-p53 axis (Li and Hann 2009). However, the exact mechanism of how ARF is activated by c-Myc is unclear.

In many tumor cells, ARF is strongly repressed by irreversible epigenetic silencing or eliminated through genetic deletions. But Myc-induced p53 activation is still observed in most 
systems, suggesting that there are alternative pathways independent of ARF. Indeed, one group reported that c-Myc can induce p53 through the ATM-DNA repair pathway rather than ARF (Lindstrom and Wiman 2003). Interestingly, Chang et al. showed that c-Myc represses a broad set of miRNAs, which contributes to tumorigenesis (Chang, Yu et al. 2008). miR-22 is among those repressed miRNAs. Two more studies also suggested that miR-22 might have a regulatory feedback loop to c-Myc (Pandey and Picard 2009; Ting, Medina et al. 2010). Together with our observation that miR-22 negatively regulates $\mathrm{p} 53$, miR-22 provides an attractive link between Myc and p53. We propose that overexpression of Myc inhibits miR-22, resulting in the derepression of p53 (Fig. 18). In line with this, one recent study revealed the roles of miRNAs in the interplay between p53 and c-Myc. miR-145, a newly identified p53 responsive miRNA, directly targets c-Myc, contributing to p53-mediated repression of c-Myc (Sachdeva, Zhu et al. 2009).

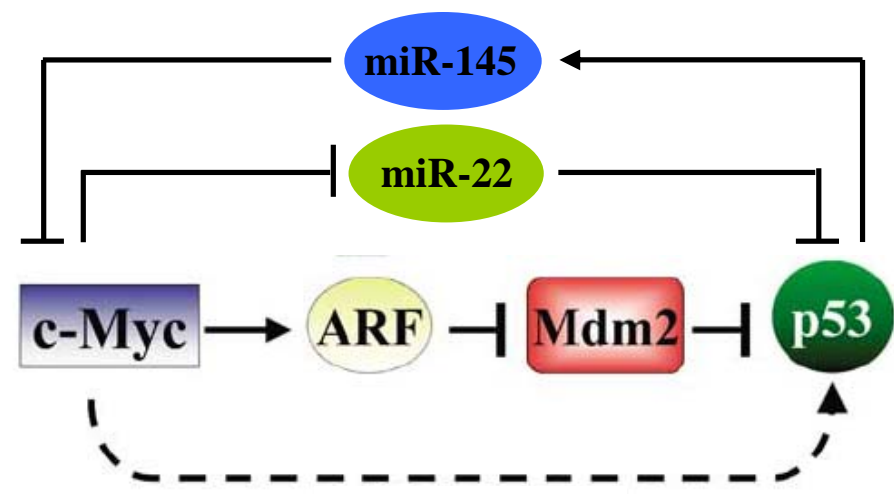

ATM

Figure 23. Interplay between p53 and Myc. In response to Myc, ARF is activated. ARF can either prevent Mdm2 binding to $\mathrm{p} 53$ or inhibit Mdm2 E3 ubiquitin ligase activity, leading to p53 activation. Myc may also activate p53 through ATM-DNA repair pathway. In turn, p53 can repress Myc through miR-145-mediated suppression. Here we propose another link between p53 and Myc: Myc suppresses miR-22, leading to derepressing of p53. Adapted from Nilsson \& Cleveland, 2003. 


\subsection{Post-translational modification of p53 by miR-101 and its biological}

\section{functions}

Post-translational modifications of p53 are key mechanisms in regulating p53 stabilization and activation. In this study, we identified miR-101 as a new p53 modifier. Upon miR-101 transfection in U2OS cells, p53 levels and activities were significantly increased. More surprisingly, accumulation of modified p53 was also observed. This pattern is reminiscent of mono-ubiquitinated p53. Further studies implied that this modification induced by miR-101 was dependent on Mdm2. DNA damage induced by camptothecin (CPT) abolished this modification by miR-101. All these evidences support that miR-101 induces mono-ubiquitinated p53, although more direct evidences are needed. Normally, p53 mono-ubiquitination is an initial event followed by poly-ubiquitination and degradation. How p53 is still stabilized and activated in the form of mono-ubiquitination and in the presence of high levels of Mdm2 upon miR-101 transfection is an intriguing question. It is worthwhile testing if the association of proteasome and Mdm2 or p53 is interfered by miR-101 or not.

Mono-ubiquitinated p53 can be translocated to the mitochondria and play important roles in the intrinsic proapoptotic pathway (Marchenko, Wolff et al. 2007). Thus, miR-101 might function as cell killer by triggering apoptosis. In order to confirm this, we firstly need to analyze the intracellular localization of p53 upon miR-101 transfection. In line with the suggested role of miR-101 in the intrinsic proapoptotic pathway, one group reported that miR-101 may exert its proapoptotic function via targeting Mcl-1 (Su, Yang et al. 2009), an anti-apoptotic member of Bcl-2 family, which prevents the homo-dimerization of Bax.

The mechanism by which miR-101 induces modified p53 is still unclear. It seems to be dependent on Mdm2, so all the factors that can modulate the Mdm2-p53 interaction are within our considerations. We tested some p53 modifiers (such as p300, CBP, HAUSP and its homologue USP47), but they did not seem to be required. We then tested the histone methytransferase EZH2, a well characterized target of miR-101 (Varambally, Cao et al. 2008; Friedman, Liang et al. 2009), although little is known about the roles of EZH2 in the p53 pathway. The result was puzzling since three siRNAs to EZH2 yielded different phenotypes regarding $\mathrm{p} 53$. One siRNA to EZH2 exactly phenocopied the miR-101-induced p53 
modifications, although we could not exclude off-target effects. More siRNAs to EZH2 are definitely needed to verify this effect. And siRNA rescue assays to co-overexpress EZH2 (three to five silent mutations are introduced to the siRNA targeting sequence) with siRNA can be used to test if the phenotype observed by this siRNA is due to off-target effects or not. If knockdown of EZH2 is confirmed to be capable of phenocoping miR-101-induced p53 modification, it would be a fascinating topic for further pursuing. We propose that EZH2 not only methylates Histone proteins but also methylates p53 or Mdm2, and it might even affect the Mdm2-p53 interaction.

What are the functions of miR-101 in cancer? In contrast to miR-22, more consistent data support the idea that miR-101 functions as tumor suppressor. miR-101 has been reported to be down-regulated in cancers of prostate (Varambally, Cao et al. 2008), bladder (Friedman, Liang et al. 2009), and liver ( $\mathrm{Li}, \mathrm{Fu}$ et al. 2009; Su, Yang et al. 2009). This might be due to genomic loss of one or both miR-101 loci (Varambally, Cao et al. 2008). Overexpression of miR-101 can inhibit cell proliferation, invasion, migration and tumor growth (Varambally, Cao et al. 2008; Friedman, Liang et al. 2009; Li, Fu et al. 2009; Su, Yang et al. 2009). miR-101 could also sensitize hepatoma cell lines to both serum starvation- and chemotherapeutic drug-induced apoptosis (Su, Yang et al. 2009). Three miR-101 targets (EZH2, Mcl-1 and FOS) have been identified (Varambally, Cao et al. 2008; Friedman, Liang et al. 2009; Li, Fu et al. 2009; Su, Yang et al. 2009). These three targets can at least partially explain the tumor suppressive activities of miR-101. However, the role of p53 in miR-101-induced tumor suppression has never been reported.

All together, miR-101 can activate p53 and also accumulate modified p53, which might in turn contribute to miR-101-mediated tumor suppression. 


\section{References}

Abbas, T. and A. Dutta (2009). "p21 in cancer: intricate networks and multiple activities." Nat Rev Cancer 9(6): 400-14.

Appella, E. and C. W. Anderson (2001). "Post-translational modifications and activation of p53 by genotoxic stresses." Eur J Biochem 268(10): 2764-72.

Aslanian, A., P. J. laquinta, et al. (2004). "Repression of the Arf tumor suppressor by E2F3 is required for normal cell cycle kinetics." Genes Dev 18(12): 1413-22.

Azuma-Mukai, A., H. Oguri, et al. (2008). "Characterization of endogenous human Argonautes and their miRNA partners in RNA silencing." Proc Natl Acad Sci U S A 105(23): 7964-9.

Banin, S., L. Moyal, et al. (1998). "Enhanced phosphorylation of p53 by ATM in response to DNA damage." Science 281(5383): 1674-7.

Banks, D., M. Wu, et al. (2006). "L2DTL/CDT2 and PCNA interact with p53 and regulate p53 polyubiquitination and protein stability through MDM2 and CUL4A/DDB1 complexes." Cell Cycle 5(15): 1719-29.

Barad, O., E. Meiri, et al. (2004). "MicroRNA expression detected by oligonucleotide microarrays: system establishment and expression profiling in human tissues." Genome Res 14(12): 2486-94.

Barboza, J. A., G. Liu, et al. (2006). "p21 delays tumor onset by preservation of chromosomal stability." Proc Natl Acad Sci U S A 103(52): 19842-7.

Bargonetti, J., J. J. Manfredi, et al. (1993). "A proteolytic fragment from the central region of p53 has marked sequence-specific DNA-binding activity when generated from wild-type but not from oncogenic mutant p53 protein." Genes Dev 7(12B): 2565-74.

Basu, U., K. Si, et al. (2001). "The Saccharomyces cerevisiae TIF6 gene encoding translation initiation factor 6 is required for $60 \mathrm{~S}$ ribosomal subunit biogenesis." Mol Cell Biol 21(5): 1453-62.

Behm-Ansmant, I., J. Rehwinkel, et al. (2006). "mRNA degradation by miRNAs and GW182 requires both CCR4:NOT deadenylase and DCP1:DCP2 decapping complexes." Genes Dev 20(14): 1885-98.

Bergamaschi, D., Y. Samuels, et al. (2003). "iASPP oncoprotein is a key inhibitor of p53 conserved from worm to human." Nat Genet 33(2): 162-7.

Bernstein, E., A. A. Caudy, et al. (2001). "Role for a bidentate ribonuclease in the initiation step of RNA interference." Nature 409(6818): 363-6.

Bohnsack, M. T., K. Regener, et al. (2002). "Exp5 exports eEF1A via tRNA from nuclei and synergizes with other transport pathways to confine translation to the cytoplasm." EMBO J 21(22): 6205-15.

Bommer, G. T., I. Gerin, et al. (2007). "p53-mediated activation of miRNA34 candidate tumor-suppressor genes." Curr Biol 17(15): 1298-307.

Borchert, G. M., W. Lanier, et al. (2006). "RNA polymerase III transcribes human microRNAs." Nat Struct Mol Biol 13(12): 1097-101.

Braastad, C. D., Z. Han, et al. (2003). "Constitutive DNase I hypersensitivity of p53-regulated promoters." J Biol Chem 278(10): 8261-8. 
Braun, C. J., X. Zhang, et al. (2008). "p53-Responsive micrornas 192 and 215 are capable of inducing cell cycle arrest." Cancer Res 68(24): 10094-104.

Briggs, M. R., J. T. Kadonaga, et al. (1986). "Purification and biochemical characterization of the promoter-specific transcription factor, Sp1." Science 234(4772): 47-52.

Brown, J. P., W. Wei, et al. (1997). "Bypass of senescence after disruption of p21CIP1/WAF1 gene in normal diploid human fibroblasts." Science 277(5327): 831-4.

Brugarolas, J., C. Chandrasekaran, et al. (1995). "Radiation-induced cell cycle arrest compromised by p21 deficiency." Nature 377(6549): 552-7.

Brummelkamp, T. R., R. Bernards, et al. (2002). "A system for stable expression of short interfering RNAs in mammalian cells." Science 296(5567): 550-3.

Budhu, A., H. L. Jia, et al. (2008). "Identification of metastasis-related microRNAs in hepatocellular carcinoma." Hepatology 47(3): 897-907.

Bullrich, F., H. Fujii, et al. (2001). "Characterization of the $13 q 14$ tumor suppressor locus in CLL: identification of ALT1, an alternative splice variant of the LEU2 gene." Cancer Res 61(18): 6640-8.

Cai, X., C. H. Hagedorn, et al. (2004). "Human microRNAs are processed from capped, polyadenylated transcripts that can also function as mRNAs." RNA 10(12): 1957-66.

Calado, A., N. Treichel, et al. (2002). "Exportin-5-mediated nuclear export of eukaryotic elongation factor $1 \mathrm{~A}$ and tRNA." EMBO J 21(22): 6216-24.

Calin, G. A., C. D. Dumitru, et al. (2002). "Frequent deletions and down-regulation of micro- RNA genes miR15 and miR16 at 13q14 in chronic lymphocytic leukemia." Proc Natl Acad Sci U S A 99(24): 15524-9.

Calin, G. A., M. Ferracin, et al. (2005). "A MicroRNA signature associated with prognosis and progression in chronic lymphocytic leukemia." $\mathrm{N}$ Engl J Med 353(17): 1793-801.

Calin, G. A., C. Sevignani, et al. (2004). "Human microRNA genes are frequently located at fragile sites and genomic regions involved in cancers." Proc Natl Acad Sci U S A 101(9): 2999-3004.

Canman, C. E., D. S. Lim, et al. (1998). "Activation of the ATM kinase by ionizing radiation and phosphorylation of p53." Science 281(5383): 1677-9.

Chang, B. D., K. Watanabe, et al. (2000). "Effects of p21Waf1/Cip1/Sdi1 on cellular gene expression: implications for carcinogenesis, senescence, and age-related diseases." Proc Natl Acad Sci U S A 97(8): 4291-6.

Chang, T. C., E. A. Wentzel, et al. (2007). "Transactivation of miR-34a by p53 broadly influences gene expression and promotes apoptosis." Mol Cell 26(5): 745-52.

Chang, T. C., D. Yu, et al. (2008). "Widespread microRNA repression by Myc contributes to tumorigenesis." Nat Genet 40(1): 43-50.

Chendrimada, T. P., K. J. Finn, et al. (2007). "MicroRNA silencing through RISC recruitment of elF6." Nature 447(7146): 823-8.

Chendrimada, T. P., R. I. Gregory, et al. (2005). "TRBP recruits the Dicer complex to Ago2 for microRNA processing and gene silencing." Nature 436(7051): 740-4. 
Cheng, H. L., R. Mostoslavsky, et al. (2003). "Developmental defects and p53 hyperacetylation in Sir2 homolog (SIRT1)-deficient mice." Proc Natl Acad Sci U S A 100(19): 10794-9.

Chipuk, J. E., L. Bouchier-Hayes, et al. (2005). "PUMA couples the nuclear and cytoplasmic proapoptotic function of p53." Science 309(5741): 1732-5.

Contente, A., A. Dittmer, et al. (2002). "A polymorphic microsatellite that mediates induction of PIG3 by p53." Nat Genet 30(3): 315-20.

Corney, D. C., A. Flesken-Nikitin, et al. (2007). "MicroRNA-34b and MicroRNA-34c are targets of p53 and cooperate in control of cell proliferation and adhesion-independent growth." Cancer Res 67(18): 8433-8.

Craig, A. L., L. Burch, et al. (1999). "Novel phosphorylation sites of human tumour suppressor protein p53 at Ser20 and Thr18 that disrupt the binding of mdm2 (mouse double minute 2) protein are modified in human cancers." Biochem J 342 ( Pt 1): 133-41.

Craig, A. L. and T. R. Hupp (2004). "The regulation of CHK2 in human cancer." Oncogene 23(52): 8411-8.

Cummins, J. M., C. Rago, et al. (2004). "Tumour suppression: disruption of HAUSP gene stabilizes p53." Nature 428(6982): 1 p following 486.

Das, S., L. Raj, et al. (2007). "Hzf Determines cell survival upon genotoxic stress by modulating p53 transactivation." Cell 130(4): 624-37.

Davis, B. N., A. C. Hilyard, et al. (2008). "SMAD proteins control DROSHA-mediated microRNA maturation." Nature 454(7200): 56-61.

Delavaine, L. and N. B. La Thangue (1999). "Control of E2F activity by p21Waf1/Cip1." Oncogene 18(39): 5381-92.

Deng, C., P. Zhang, et al. (1995). "Mice lacking p21CIP1/WAF1 undergo normal development, but are defective in G1 checkpoint control." Cell 82(4): 675-84.

Deng, Y., S. S. Chan, et al. (2008). "Telomere dysfunction and tumour suppression: the senescence connection." Nat Rev Cancer 8(6): 450-8.

Denli, A. M., B. B. Tops, et al. (2004). "Processing of primary microRNAs by the Microprocessor complex." Nature 432(7014): 231-5.

Diederichs, S. and D. A. Haber (2007). "Dual role for argonautes in microRNA processing and posttranscriptional regulation of microRNA expression." Cell 131(6): 1097-108.

Dohner, H., S. Stilgenbauer, et al. (2000). "Genomic aberrations and survival in chronic lymphocytic leukemia." N Engl J Med 343(26): 1910-6.

Donehower, L. A., M. Harvey, et al. (1992). "Mice deficient for p53 are developmentally normal but susceptible to spontaneous tumours." Nature 356(6366): 215-21.

Earle, J. S., R. Luthra, et al. (2010). "Association of MicroRNA Expression with Microsatellite Instability Status in Colorectal Adenocarcinoma." J Mol Diagn.

el-Deiry, W. S. (1998). "Regulation of p53 downstream genes." Semin Cancer Biol 8(5): 345-57.

Esquela-Kerscher, A. and F. J. Slack (2006). "Oncomirs - microRNAs with a role in cancer." Nat Rev Cancer 6(4): 259-69. 
Eulalio, A., E. Huntzinger, et al. (2008). "Getting to the root of miRNA-mediated gene silencing." Cell 132(1): 9-14.

Eulalio, A., J. Rehwinkel, et al. (2007). "Target-specific requirements for enhancers of decapping in miRNA-mediated gene silencing." Genes Dev 21(20): 2558-70.

Filipowicz, W., S. N. Bhattacharyya, et al. (2008). "Mechanisms of post-transcriptional regulation by microRNAs: are the answers in sight?" Nat Rev Genet 9(2): 102-14.

Forman, J. J., A. Legesse-Miller, et al. (2008). "A search for conserved sequences in coding regions reveals that the let-7 microRNA targets Dicer within its coding sequence." Proc Natl Acad Sci U S A 105(39): 14879-84.

Forstemann, K., M. D. Horwich, et al. (2007). "Drosophila microRNAs are sorted into functionally distinct argonaute complexes after production by dicer-1." Cell 130(2): 287-97.

Friedman, J. M., G. Liang, et al. (2009). "The putative tumor suppressor microRNA-101 modulates the cancer epigenome by repressing the polycomb group protein EZH2." Cancer Res 69(6): 2623-9.

Gaur, A., D. A. Jewell, et al. (2007). "Characterization of microRNA expression levels and their biological correlates in human cancer cell lines." Cancer Res 67(6): 2456-68. Georges, S. A., M. C. Biery, et al. (2008). "Coordinated regulation of cell cycle transcripts by p53-Inducible microRNAs, miR-192 and miR-215." Cancer Res 68(24): 10105-12.

Giraldez, A. J., Y. Mishima, et al. (2006). "Zebrafish MiR-430 promotes deadenylation and clearance of maternal mRNAs." Science 312(5770): 75-9.

Gregory, R. I., K. P. Yan, et al. (2004). "The Microprocessor complex mediates the genesis of microRNAs." Nature 432(7014): 235-40.

Grishok, A., A. E. Pasquinelli, et al. (2001). "Genes and mechanisms related to RNA interference regulate expression of the small temporal RNAs that control C. elegans developmental timing." Cell 106(1): 23-34.

Grossman, S. R., M. E. Deato, et al. (2003). "Polyubiquitination of p53 by a ubiquitin ligase activity of $\mathrm{p} 300 . "$ Science 300(5617): 342-4.

Guang, S., A. F. Bochner, et al. (2008). "An Argonaute transports siRNAs from the cytoplasm to the nucleus." Science 321(5888): 537-41.

Guil, S. and J. F. Caceres (2007). "The multifunctional RNA-binding protein hnRNP A1 is required for processing of miR-18a." Nat Struct Mol Biol 14(7): 591-6.

Han, J., Y. Lee, et al. (2004). "The Drosha-DGCR8 complex in primary microRNA processing." Genes Dev 18(24): 3016-27.

Han, J., Y. Lee, et al. (2006). "Molecular basis for the recognition of primary microRNAs by the Drosha-DGCR8 complex." Cell 125(5): 887-901.

Han, J., J. S. Pedersen, et al. (2009). "Posttranscriptional crossregulation between Drosha and DGCR8." Cell 136(1): 75-84.

Harley, C. B., A. B. Futcher, et al. (1990). "Telomeres shorten during ageing of human fibroblasts." Nature 345(6274): 458-60.

Hayflick, L. and P. S. Moorhead (1961). "The serial cultivation of human diploid cell strains." Exp Cell Res 25: 585-621. 
He, L., X. He, et al. (2007). "A microRNA component of the p53 tumour suppressor network." Nature 447(7148): 1130-4.

Heo, I., C. Joo, et al. (2008). "Lin28 mediates the terminal uridylation of let-7 precursor MicroRNA." Mol Cell 32(2): 276-84.

Higa, L. A., D. Banks, et al. (2006). "L2DTL/CDT2 interacts with the CUL4/DDB1 complex and PCNA and regulates CDT1 proteolysis in response to DNA damage." Cell Cycle 5(15): 1675-80.

Hoffman, B. and D. A. Liebermann (2008). "Apoptotic signaling by c-MYC." Oncogene 27(50): 6462-72.

Honda, R. and H. Yasuda (1999). "Association of p19(ARF) with Mdm2 inhibits ubiquitin ligase activity of Mdm2 for tumor suppressor p53." EMBO J 18(1): 22-7.

Humphreys, D. T., B. J. Westman, et al. (2005). "MicroRNAs control translation initiation by inhibiting eukaryotic initiation factor $4 \mathrm{E} / \mathrm{cap}$ and poly $(\mathrm{A})$ tail function." Proc Natl Acad Sci U S A 102(47): 16961-6.

Hutvagner, G., J. McLachlan, et al. (2001). "A cellular function for the RNA-interference enzyme Dicer in the maturation of the let-7 small temporal RNA." Science 293(5531): 834-8.

Hwang, H. W., E. A. Wentzel, et al. (2007). "A hexanucleotide element directs microRNA nuclear import." Science 315(5808): 97-100.

lacopetta, B. (2003). "TP53 mutation in colorectal cancer." Hum Mutat 21(3): 271-6. Inga, A., F. Storici, et al. (2002). "Differential transactivation by the p53 transcription factor is highly dependent on p53 level and promoter target sequence." Mol Cell Biol 22(24): 8612-25.

lorio, M. V., M. Ferracin, et al. (2005). "MicroRNA gene expression deregulation in human breast cancer." Cancer Res 65(16): 7065-70.

Ito, A., C. H. Lai, et al. (2001). "p300/CBP-mediated p53 acetylation is commonly induced by p53-activating agents and inhibited by MDM2." EMBO J 20(6): 1331-40.

Ivanovska, I., A. S. Ball, et al. (2008). "MicroRNAs in the miR-106b family regulate p21/CDKN1A and promote cell cycle progression." Mol Cell Biol 28(7): 2167-74.

Jackson, J. G. and O. M. Pereira-Smith (2006). "p53 is preferentially recruited to the promoters of growth arrest genes p21 and GADD45 during replicative senescence of normal human fibroblasts." Cancer Res 66(17): 8356-60.

Jin, S., T. Tong, et al. (2002). "GADD45-induced cell cycle G2-M arrest associates with altered subcellular distribution of cyclin B1 and is independent of p38 kinase activity." Oncogene 21(57): 8696-704.

Johnson, S. M., H. Grosshans, et al. (2005). "RAS is regulated by the let-7 microRNA family." Cell 120(5): 635-47.

Jones, S. N., A. E. Roe, et al. (1995). "Rescue of embryonic lethality in Mdm2-deficient mice by absence of p53." Nature 378(6553): 206-8.

Jopling, C. L., M. Yi, et al. (2005). "Modulation of hepatitis C virus RNA abundance by a liver-specific MicroRNA." Science 309(5740): 1577-81.

Kadonaga, J. T., K. R. Carner, et al. (1987). "Isolation of cDNA encoding transcription factor Sp1 and functional analysis of the DNA binding domain." Cell 51(6): 1079-90. 
Kawahigashi, Y., T. Mishima, et al. (2009). "MicroRNA profiling of human intrahepatic cholangiocarcinoma cell lines reveals biliary epithelial cell-specific microRNAs." $\underline{J}$ Nippon Med Sch 76(4): 188-97.

Kawai, H., D. Wiederschain, et al. (2003). "DNA damage-induced MDMX degradation is mediated by MDM2." J Biol Chem 278(46): 45946-53.

Ketting, R. F., S. E. Fischer, et al. (2001). "Dicer functions in RNA interference and in synthesis of small RNA involved in developmental timing in C. elegans." Genes Dev 15(20): 2654-9.

Khanna, K. K., K. E. Keating, et al. (1998). "ATM associates with and phosphorylates p53: mapping the region of interaction." Nat Genet 20(4): 398-400.

Khvorova, A., A. Reynolds, et al. (2003). "Functional siRNAs and miRNAs exhibit strand bias." Cell 115(2): 209-16.

Kim, D. H., P. Saetrom, et al. (2008). "MicroRNA-directed transcriptional gene silencing in mammalian cells." Proc Natl Acad Sci U S A 105(42): 16230-5.

Kim, E. and W. Deppert (2003). "The complex interactions of p53 with target DNA: we learn as we go." Biochem Cell Biol 81(3): 141-50.

Kim, G. P., L. H. Colangelo, et al. (2007). "Prognostic and predictive roles of high-degree microsatellite instability in colon cancer: a National Cancer Institute-National Surgical Adjuvant Breast and Bowel Project Collaborative Study." $\underline{J}$ Clin Oncol 25(7): 767-72.

Kim, V. N. (2005). "MicroRNA biogenesis: coordinated cropping and dicing." Nat Rev Mol Cell Biol 6(5): 376-85.

Kim, Y. K. and V. N. Kim (2007). "Processing of intronic microRNAs." EMBO J 26(3): 775-83.

Kiriakidou, M., G. S. Tan, et al. (2007). "An mRNA m7G cap binding-like motif within human Ago2 represses translation." Cell 129(6): 1141-51.

Kloosterman, W. P. and R. H. Plasterk (2006). "The diverse functions of microRNAs in animal development and disease." Dev Cell 11(4): 441-50.

Knights, C. D., J. Catania, et al. (2006). "Distinct p53 acetylation cassettes differentially influence gene-expression patterns and cell fate." J Cell Biol 173(4): 533-44.

Kortlever, R. M., P. J. Higgins, et al. (2006). "Plasminogen activator inhibitor-1 is a critical downstream target of p53 in the induction of replicative senescence." Nat Cell Biol 8(8): 877-84.

Koutsodontis, G., I. Tentes, et al. (2001). "Sp1 plays a critical role in the transcriptional activation of the human cyclin-dependent kinase inhibitor p21(WAF1/Cip1) gene by the p53 tumor suppressor protein." J Biol Chem 276(31): 29116-25.

Kruse, J. P. and W. Gu (2009). "Modes of p53 regulation." Cell 137(4): 609-22.

Lakin, N. D., B. C. Hann, et al. (1999). "The ataxia-telangiectasia related protein ATR mediates DNA-dependent phosphorylation of p53." Oncogene 18(27): 3989-95.

Lane, D. P. (1992). "Cancer. p53, guardian of the genome." Nature 358(6381): 15-6.

Langley, E., M. Pearson, et al. (2002). "Human SIR2 deacetylates p53 and antagonizes PML/p53-induced cellular senescence." EMBO J 21(10): 2383-96.

Laptenko, O. and C. Prives (2006). "Transcriptional regulation by p53: one protein, many possibilities." Cell Death Differ 13(6): 951-61. 
Lavin, M. F. and N. Gueven (2006). "The complexity of p53 stabilization and activation." Cell Death Differ 13(6): 941-50.

Le, M. T., H. Xie, et al. (2009). "MicroRNA-125b promotes neuronal differentiation in human cells by repressing multiple targets." Mol Cell Biol 29(19): 5290-305.

Leal, J. F., J. Fominaya, et al. (2008). "Cellular senescence bypass screen identifies new putative tumor suppressor genes." Oncogene 27(14): 1961-70.

Lee, Y., C. Ahn, et al. (2003). "The nuclear RNase III Drosha initiates microRNA processing." Nature 425(6956): 415-9.

Lee, Y., I. Hur, et al. (2006). "The role of PACT in the RNA silencing pathway." EMBO J 25(3): 522-32.

Lee, Y., M. Kim, et al. (2004). "MicroRNA genes are transcribed by RNA polymerase II." EMBO J 23(20): 4051-60.

Levine, A. J. (1997). "p53, the cellular gatekeeper for growth and division." Cell 88(3): 323-31.

Lewis, B. P., C. B. Burge, et al. (2005). "Conserved seed pairing, often flanked by adenosines, indicates that thousands of human genes are microRNA targets." Cell 120(1): 15-20.

Li, L. C., S. T. Okino, et al. (2006). "Small dsRNAs induce transcriptional activation in human cells." Proc Natl Acad Sci U S A 103(46): 17337-42.

Li, M., C. L. Brooks, et al. (2004). "A dynamic role of HAUSP in the p53-Mdm2 pathway." Mol Cell 13(6): 879-86.

Li, M., C. L. Brooks, et al. (2003). "Mono- versus polyubiquitination: differential control of p53 fate by Mdm2." Science 302(5652): 1972-5.

$\mathrm{Li}, \mathrm{M}$., D. Chen, et al. (2002). "Deubiquitination of p53 by HAUSP is an important pathway for p53 stabilization." Nature 416(6881): 648-53.

Li, M., J. Luo, et al. (2002). "Acetylation of p53 inhibits its ubiquitination by Mdm2." $\underline{\mathrm{J}}$ Biol Chem 277(52): 50607-11.

$\mathrm{Li}, \mathrm{S}$., H. Fu, et al. (2009). "MicroRNA-101 regulates expression of the v-fos FBJ murine osteosarcoma viral oncogene homolog (FOS) oncogene in human hepatocellular carcinoma." Hepatology 49(4): 1194-202.

Li, Z. and S. R. Hann (2009). "The Myc-nucleophosmin-ARF network: a complex web unveiled." Cell Cycle 8(17): 2703-7.

Linares, L. K., A. Hengstermann, et al. (2003). "HdmX stimulates Hdm2-mediated ubiquitination and degradation of p53." Proc Natl Acad Sci U S A 100(21): 12009-14.

Lindstrom, M. S. and K. G. Wiman (2003). "Myc and E2F1 induce p53 through p14ARF-independent mechanisms in human fibroblasts." Oncogene 22(32): 4993-5005.

Liu, J., M. A. Carmell, et al. (2004). "Argonaute2 is the catalytic engine of mammalian RNAi." Science 305(5689): 1437-41.

Liu, L., Y. Jiang, et al. (2010). "miR-22 functions as a micro-oncogene in transformed human bronchial epithelial cells induced by anti-benzo[a]pyrene-7,8-diol-9,10epoxide." Toxicol In Vitro.

Lu, J., G. Getz, et al. (2005). "MicroRNA expression profiles classify human cancers." Nature 435(7043): 834-8. 
Lund, E., S. Guttinger, et al. (2004). "Nuclear export of microRNA precursors." Science 303(5654): 95-8.

Luo, J., A. Y. Nikolaev, et al. (2001). "Negative control of p53 by Sir2alpha promotes cell survival under stress." Cell 107(2): 137-48.

Marchenko, N. D., S. Wolff, et al. (2007). "Monoubiquitylation promotes mitochondrial p53 translocation." EMBO J 26(4): 923-34.

Marine, J. C. and A. G. Jochemsen (2005). "Mdmx as an essential regulator of p53 activity." Biochem Biophys Res Commun 331(3): 750-60.

Maroney, P. A., Y. Yu, et al. (2006). "Evidence that microRNAs are associated with translating messenger RNAs in human cells." Nat Struct Mol Biol 13(12): 1102-7.

Mathonnet, G., M. R. Fabian, et al. (2007). "MicroRNA inhibition of translation initiation in vitro by targeting the cap-binding complex elF4F." Science 317(5845): 1764-7.

Matsuo, K., S. H. Tang, et al. (1991). "Allelotype of human thyroid tumors: loss of chromosome 11q13 sequences in follicular neoplasms." Mol Endocrinol 5(12): 1873-9. Meister, G., M. Landthaler, et al. (2004). "Sequence-specific inhibition of microRNAand siRNA-induced RNA silencing." RNA 10(3): 544-50.

Meulmeester, E., M. M. Maurice, et al. (2005). "Loss of HAUSP-mediated deubiquitination contributes to DNA damage-induced destabilization of Hdmx and Hdm2." Mol Cell 18(5): 565-76.

Migliorini, D., E. Lazzerini Denchi, et al. (2002). "Mdm4 (Mdmx) regulates p53-induced growth arrest and neuronal cell death during early embryonic mouse development." Mol Cell Biol 22(15): 5527-38.

Minsky, N. and M. Oren (2004). "The RING domain of Mdm2 mediates histone ubiquitylation and transcriptional repression." Mol Cell 16(4): 631-9.

Moll, U. M., S. Wolff, et al. (2005). "Transcription-independent pro-apoptotic functions of p53." Curr Opin Cell Biol 17(6): 631-6.

Momand, J., G. P. Zambetti, et al. (1992). "The mdm-2 oncogene product forms a complex with the p53 protein and inhibits p53-mediated transactivation." Cell 69(7): 1237-45.

Montes de Oca Luna, R., D. S. Wagner, et al. (1995). "Rescue of early embryonic lethality in mdm2-deficient mice by deletion of p53." Nature 378(6553): 203-6.

Naar, A. M., S. Ryu, et al. (1998). "Cofactor requirements for transcriptional activation by Sp1." Cold Spring Harb Symp Quant Biol 63: 189-99.

Newman, M. A., J. M. Thomson, et al. (2008). "Lin-28 interaction with the Let-7 precursor loop mediates regulated microRNA processing." RNA 14(8): 1539-49.

Nishitani, H., Y. Shiomi, et al. (2008). "CDK inhibitor p21 is degraded by a proliferating cell nuclear antigen-coupled Cul4-DDB1Cdt2 pathway during S phase and after UV irradiation." J Biol Chem 283(43): 29045-52.

Noma, K., T. Sugiyama, et al. (2004). "RITS acts in cis to promote RNA interference-mediated transcriptional and post-transcriptional silencing." Nat Genet 36(11): 1174-80.

Nord, B., C. Larsson, et al. (1999). "Sporadic follicular thyroid tumors show loss of a 200-kb region in 11q13 without evidence for mutations in the MEN1 gene." Genes Chromosomes Cancer 26(1): 35-9. 
Nottrott, S., M. J. Simard, et al. (2006). "Human let-7a miRNA blocks protein production on actively translating polyribosomes." Nat Struct Mol Biol 13(12): 1108-14.

Oda, K., H. Arakawa, et al. (2000). "p53AIP1, a potential mediator of p53-dependent apoptosis, and its regulation by Ser-46-phosphorylated p53." Cell 102(6): 849-62.

O'Donnell, K. A., E. A. Wentzel, et al. (2005). "c-Myc-regulated microRNAs modulate E2F1 expression." Nature 435(7043): 839-43.

Okamura, K., J. W. Hagen, et al. (2007). "The mirtron pathway generates microRNA-class regulatory RNAs in Drosophila." Cell 130(1): 89-100.

Oliner, J. D., K. W. Kinzler, et al. (1992). "Amplification of a gene encoding a p53-associated protein in human sarcomas." Nature 358(6381): 80-3.

Oliner, J. D., J. A. Pietenpol, et al. (1993). "Oncoprotein MDM2 conceals the activation domain of tumour suppressor p53." Nature 362(6423): 857-60.

Oster, S. K., C. S. Ho, et al. (2002). "The myc oncogene: MarvelouslY Complex." Adv Cancer Res 84: 81-154.

Ota, A., H. Tagawa, et al. (2004). "Identification and characterization of a novel gene, C13orf25, as a target for 13q31-q32 amplification in malignant lymphoma." Cancer Res 64(9): 3087-95.

Pan, H. W., H. Y. Chou, et al. (2006). "Role of L2DTL, cell cycle-regulated nuclear and

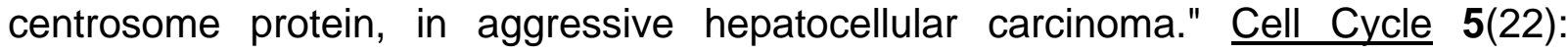
2676-87.

Pan, Y. and J. Chen (2003). "MDM2 promotes ubiquitination and degradation of MDMX." Mol Cell Biol 23(15): 5113-21.

Pandey, D. P. and D. Picard (2009). "miR-22 inhibits estrogen signaling by directly targeting the estrogen receptor alpha mRNA." Mol Cell Biol 29(13): 3783-90.

Parant, J., A. Chavez-Reyes, et al. (2001). "Rescue of embryonic lethality in Mdm4-null mice by loss of Trp53 suggests a nonoverlapping pathway with MDM2 to regulate p53." Nat Genet 29(1): 92-5.

Park, S. Y., J. H. Lee, et al. (2009). "miR-29 miRNAs activate p53 by targeting p85 alpha and CDC42." Nat Struct Mol Biol 16(1): 23-9.

Pavletich, N. P., K. A. Chambers, et al. (1993). "The DNA-binding domain of p53 contains the four conserved regions and the major mutation hot spots." Genes Dev 7(12B): 2556-64.

Pereg, Y., D. Shkedy, et al. (2005). "Phosphorylation of Hdmx mediates its Hdm2- and ATM-dependent degradation in response to DNA damage." Proc Natl Acad Sci U S A 102(14): 5056-61.

Petersen, C. P., M. E. Bordeleau, et al. (2006). "Short RNAs repress translation after initiation in mammalian cells." Mol Cell 21(4): 533-42.

Pillai, R. S., S. N. Bhattacharyya, et al. (2005). "Inhibition of translational initiation by Let-7 MicroRNA in human cells." Science 309(5740): 1573-6.

Place, R. F., L. C. Li, et al. (2008). "MicroRNA-373 induces expression of genes with complementary promoter sequences." Proc Natl Acad Sci U S A 105(5): 1608-13.

Poliseno, L., L. Salmena, et al. (2010). "Identification of the miR-106b 25 microRNA cluster as a proto-oncogenic PTEN-targeting intron that cooperates with its host gene MCM7 in transformation." Sci Signal 3(117): ra29. 
Prives, C. and V. Gottifredi (2008). "The p21 and PCNA partnership: a new twist for an old plot." Cell Cycle 7(24): 3840-6.

Raver-Shapira, N., E. Marciano, et al. (2007). "Transcriptional activation of miR-34a contributes to p53-mediated apoptosis." Mol Cell 26(5): 731-43.

Raver-Shapira, N. and M. Oren (2007). "Tiny actors, great roles: microRNAs in p53's service." Cell Cycle 6(21): 2656-61.

Rinaldo, C., A. Prodosmo, et al. (2007). "MDM2-regulated degradation of HIPK2 prevents p53Ser46 phosphorylation and DNA damage-induced apoptosis." Mol Cell 25(5): 739-50.

Rodriguez, A., S. Griffiths-Jones, et al. (2004). "Identification of mammalian microRNA host genes and transcription units." Genome Res 14(10A): 1902-10.

Ruby, J. G., C. H. Jan, et al. (2007). "Intronic microRNA precursors that bypass Drosha processing." Nature 448(7149): 83-6.

Rybak, A., H. Fuchs, et al. (2008). "A feedback loop comprising lin-28 and let-7 controls pre-let-7 maturation during neural stem-cell commitment." Nat Cell Biol 10(8): 987-93.

Sachdeva, M., S. Zhu, et al. (2009). "p53 represses c-Myc through induction of the tumor suppressor miR-145." Proc Natl Acad Sci U S A 106(9): 3207-12.

Sakaguchi, K., S. Saito, et al. (2000). "Damage-mediated phosphorylation of human p53 threonine 18 through a cascade mediated by a casein 1-like kinase. Effect on Mdm2 binding." J Biol Chem 275(13): 9278-83.

Sampson, V. B., N. H. Rong, et al. (2007). "MicroRNA let-7a down-regulates MYC and reverts MYC-induced growth in Burkitt lymphoma cells." Cancer Res 67(20): 9762-70.

Samuels-Lev, Y., D. J. O'Connor, et al. (2001). "ASPP proteins specifically stimulate the apoptotic function of p53." Mol Cell 8(4): 781-94.

Sansam, C. L., J. L. Shepard, et al. (2006). "DTL/CDT2 is essential for both CDT1 regulation and the early G2/M checkpoint." Genes Dev 20(22): 3117-29.

Sanvito, F., S. Piatti, et al. (1999). "The beta4 integrin interactor p27(BBP/elF6) is an essential nuclear matrix protein involved in $60 \mathrm{~S}$ ribosomal subunit assembly." $\mathrm{J}$ Cell Biol 144(5): 823-37.

Schetter, A. J., S. Y. Leung, et al. (2008). "MicroRNA expression profiles associated with prognosis and therapeutic outcome in colon adenocarcinoma." JAMA 299(4): 425-36.

Schwarz, D. S., G. Hutvagner, et al. (2003). "Asymmetry in the assembly of the RNAi enzyme complex." Cell 115(2): 199-208.

Segev, D. L., M. Saji, et al. (1998). "Polymerase chain reaction-based microsatellite polymorphism analysis of follicular and Hurthle cell neoplasms of the thyroid." $\mathrm{J}$ Clin Endocrinol Metab 83(6): 2036-42.

Seggerson, K., L. Tang, et al. (2002). "Two genetic circuits repress the Caenorhabditis elegans heterochronic gene lin-28 after translation initiation." Dev Biol 243(2): 215-25.

Serrano, M., A. W. Lin, et al. (1997). "Oncogenic ras provokes premature cell senescence associated with accumulation of p53 and p16INK4a." Cell 88(5): 593-602. Sharma, N., C. Timmers, et al. (2006). "Control of the p53-p21CIP1 Axis by E2f1, E2f2, and E2f3 is essential for G1/S progression and cellular transformation." J Biol Chem 281(47): 36124-31. 
$\mathrm{Si}, \mathrm{K}$. and U. Maitra (1999). "The Saccharomyces cerevisiae homologue of mammalian translation initiation factor 6 does not function as a translation initiation factor." $\underline{\text { Mol Cell }}$ Biol 19(2): 1416-26.

Song, B., Y. Wang, et al. (2008). "miR-192 Regulates dihydrofolate reductase and cellular proliferation through the p53-microRNA circuit." Clin Cancer Res 14(24): 8080-6.

Song, B., Y. Wang, et al. (2010). "Molecular mechanism of chemoresistance by miR-215 in osteosarcoma and colon cancer cells." Mol Cancer 9(1): 96.

Song, G. and L. Wang (2008). "MiR-433 and miR-127 arise from independent overlapping primary transcripts encoded by the miR-433-127 locus." PLoS One 3(10): e3574.

Sparmann, A. and M. van Lohuizen (2006). "Polycomb silencers control cell fate, development and cancer." Nat Rev Cancer 6(11): 846-56.

Stommel, J. M. and G. M. Wahl (2004). "Accelerated MDM2 auto-degradation induced by DNA-damage kinases is required for p53 activation." EMBO J 23(7): 1547-56.

Su, H., J. R. Yang, et al. (2009). "MicroRNA-101, down-regulated in hepatocellular carcinoma, promotes apoptosis and suppresses tumorigenicity." Cancer Res 69(3): 1135-42.

Sugimoto, M., A. Gromley, et al. (2006). "Hzf, a p53-responsive gene, regulates maintenance of the G2 phase checkpoint induced by DNA damage." Mol Cell Biol 26(2): 502-12.

Sugiyama, T., H. Cam, et al. (2005). "RNA-dependent RNA polymerase is an essential component of a self-enforcing loop coupling heterochromatin assembly to siRNA production." Proc Natl Acad Sci U S A 102(1): 152-7.

Sun, M., Z. Estrov, et al. (2008). "Curcumin (diferuloylmethane) alters the expression profiles of microRNAs in human pancreatic cancer cells." Mol Cancer Ther 7(3): 464-73.

Sykes, S. M., H. S. Mellert, et al. (2006). "Acetylation of the p53 DNA-binding domain regulates apoptosis induction." Mol Cell 24(6): 841-51.

Takamizawa, J., H. Konishi, et al. (2004). "Reduced expression of the let-7 microRNAs in human lung cancers in association with shortened postoperative survival." Cancer Res 64(11): 3753-6.

Tang, Y., J. Luo, et al. (2006). "Tip60-dependent acetylation of p53 modulates the decision between cell-cycle arrest and apoptosis." Mol Cell 24(6): 827-39.

Tang, Y., W. Zhao, et al. (2008). "Acetylation is indispensable for p53 activation." Cell 133(4): 612-26.

Tarasov, V., P. Jung, et al. (2007). "Differential regulation of microRNAs by p53 revealed by massively parallel sequencing: miR-34a is a p53 target that induces apoptosis and G1-arrest." Cell Cycle 6(13): 1586-93.

Tazawa, H., N. Tsuchiya, et al. (2007). "Tumor-suppressive miR-34a induces senescence-like growth arrest through modulation of the E2F pathway in human colon cancer cells." Proc Natl Acad Sci U S A 104(39): 15472-7.

Thomson, J. M., M. Newman, et al. (2006). "Extensive post-transcriptional regulation of microRNAs and its implications for cancer." Genes Dev 20(16): 2202-7. 
Timmers, C., N. Sharma, et al. (2007). "E2f1, E2f2, and E2f3 control E2F target expression and cellular proliferation via a p53-dependent negative feedback loop." Mol Cell Biol 27(1): 65-78.

Ting, Y., D. J. Medina, et al. (2010). "Differentiation-associated miR-22 represses Max expression and inhibits cell cycle progression." Biochem Biophys Res Commun 394(3): 606-11.

Tomari, Y., T. Du, et al. (2007). "Sorting of Drosophila small silencing RNAs." Cell 130(2): 299-308.

Uldrijan, S., W. J. Pannekoek, et al. (2007). "An essential function of the extreme C-terminus of MDM2 can be provided by MDMX." EMBO J 26(1): 102-12.

Varambally, S., Q. Cao, et al. (2008). "Genomic loss of microRNA-101 leads to overexpression of histone methyltransferase EZH2 in cancer." Science 322(5908): 1695-9.

Vassilev, L. T., B. T. Vu, et al. (2004). "In vivo activation of the p53 pathway by small-molecule antagonists of MDM2." Science 303(5659): 844-8.

Vasudevan, S. and J. A. Steitz (2007). "AU-rich-element-mediated upregulation of translation by FXR1 and Argonaute 2." Cell 128(6): 1105-18.

Vasudevan, S., Y. Tong, et al. (2007). "Switching from repression to activation: microRNAs can up-regulate translation." Science 318(5858): 1931-4.

Vaziri, H., S. K. Dessain, et al. (2001). "hSIR2(SIRT1) functions as an NAD-dependent p53 deacetylase." Cell 107(2): 149-59.

Venot, C., M. Maratrat, et al. (1998). "The requirement for the p53 proline-rich functional domain for mediation of apoptosis is correlated with specific PIG3 gene transactivation and with transcriptional repression." EMBO J 17(16): 4668-79.

Verdel, A., S. Jia, et al. (2004). "RNAi-mediated targeting of heterochromatin by the RITS complex." Science 303(5658): 672-6.

Volinia, S., G. A. Calin, et al. (2006). "A microRNA expression signature of human solid tumors defines cancer gene targets." Proc Natl Acad Sci U S A 103(7): 2257-61.

Voorhoeve, P. M., C. le Sage, et al. (2006). "A genetic screen implicates miRNA-372 and miRNA-373 as oncogenes in testicular germ cell tumors." Cell 124(6): 1169-81.

Vousden, K. H. and C. Prives (2009). "Blinded by the Light: The Growing Complexity of p53." Cell 137(3): 413-31.

Ward, L. S., G. Brenta, et al. (1998). "Studies of allelic loss in thyroid tumors reveal major differences in chromosomal instability between papillary and follicular carcinomas." J Clin Endocrinol Metab 83(2): 525-30.

Weber, J. D., L. J. Taylor, et al. (1999). "Nucleolar Arf sequesters Mdm2 and activates p53." Nat Cell Biol 1(1): 20-6.

Weinmann, L., J. Hock, et al. (2009). "Importin 8 is a gene silencing factor that targets argonaute proteins to distinct mRNAs." Cell 136(3): 496-507.

Welch, C., Y. Chen, et al. (2007). "MicroRNA-34a functions as a potential tumor suppressor by inducing apoptosis in neuroblastoma cells." Oncogene 26(34): 5017-22. Winter, J., S. Jung, et al. (2009). "Many roads to maturity: microRNA biogenesis pathways and their regulation." Nat Cell Biol 11(3): 228-34. 
Wu, L. and J. G. Belasco (2005). "Micro-RNA regulation of the mammalian lin-28 gene during neuronal differentiation of embryonal carcinoma cells." Mol Cell Biol 25(21): 9198-208.

Wu, L., C. Timmers, et al. (2001). "The E2F1-3 transcription factors are essential for cellular proliferation." Nature 414(6862): 457-62.

Wulczyn, F. G., L. Smirnova, et al. (2007). "Post-transcriptional regulation of the let-7 microRNA during neural cell specification." FASEB J 21(2): 415-26.

Xiong, J., D. Yu, et al. (2010). "An estrogen receptor alpha suppressor, microRNA-22, is downregulated in estrogen receptor alpha-positive human breast cancer cell lines and clinical samples." FEBS J 277(7): 1684-94.

Xirodimas, D. P., M. K. Saville, et al. (2004). "Mdm2-mediated NEDD8 conjugation of p53 inhibits its transcriptional activity." Cell 118(1): 83-97.

Xue, W., L. Zender, et al. (2007). "Senescence and tumour clearance is triggered by p53 restoration in murine liver carcinomas." Nature 445(7128): 656-60.

Yamakuchi, M., M. Ferlito, et al. (2008). "miR-34a repression of SIRT1 regulates apoptosis." Proc Natl Acad Sci U S A 105(36): 13421-6.

Yanaihara, N., N. Caplen, et al. (2006). "Unique microRNA molecular profiles in lung cancer diagnosis and prognosis." Cancer Cell 9(3): 189-98.

Yi, R., Y. Qin, et al. (2003). "Exportin-5 mediates the nuclear export of pre-microRNAs and short hairpin RNAs." Genes Dev 17(24): 3011-6.

Yu, J., Z. Wang, et al. (2003). "PUMA mediates the apoptotic response to p53 in colorectal cancer cells." Proc Natl Acad Sci U S A 100(4): 1931-6.

$\mathrm{Yu}$, J. and L. Zhang (2003). "No PUMA, no death: implications for p53-dependent apoptosis." Cancer Cell 4(4): 248-9.

Zedenius, J., G. Wallin, et al. (1995). "Allelotyping of follicular thyroid tumors." Genet 96(1): 27-32.

Zeng, Y., X. Cai, et al. (2005). "Use of RNA polymerase II to transcribe artificial microRNAs." Methods Enzymol 392: 371-80.

Zeng, Y. and B. R. Cullen (2004). "Structural requirements for pre-microRNA binding and nuclear export by Exportin 5." Nucleic Acids Res 32(16): 4776-85.

Zhan, Q., M. J. Antinore, et al. (1999). "Association with Cdc2 and inhibition of Cdc2/Cyclin B1 kinase activity by the p53-regulated protein Gadd45." Oncogene 18(18): 2892-900. 


\section{Abbreviations}

\begin{tabular}{|c|c|}
\hline Ago & Argonaute \\
\hline ASPP & Apoptosis-stimulating protein of $\mathrm{p} 53$ \\
\hline BSA & Bovine serum albumin \\
\hline $\mathrm{CBP}$ & CREB-binding protein \\
\hline ChIP & Chromatin immunoprecipitation \\
\hline CLL & Chronic lymphocytic leukemia \\
\hline $\mathrm{CPT}$ & Camptothecin \\
\hline DBD & DNA-binding domain \\
\hline DGCR8 & DiGeorge syndrome critical region gene 8 \\
\hline DMSO & Dimethylsulphoxide \\
\hline DOX & Doxorubicin \\
\hline DSB & Double-strand break \\
\hline FACS & Fluorescence activated cell sorting analysis \\
\hline $\mathrm{HCC}$ & Hepatocellular carcinoma \\
\hline IP & Immunoprecipitation \\
\hline IRES & Internal ribosome entry site \\
\hline miRNA & MicroRNA \\
\hline MSI & Unstable microsatellite \\
\hline MSS & Microsatellite stable \\
\hline OMM & Outer mitochondrial membrane \\
\hline PcG & Polycomb group \\
\hline PCR & Polymerase chain reaction \\
\hline $\mathrm{RE}$ & Response element \\
\hline Pri-miRNA & Primary miRNA transcript \\
\hline PRC & Polycomb repressive complex \\
\hline RLC & RISC loading complex \\
\hline RNAa & RNA activation \\
\hline
\end{tabular}


RNAi

RNA Pol II

RISC

RITS

RT-PCR

SDS

TET

TGF $\beta$

5-FU
RNA interference

RNA polymerase II

RNA-induced silencing complex

RNA-induced transcriptional site

Real-time PCR

Sodium dodecylsulfate

Tetramerization domain

Transforming growth factor- $\beta$

5-fluorouracil 


\section{Acknowledgements}

More than one month I spent on thesis writing. Now it is ending! I do not even know how to describe my feeling at this moment. Hard time, big pressure, but I really enjoyed it. I have to say goodbye to the library, where I stayed most of my time during the last month. Now it is time to come back to the lab to continue my work. I am happy to see all the familiar faces again.

Matthias, I would like to thank you first. Since I came to Europe almost seven years ago, I have been moved from Odense to Marburg, then to Goettingen. And your lab moved from Marburg to Odense, and then to Goettingen. That was the opposite way, but finally we met in Goettingen almost five years ago. On that interview day, I remembered only you, Antje and Cathrin in the lab. Now our department has three independent groups, more than 20 people. That is a really amazing change. Thank you for giving me the opportunity twice to work in your lab. You helped me a lot, not only on my work, but also in my personal life. You are always gentle and patient, making me comfortable working in this lab.

I would like to thank Prof. Pieler and Prof. Wienands, the members of my thesis committee, for giving me constructive suggestions on my project.

My work would be mission impossible without the help from my dear colleagues. From you, I learned a lot and also had lots of fun. This year many of you will graduate sooner or later like me. I wish you all the best and great success in the future. My special thanks go to Irina and Muriel for your understanding and sharing. I would also like to thank Christian, Andreas and Antje for our fantastic, productive collaboration.

I am really happy that I made so many friends here in Goettingen. You are my great treasure. No matter where I am in the future, those days we were together will be always in my memory.

Finally, I would like to thank my parents and Zhuli. I really have to apologize that I accompanied my parents for so few days in the past seven years. I know you are always there supporting me. Zhuli, thank you for accompanying me through these tough days. I did so few for you, but you gave so much to me. Love all of you. 